\title{
Ligand Bias of Scoring Functions in Structure-Based Virtual Screening (Supporting Information)
}

\section{Micael Jacobsson ${ }^{*}, 1,2$ and Anders Karlén ${ }^{1}$}

1) Department of Med. Chem., Faculty of Pharmacy, Uni. of Uppsala, Box 574, SE-751 23 Uppsala, Sweden,

2) Department of Chemistry, Biovitrum AB, SE-112 76 Stockholm, Sweden

* Corresponding author e-mail: micael.jacobsson@biovitrum.com, phone: +46 86972551

CHEMISTRY<smiles>c1ccc(-c2ccc(NCCN3CCOCC3)nn2)cc1</smiles>

A1_1<smiles>c1ccc(CC2CCN(CCNc3ccc(-c4ccccc4)nn3)CC2)cc1</smiles>

A1_10<smiles>O=C(CN1CCC(Cc2ccccc2)CC1)Nc1ccc(-c2ccccc2)nn1</smiles>

A1_11<smiles>O=C(CN1CCN(Cc2ccccc2)CC1)Nc1ccc(-c2ccccc2)nn1</smiles>

A1_12<smiles>O=C(CNc1ccc(-c2ccccc2)nn1)N1CCN(Cc2ccccc2)CC1</smiles>

A1_13<smiles>c1ccc(-c2ccc(NCCCCN3CCc4ccccc4C3)nn2)cc1</smiles>

A1_14<smiles>CN(CCCNc1ccc(-c2ccccc2)nn1)Cc1ccccc1</smiles>

TARGET SOURCE

AChE Contreras

AChE Contreras

AChE Contreras

AChE Contreras

AChE Contreras

AChE Contreras 
<smiles>CN(CCCCNc1ccc(-c2ccccc2)nn1)Cc1ccccc1</smiles>

A1_16<smiles>c1ccc(-c2ccc(NCCCCCN3CCc4ccccc4C3)nn2)cc1</smiles>

A1 17<smiles>c1ccc(CN2CCC(CNc3ccc(-c4ccccc4)nn3)CC2)cc1</smiles>

A1_18<smiles>O=C(CC1CCN(Cc2ccccc2)CC1)Nc1ccc(-c2ccccc2)nn1</smiles>

A1_19<smiles>Cc1cc(-c2ccccc2)nnc1NCCN1CCOCC1</smiles>

A1_2<smiles>Cc1cc(C)c(-c2ccc(NCCC3CCN(Cc4ccccc4)CC3)nn2)c(C)c1</smiles>

A1_20<smiles>CN(CCCCCNc1ccc(-c2ccccc2)nn1)Cc1ccccc1</smiles>

A1_21<smiles>c1ccc(CN2CCC(CCNc3nnc(-c4ccccc4)c4ccccc34)CC2)cc1</smiles>

AChE Contreras

AChE Contreras

AChE Contreras

AChE Contreras

AChE Contreras

AChE Contreras

AChE Contreras

AChE Contreras 
<smiles>CC(C)c1cc(-c2ccccc2)nnc1NCCC1CCN(Cc2ccccc2)CC1</smiles>

A1_23<smiles>Cc1cc(-c2ccccc2)nnc1NCCC1CCN(Cc2ccccc2)CC1</smiles>

A1_24<smiles>c1ccc(CN2CCC(CCNc3cccnn3)CC2)cc1</smiles>

A1_25<smiles>COc1ccc(NCCC2CCN(Cc3ccccc3)CC2)nn1</smiles>

A1_26<smiles>c1ccc(CN2CCC(CCOc3ccc(-c4ccccc4)nn3)CC2)cc1</smiles>

A1_27<smiles>c1ccc(CN2CCC(CCNc3ccc(-c4ccccc4)nn3)CC2)cc1</smiles>

A1_28<smiles>COc1ccccc1-c1ccc(NCCC2CCN(Cc3ccccc3)CC2)nn1</smiles>

A1_29<smiles>c1ccc(-c2ccc(NCCCN3CCOCC3)nn2)cc1</smiles>

A1_3<smiles>c1ccc(CN2CCC(CCNc3ccc(-c4cccs4)nn3)CC2)cc1</smiles>

AChE Contreras

AChE Contreras

AChE Contreras

AChE Contreras

AChE Contreras

AChE Contreras

AChE Contreras

AChE Contreras

AChE Contreras 


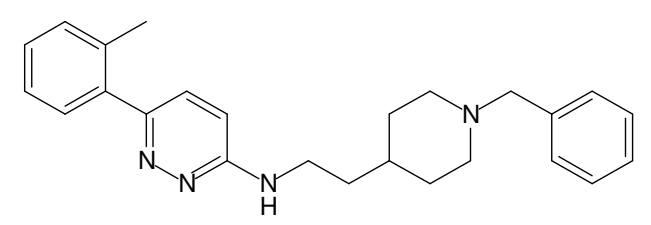

A1_31<smiles>CCc1ccccc1-c1ccc(NCCC2CCN(Cc3ccccc3)CC2)nn1</smiles>

A1_32<smiles>Clc1ccccc1-c1ccc(NCCC2CCN(Cc3ccccc3)CC2)nn1</smiles>

A1_33<smiles>Clc1ccc(NCCC2CCN(Cc3ccccc3)CC2)nn1</smiles>

A1_34

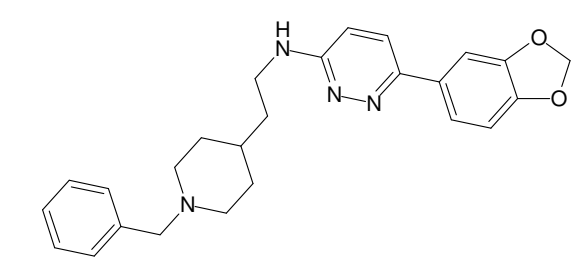

A1_35<smiles>c1ccc(CN2CCC(CCSc3ccc(-c4ccccc4)nn3)CC2)cc1</smiles>

A1_36<smiles>CCCc1cc(NCCC2CCN(Cc3ccccc3)CC2)nnc1-c1ccccc1</smiles>

A1_37<smiles>C1=C(c2ccc(NCCC3CCN(Cc4ccccc4)CC3)nn2)Cc2ccccc2C1</smiles>

A1_38<smiles>CC(=O)Nc1cccc(-c2ccc(NCCC3CCN(Cc4ccccc4)CC3)nn2)c1</smiles>

A1_39
AChE Contreras

AChE Contreras

AChE Contreras

AChE Contreras

AChE Contreras

AChE Contreras

AChE Contreras

AChE Contreras

AChE Contreras 
<smiles>CN(CCNc1ccc(-c2ccccc2)nn1)Cc1ccccc1</smiles>

A1 4<smiles>c1ccc(CN2CCC(CCNc3ccc(-c4ccccn4)nn3)CC2)cc1</smiles>

A1_40

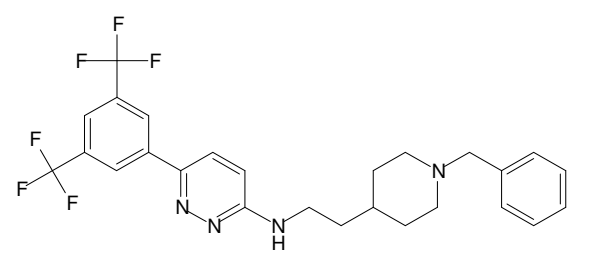

A1_41<smiles>CC(=O)c1cccc(-c2ccc(NCCC3CCN(Cc4ccccc4)CC3)nn2)c1</smiles>

A1_42<smiles>c1ccc(CN2CCC(CCNc3cc4c(nn3)-c3ccccc3CC4)CC2)cc1</smiles>

A1_43<smiles>CCc1cc(NCCC2CCN(Cc3ccccc3)CC2)nnc1-c1ccccc1</smiles>

A1_44<smiles>c1ccc(CN2CCC(CCNc3cc4ccc5ccccc5c4nn3)CC2)cc1</smiles>

A1_45

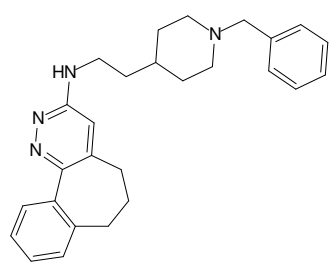

A1_46
AChE Contreras

AChE Contreras

AChE Contreras

AChE Contreras

AChE Contreras

AChE Contreras

AChE Contreras

AChE Contreras 


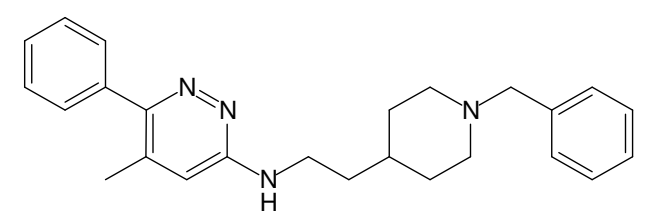

A1_47

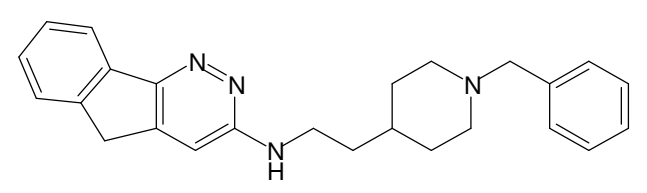

A1_48<smiles>c1ccc(CN2CCN(CCNc3ccc(-c4ccccc4)nn3)CC2)cc1</smiles>

A1_49<smiles>O=C(CNc1ccc(-c2ccccc2)nn1)N1CCC(Cc2ccccc2)CC1</smiles>

A1_5<smiles>N#Cc1ccc(-c2ccc(NCCC3CCN(Cc4ccccc4)CC3)nn2)cc1</smiles>

A1_50<smiles>Fc1ccc(-c2ccc(NCCC3CCN(Cc4ccccc4)CC3)nn2)cc1</smiles>

A1_51<smiles>CN(C)c1ccc(-c2ccc(NCCC3CCN(Cc4ccccc4)CC3)nn2)cc1</smiles>

A1_52<smiles>Nc1c2c(nc3ccccc13)CCCC2</smiles>

A1_53
AChE Contreras

AChE Contreras

AChE Contreras

AChE Contreras

AChE Contreras

AChE Contreras

AChE Contreras

AChE Contreras 


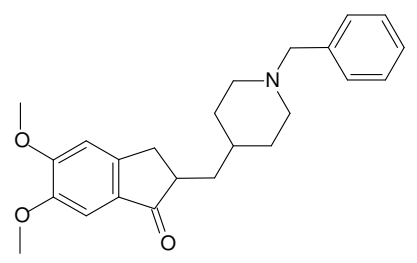

A1 54<smiles>c1ccc(-c2ccc(NCCN3CCc4ccccc4C3)nn2)cc1</smiles>

A1_6<smiles>c1ccc(-c2ccc(NCCN3CCCCC3)nn2)cc1</smiles>

A1_7<smiles>c1ccc(CN2CCC(Nc3ccc(-c4ccccc4)nn3)CC2)cc1</smiles>

A1_8

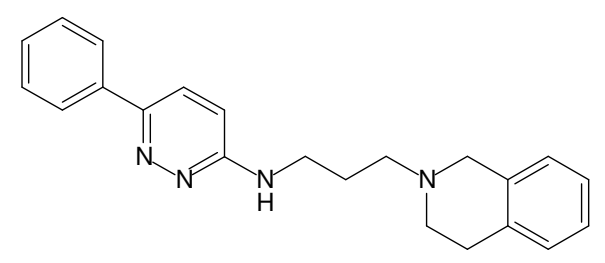

A1_9<smiles>CC[N+](C)(C)c1cccc(O)c1</smiles>

1AX9_lig

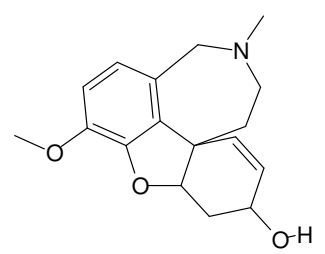

1DX6_lig

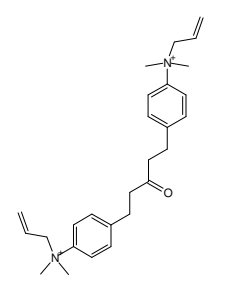

1E3Q_lig
AChE Contreras

AChE Contreras

AChE Contreras

AChE Contreras

AChE Contreras

AChE PDB

AChE PDB

AChE PDB 
1E66_lig
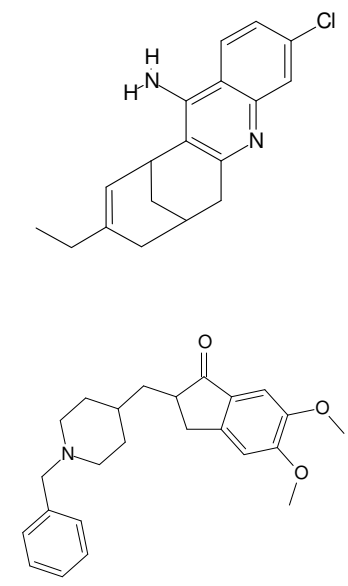

1EVE_lig

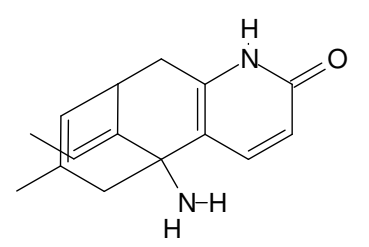

1GPK_lig

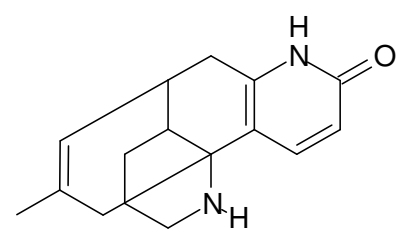

1GPN_lig

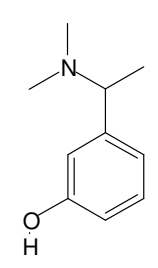

1GQS_lig

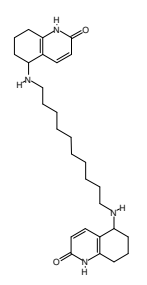

1H22_lig

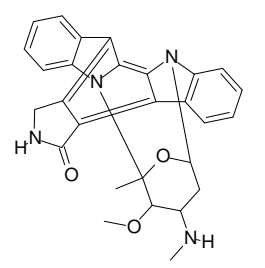

1aq1_lig

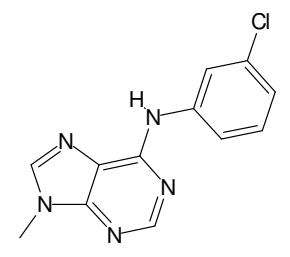

1ckp_lig
AChE PDB

AChE PDB

AChE PDB

AChE PDB

AChE PDB

AChE PDB

CDK2 PDB

CDK2 PDB 


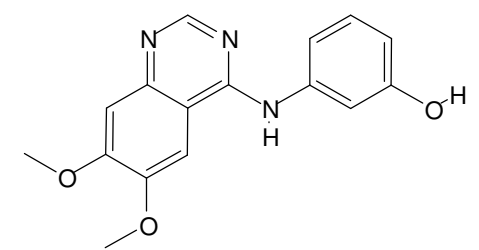

1di8_lig

CDK2 PDB

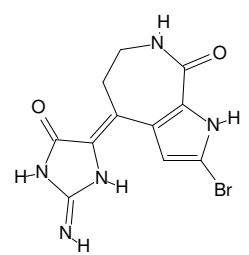

1dm2_lig

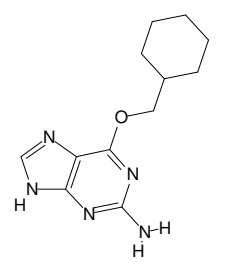

1e1v_lig

CDK2 PDB

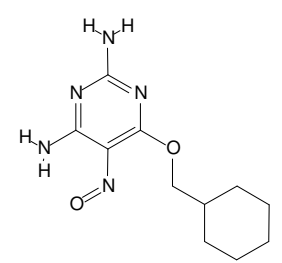

1e1x_lig

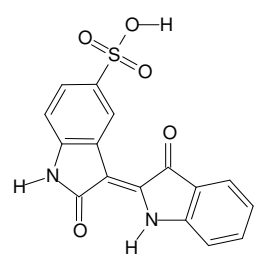

1e9h_lig

CDK2 PDB

CDK2 PDB

CDK2 PDB

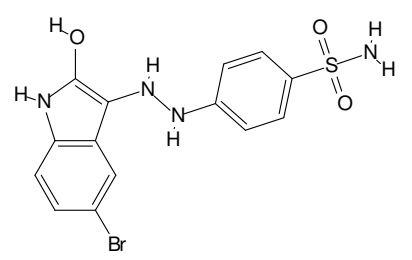

1fvt_lig

CDK2 PDB

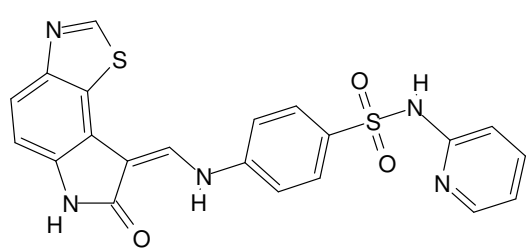

1fvv_lig

CDK2 PDB

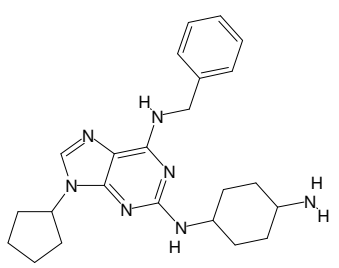

1g5s_lig

CDK2 PDB 


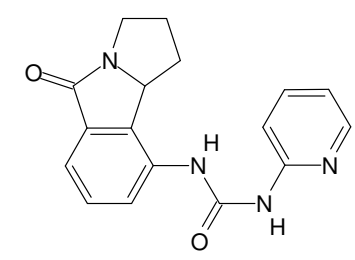

1gih_lig

CDK2 PDB

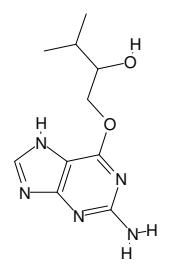

1gz8_lig

CDK2 PDB

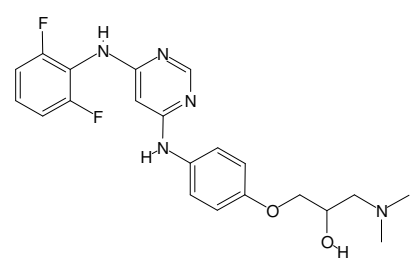

1h00_lig

CDK2 PDB

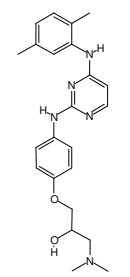

1h01_lig

CDK2 PDB

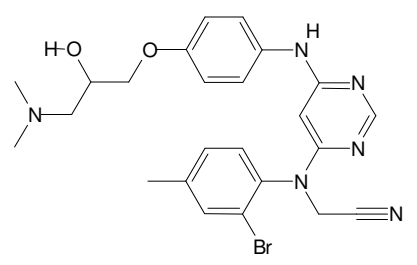

1h07_lig

CDK2 PDB

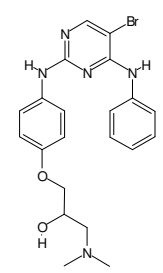

1h08_lig

CDK2 PDB

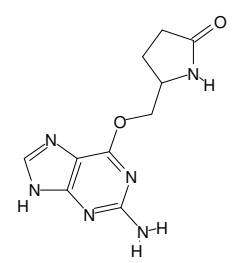

1hOv_lig

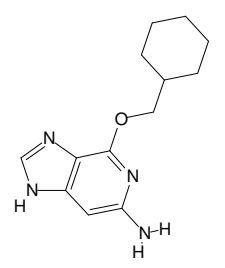

CDK2 PDB 


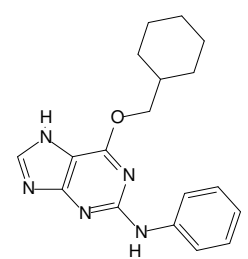

1h1q_lig

CDK2 PDB

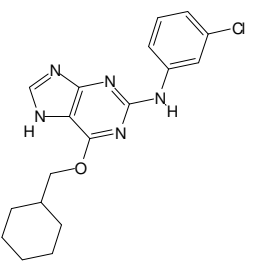

1h1r_lig

CDK2 PDB

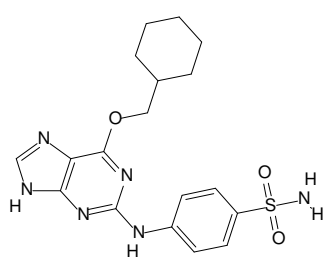

1h1s_lig

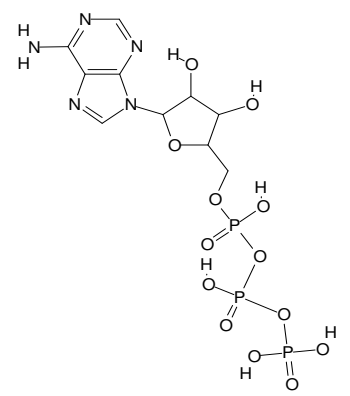

CDK2 PDB

1hck_lig

CDK2 PDB

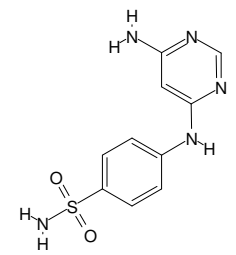

1jsv_lig

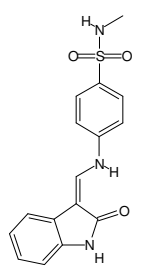

1ke5_lig

CDK2 PDB

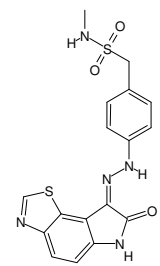

1ke6_lig

CDK2 PDB 


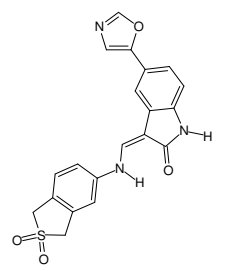

1ke7_lig

CDK2 PDB

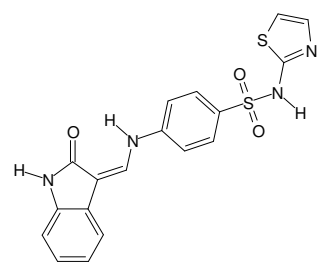

1ke8_lig

CDK2 PDB

1ke9_lig

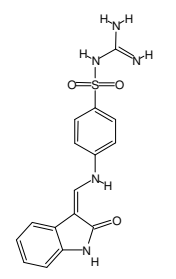

CDK2 PDB

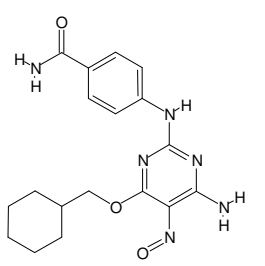

1ogu_lig

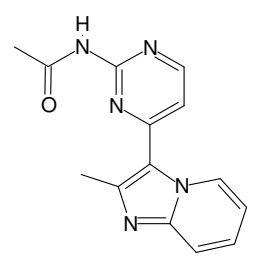

1oiq_lig

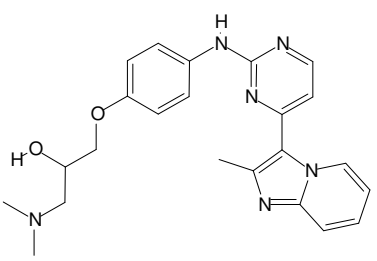

1oir_lig

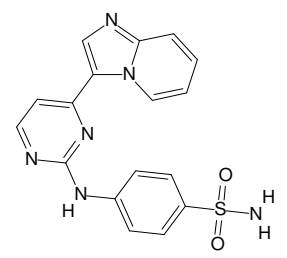

1oit_lig

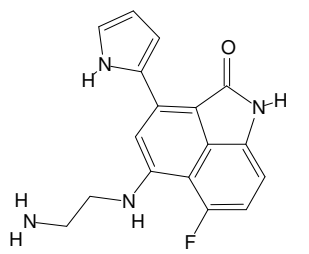

1p2a_lig

CDK2 PDB 


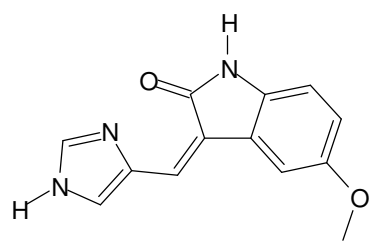

1pf8_lig

CDK2 PDB

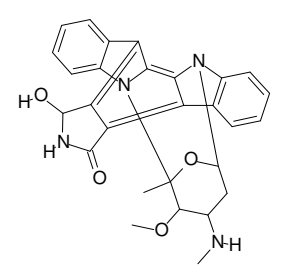

1pkd_lig

CDK2 PDB

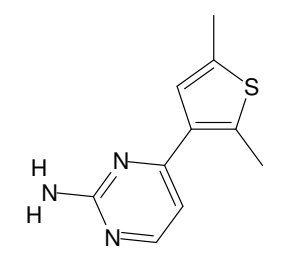

1pxi_lig

CDK2 PDB

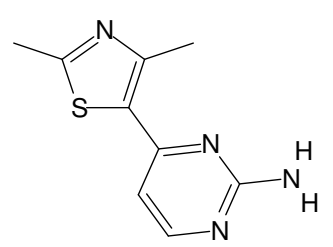

1pxj_lig

CDK2 PDB

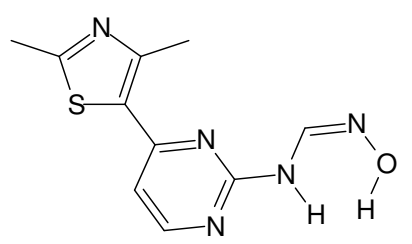

1pxk_lig

CDK2 PDB

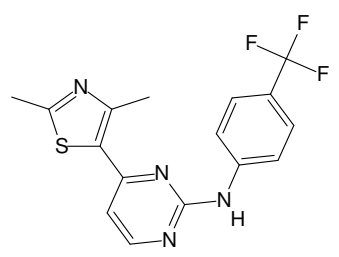

1pxl_lig

CDK2 PDB

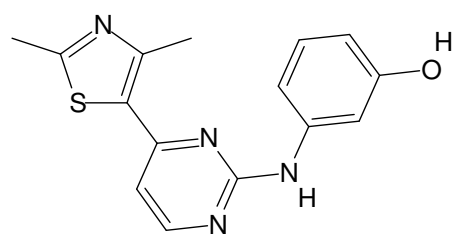

1pxm_lig

CDK2 PDB<smiles>CNc1nc(C)c(-c2ccnc(Nc3ccc(O)cc3)n2)s1</smiles>

1pxn_lig

CDK2 PDB 


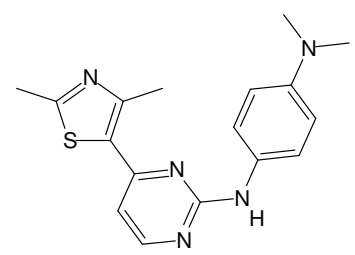

1pxp_lig

CDK2 PDB

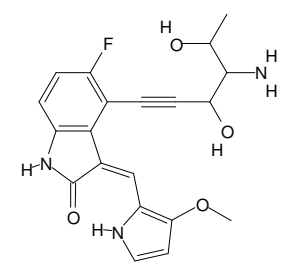

1r78_lig

CDK2 PDB

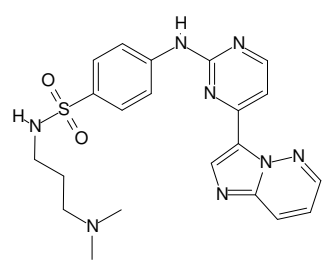

1urw_lig

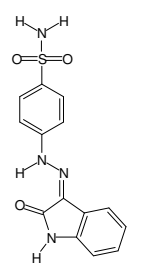

c1

CDK2 Tominaga

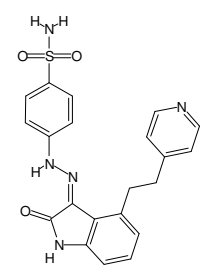

c10

CDK2 Tominaga

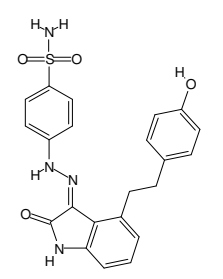

c11

CDK2 Tominaga

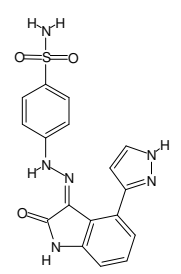

c12

CDK2 Tominaga

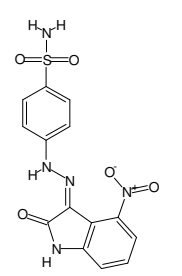




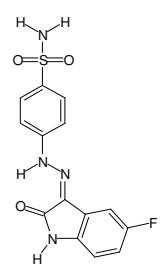

c15

CDK2 Tominaga

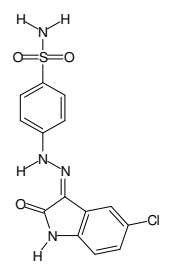

c16

CDK2 Tominaga

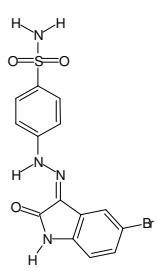

c17

CDK2 Tominaga

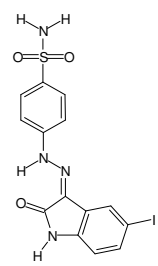

c18

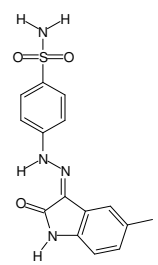

c19

\section{CDK2 Tominaga}

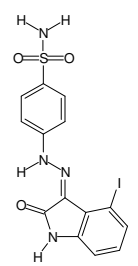

c2

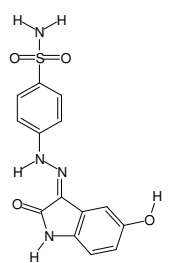

c20

CDK2 Tominaga

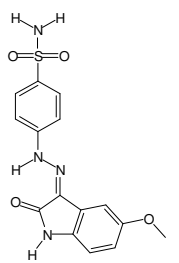

CDK2 Tominaga 


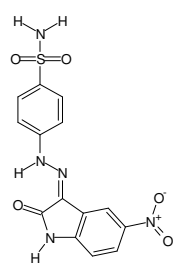

c22

CDK2 Tominaga

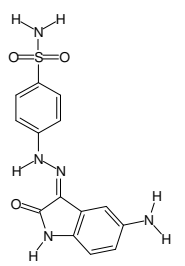

c23

CDK2 Tominaga

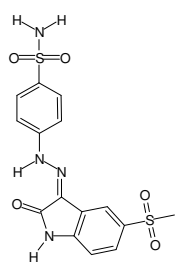

c24

CDK2 Tominaga

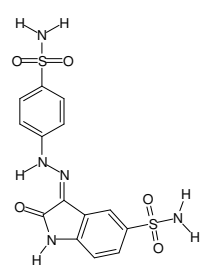

c25

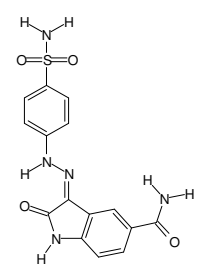

CDK2 Tominaga

c26

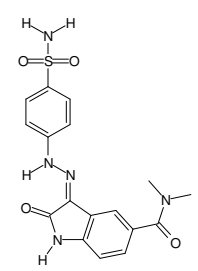

CDK2 Tominaga

c27

CDK2 Tominaga

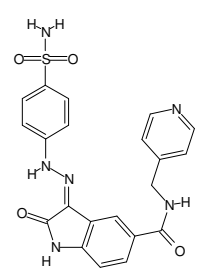

c28

CDK2 Tominaga

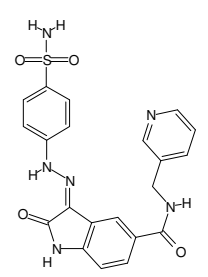




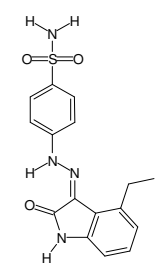

c3

CDK2 Tominaga

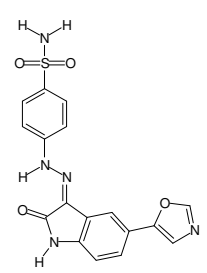

c30

CDK2 Tominaga

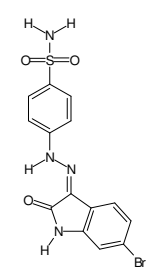

c31

CDK2 Tominaga

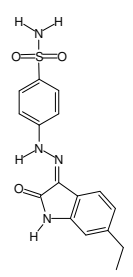

c32

CDK2 Tominaga

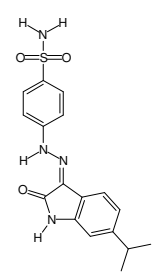

c33

CDK2 Tominaga

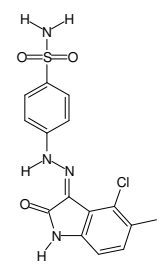

c35

CDK2 Tominaga

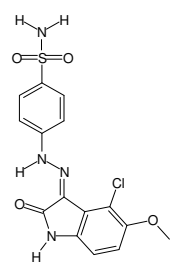

c36

CDK2 Tominaga

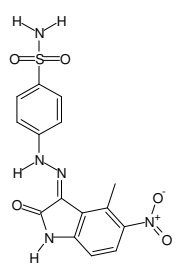




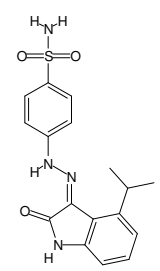

c4

CDK2 Tominaga

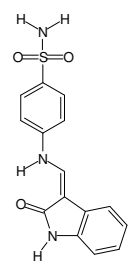

c41

CDK2 Tominaga

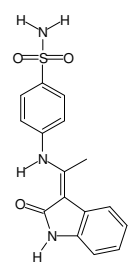

c42

CDK2 Tominaga

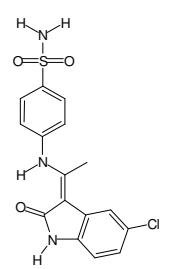

c43

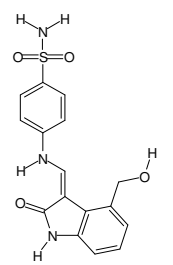

CDK2 Tominaga

CDK2 Tominaga

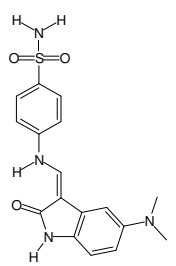

c45

CDK2 Tominaga

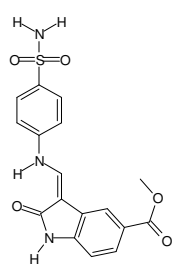

c46

CDK2 Tominaga

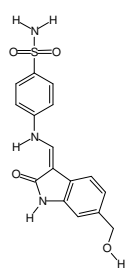




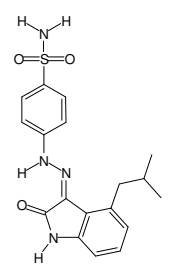

c5

CDK2 Tominaga

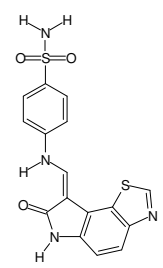

c50

CDK2 Tominaga

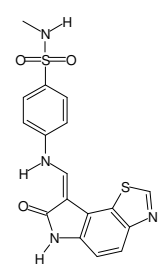

c51

CDK2 Tominaga

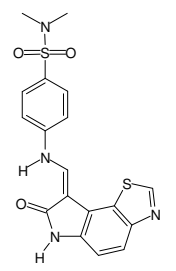

c52

CDK2 Tominaga

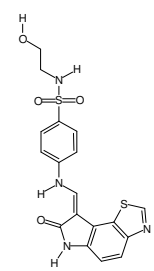

c53

CDK2 Tominaga

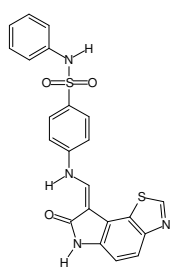

c54

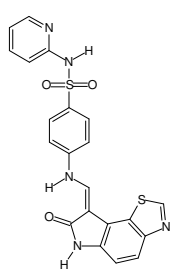

CDK2 Tominaga

c55

CDK2 Tominaga

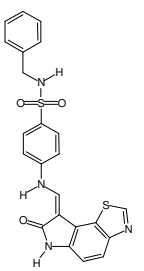




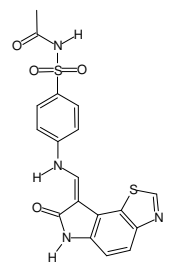

c57

CDK2 Tominaga

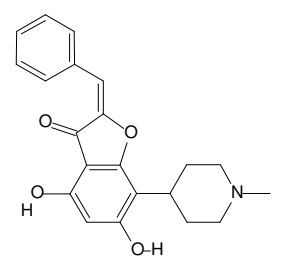

c58

CDK2 Tominaga

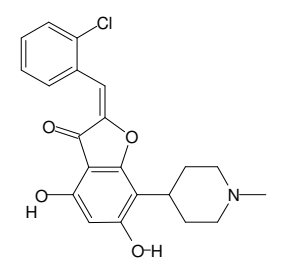

CDK2 Tominaga

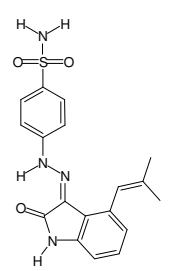

c6

CDK2 Tominaga

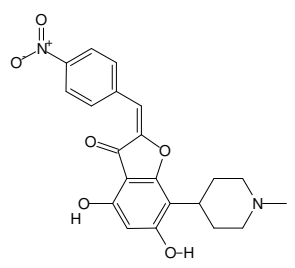

c60

CDK2 Tominaga

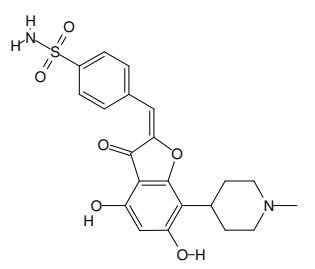

c61

CDK2 Tominaga

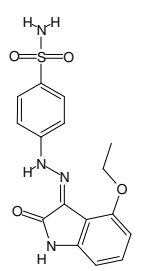

c7

CDK2 Tominaga

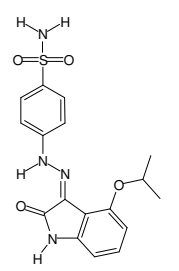

c8 


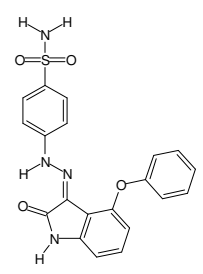

c9

CDK2 Tominaga

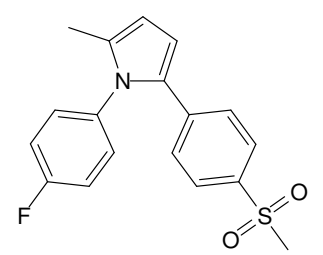

X001_A

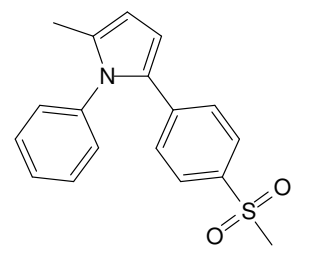

X002_A

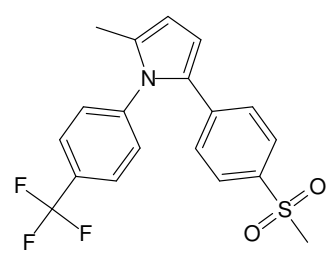

cox2 Clark

X003_A

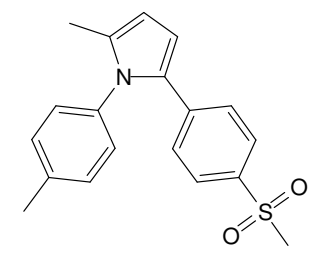

X004_A

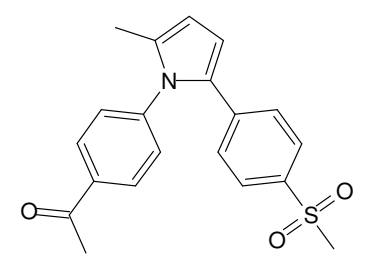

X005_A

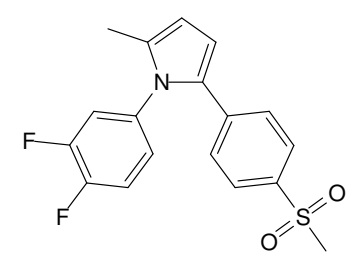

X006 A

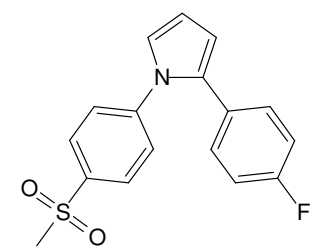




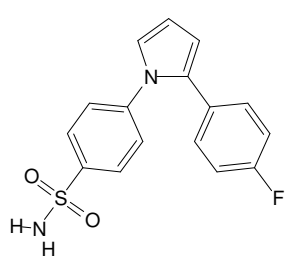

X008_A

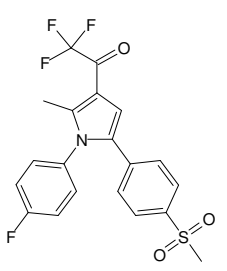

X009_A

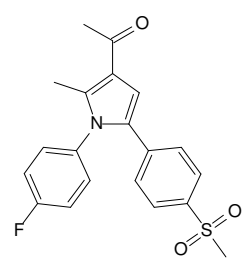

X010_A

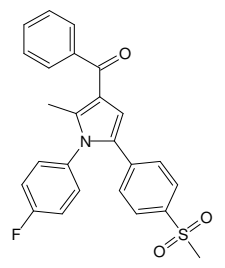

X011_A

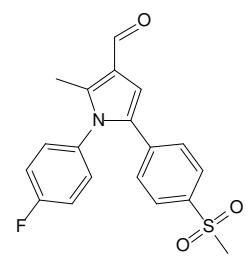

X012 A

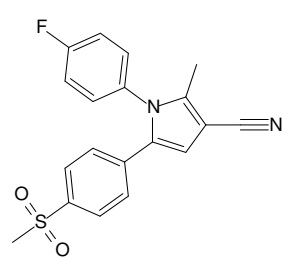

COX2 Clark

cox2 Clark

cox2 Clark

cox2 Clark

cox2 Clark

X013_A

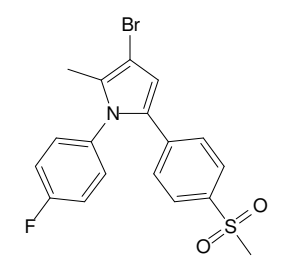

X014_A

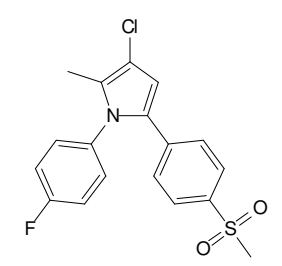

X015_A

cox2 Clark 


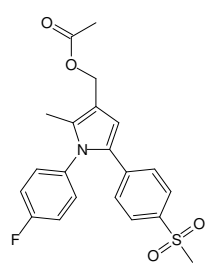

X016_A

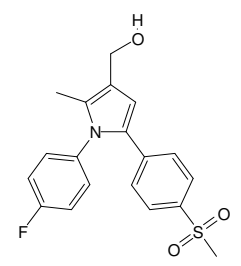

X017_A

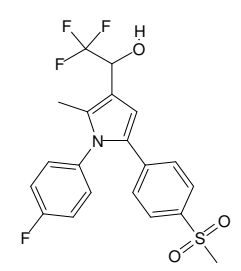

X018_AR

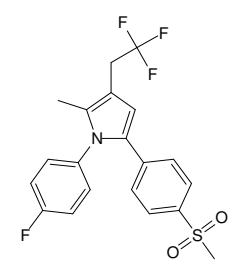

X019 A

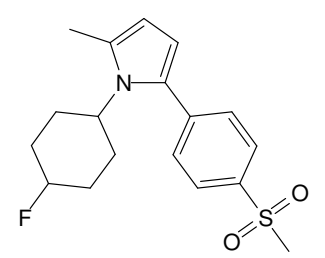

COX2 Clark

X020_A

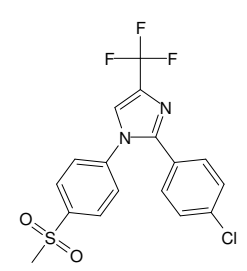

X021

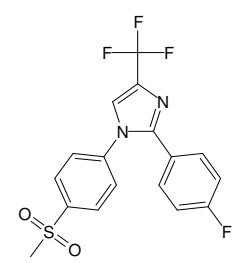

X022

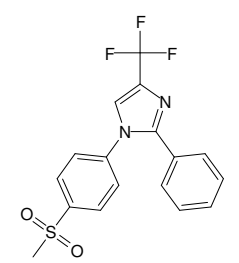




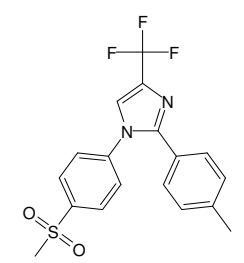

X024

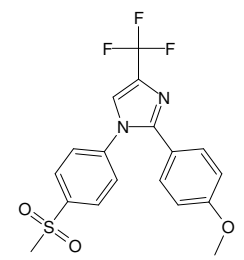

X025

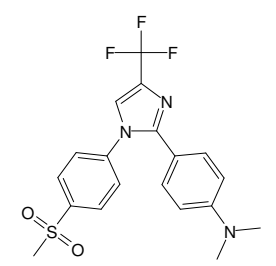

X026

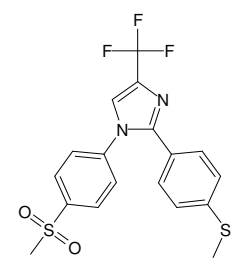

X027

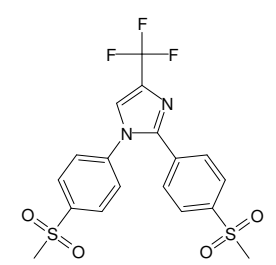

X028

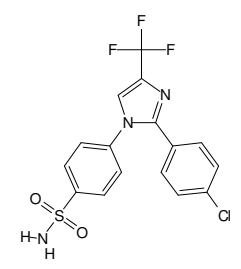

X029

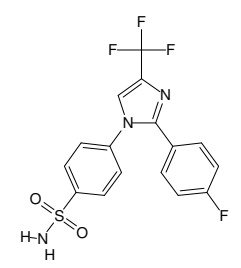

X030

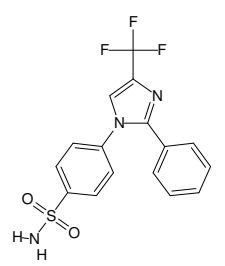

cox2 Clark

cox2 Clark

cox2 Clark

cox2 Clark

cox2 Clark

cox2 Clark

cox2 Clark COX2 Clark 


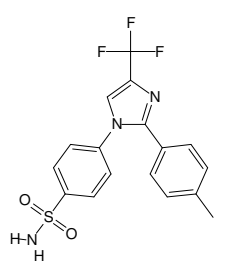

X032

cox2 Clark

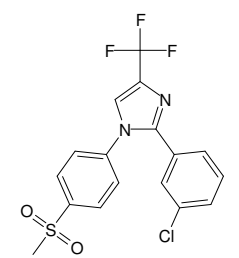

X033

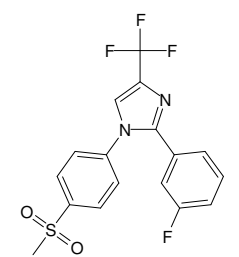

X034

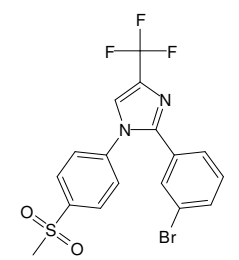

X035

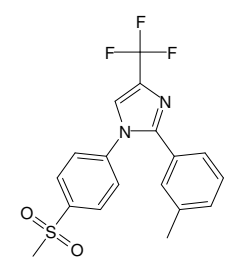

X036

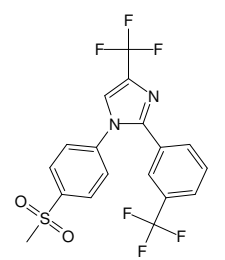

X037

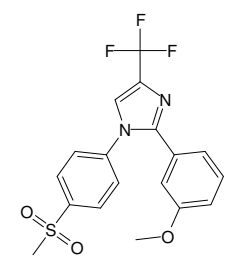

X038

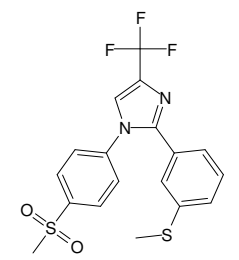




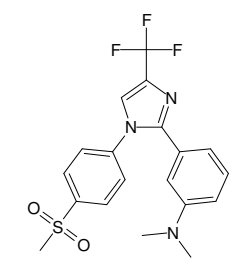

X040

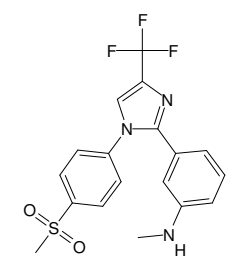

X041

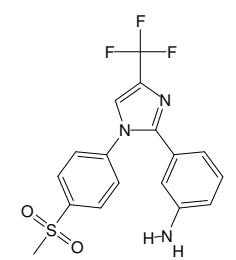

X042

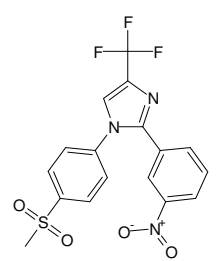

X043

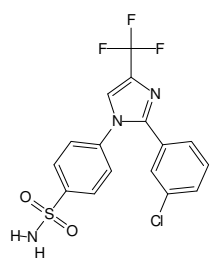

X044

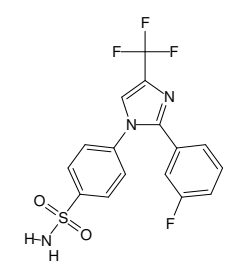

X045

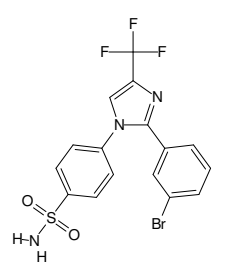

X046

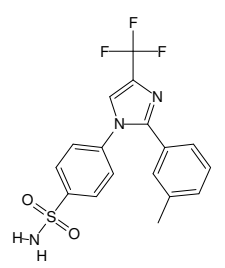




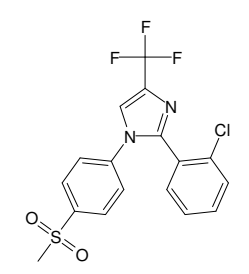

X048

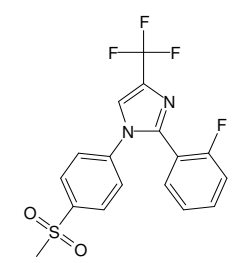

X049

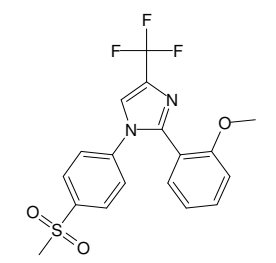

X050

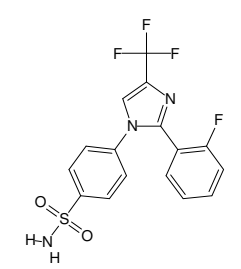

X051

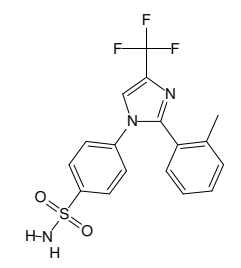

X052

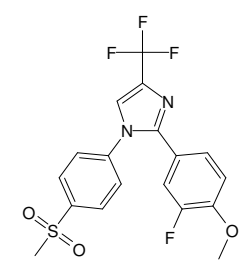

X053

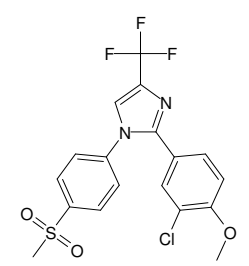

X054

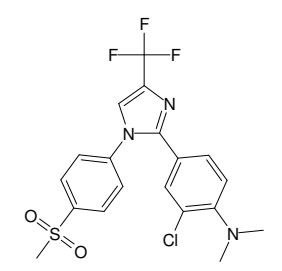




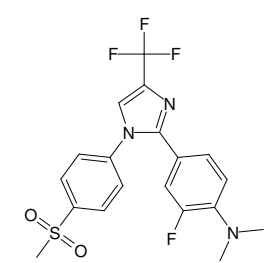

X056

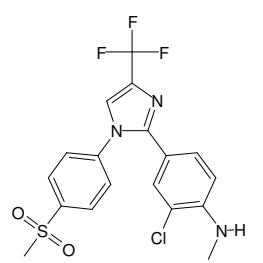

X057

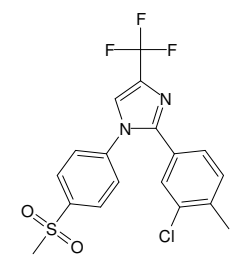

X058

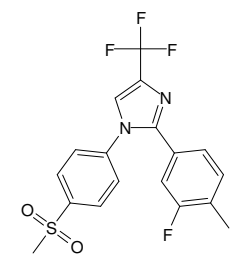

X059

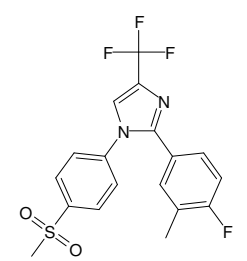

X060

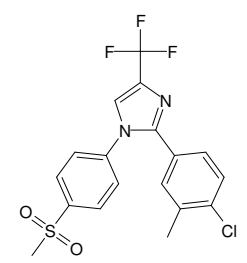

X061

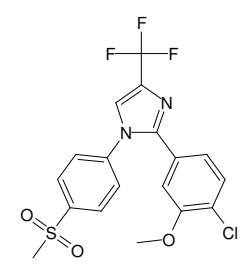

X062

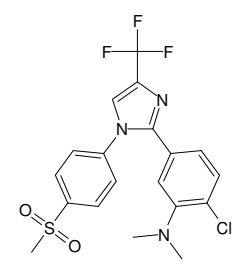

cox2 Clark

cox2 Clark

cox2 Clark

cox2 Clark

cox2 Clark

cox2 Clark

cox2 Clark cox2 Clark 


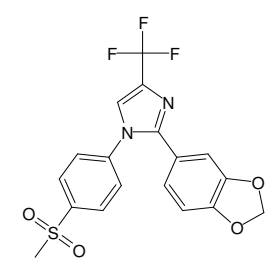

X064

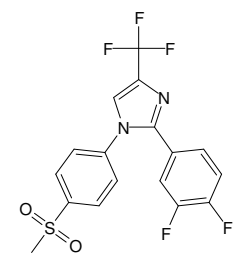

X065

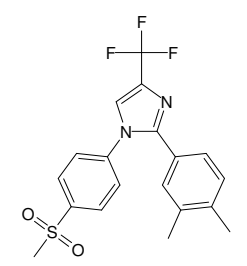

X066

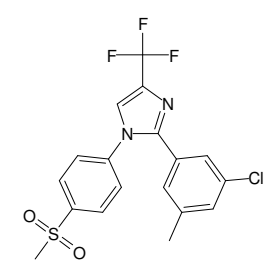

X067

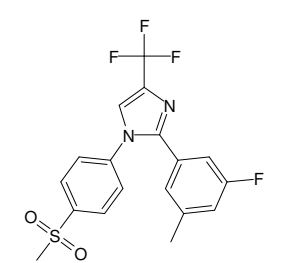

X068

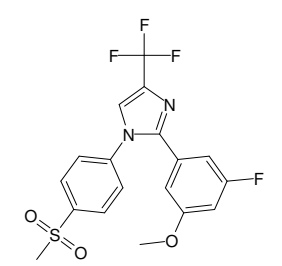

X069

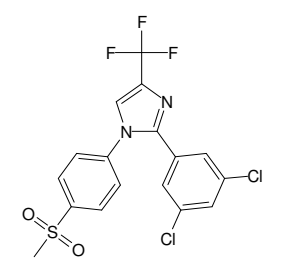

X070

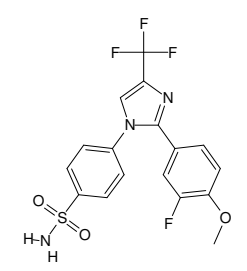




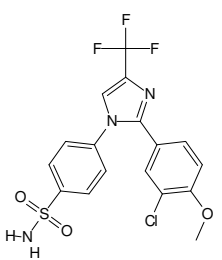

X072

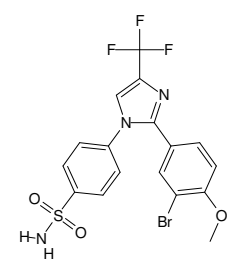

X073

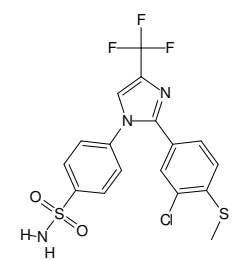

X074

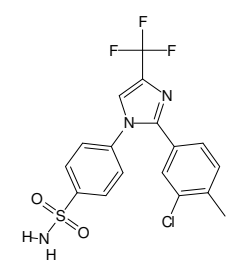

X075

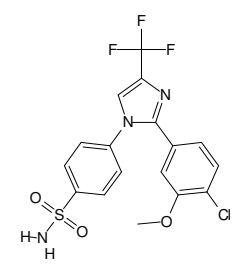

X076

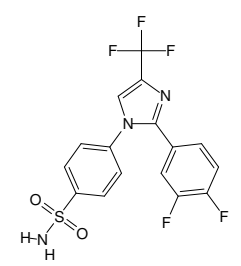

X077

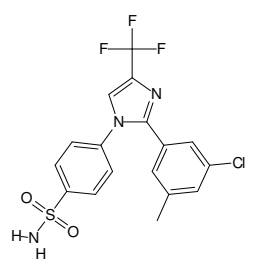

X078

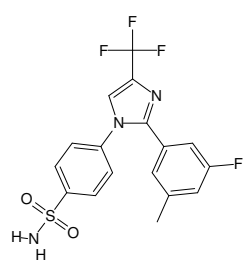

cox2 Clark

cox2 Clark

cox2 Clark

cox2 Clark

COX2 Clark

cox2 Clark

cox2 Clark COX2 Clark 


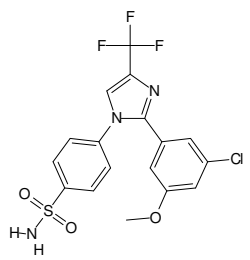

X080

cox2 Clark

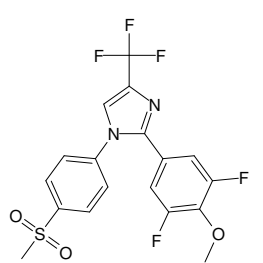

X081

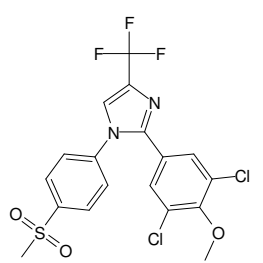

X082

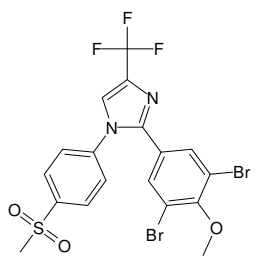

X083

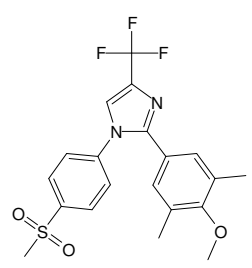

X084

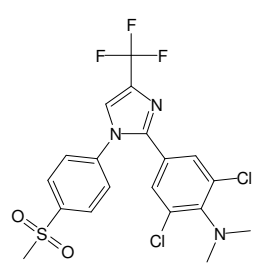

X085

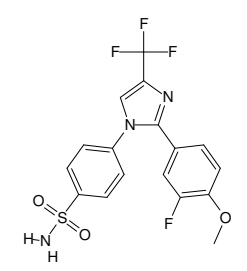

X086

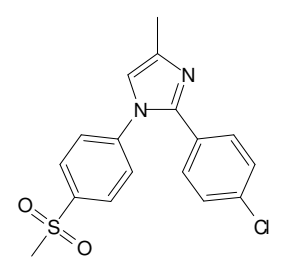




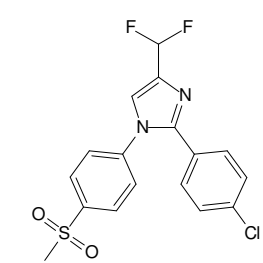

X088

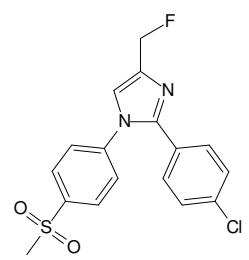

X089

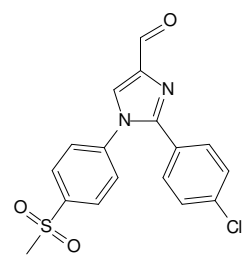

X090

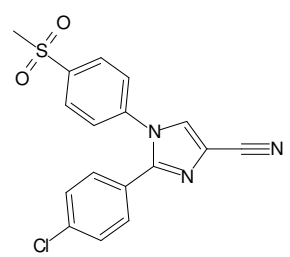

X091

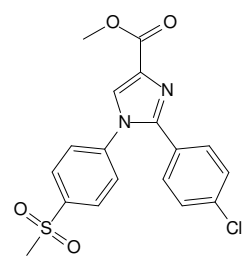

X092

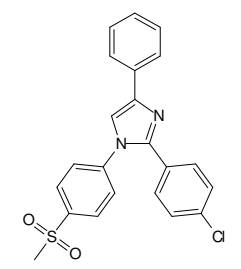

X093

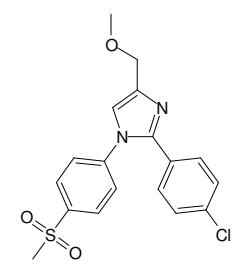

X094

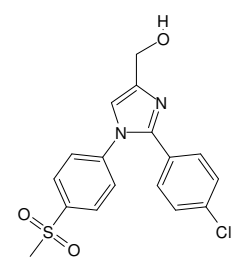

cox2 Clark

cox2 Clark

cox2 Clark

COX2 Clark

cox2 Clark

cox2 Clark

cox2 Clark COX2 Clark 


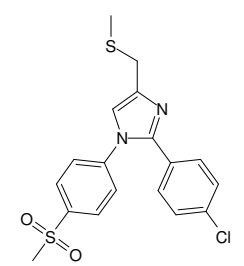

X096

X097
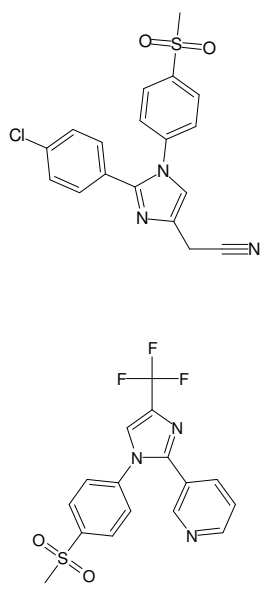

X098

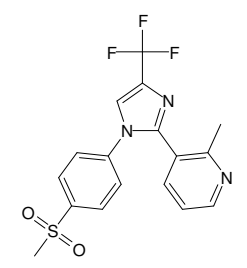

X099

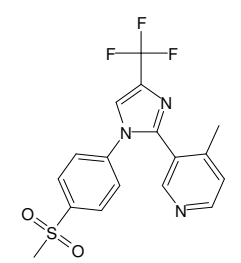

X100

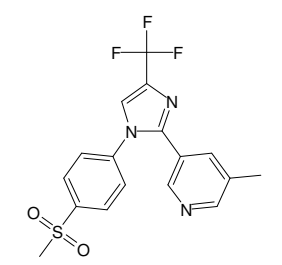

X101

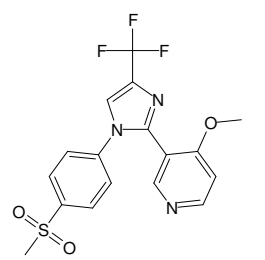

X102

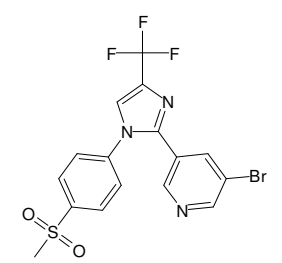

cox2 Clark

cox2 Clark

cox2 Clark

cox2 Clark

coX2 Clark

cox2 Clark

cox2 Clark COX2 Clark 


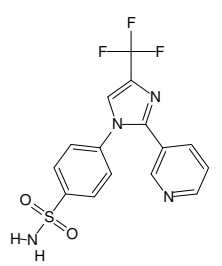

X104

cox2 Clark

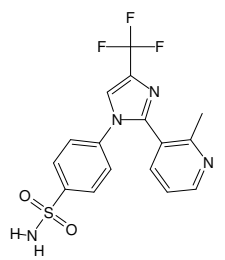

X105

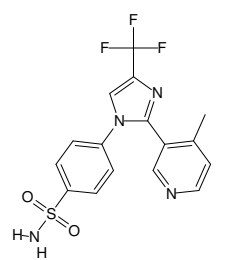

X106

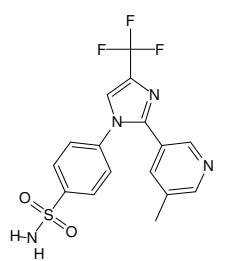

X107

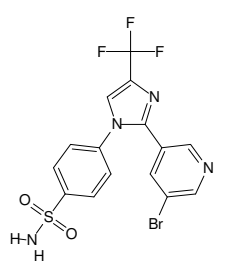

$\mathrm{X} 108$

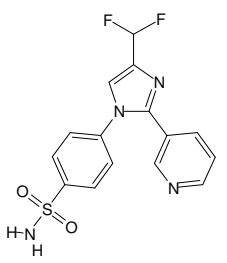

X109

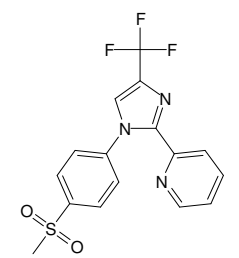

X110

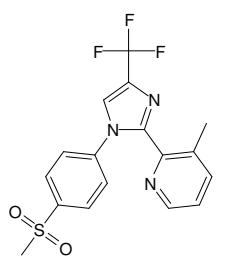




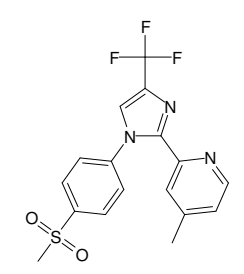

X112

cox2 Clark

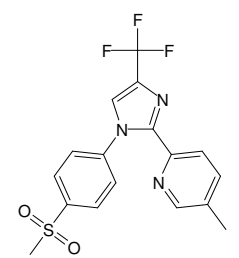

X113

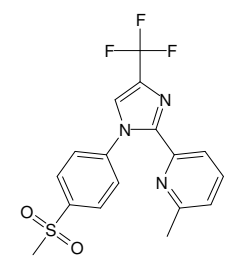

X114

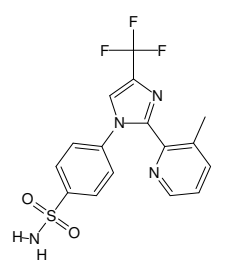

X115

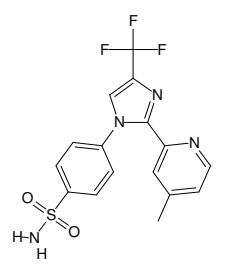

$\mathrm{X} 116$

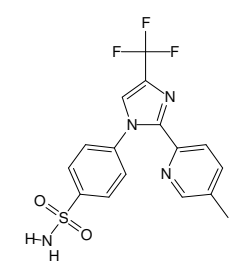

X117

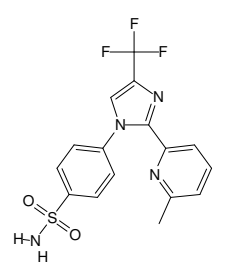

X118

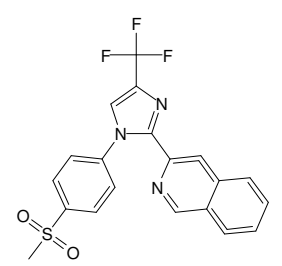




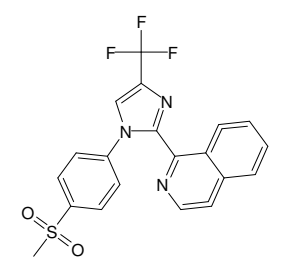

X120

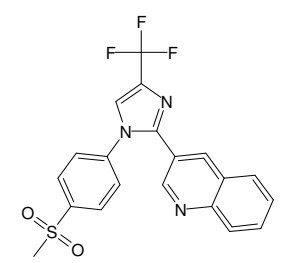

X121

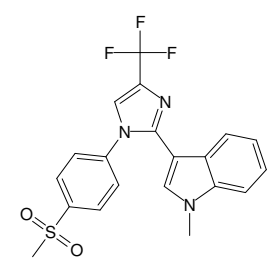

X122

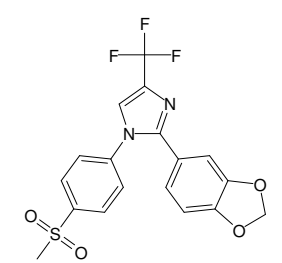

X123

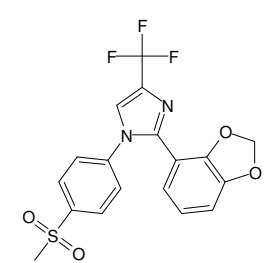

X124

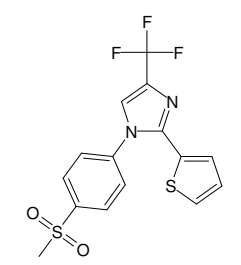

X125

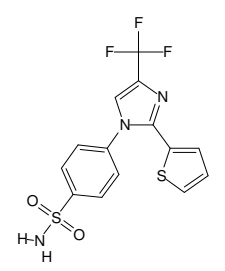

X126

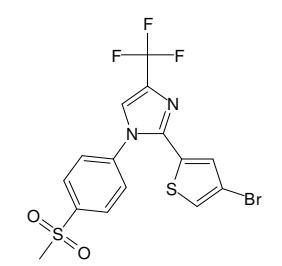




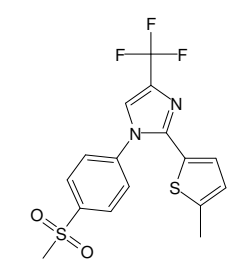

X128

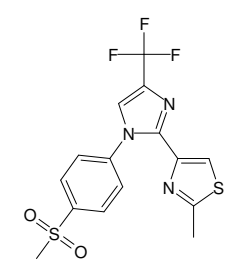

X129

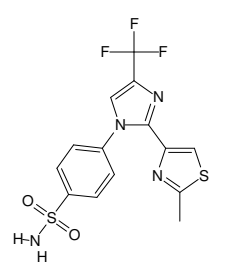

X130

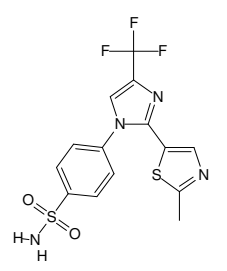

X131

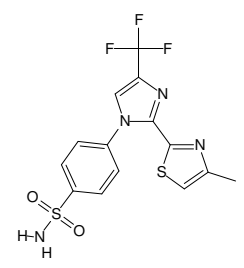

X132

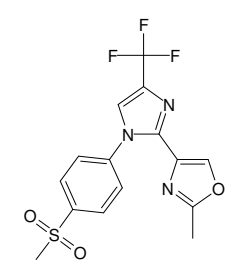

X133

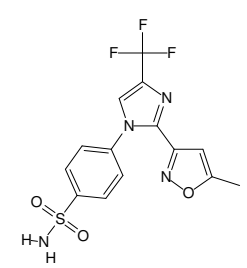

X134

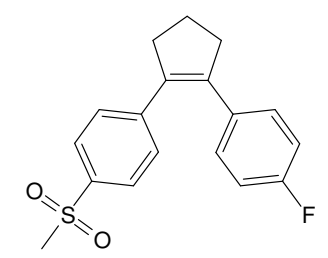




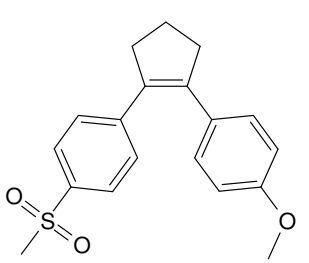

X136

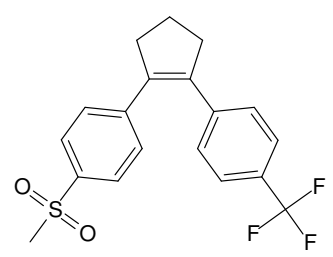

X137

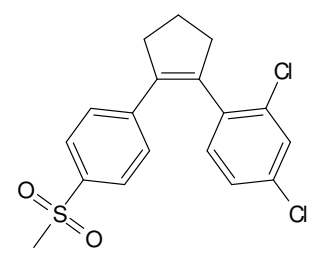

X138

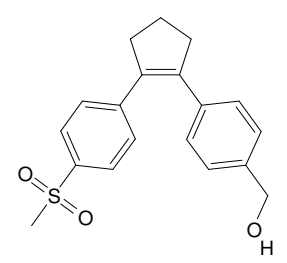

X139

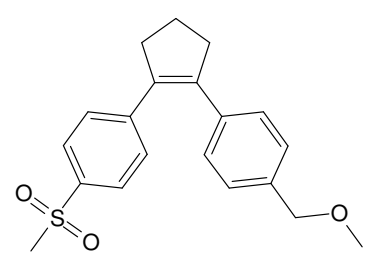

X140

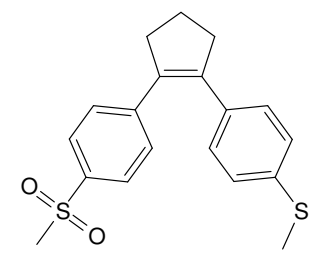

$\mathrm{X} 141$

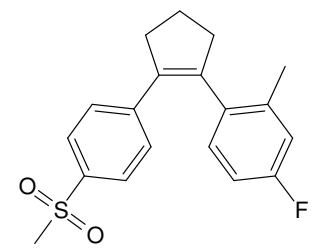

X142

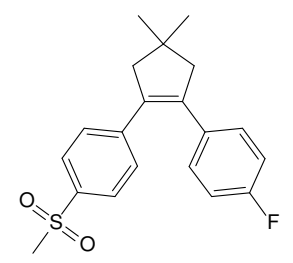

cox2 Clark

cox2 Clark

cox2 Clark

cox2 Clark

cox2 Clark

cox2 Clark

cox2 Clark cox2 Clark 


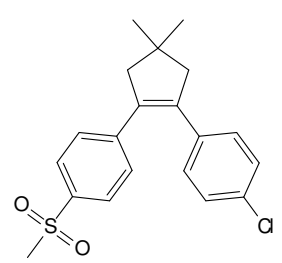

X144

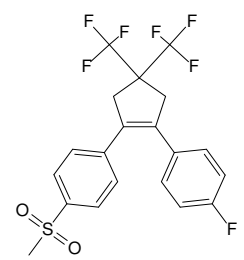

X145

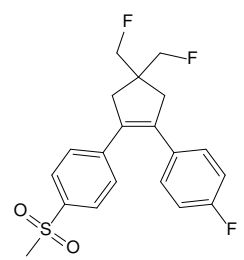

X146

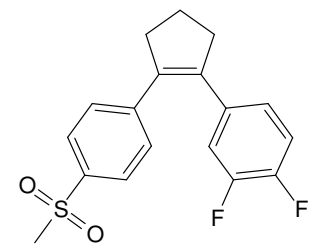

X147

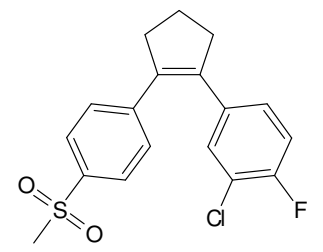

X148

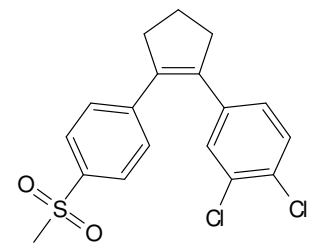

X149

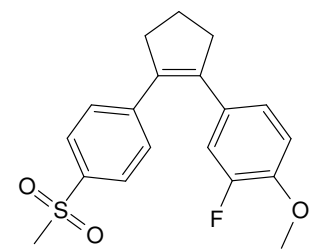

X150

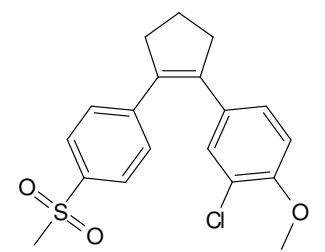




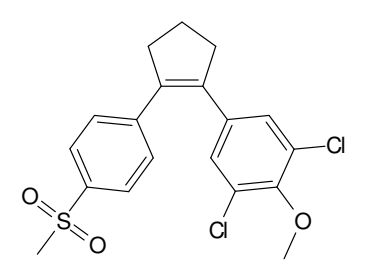

X152

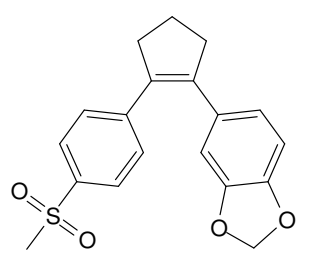

X153

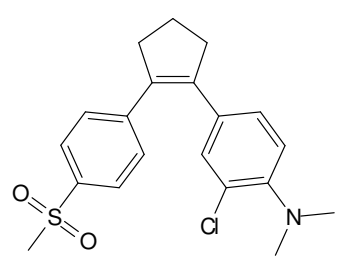

X154

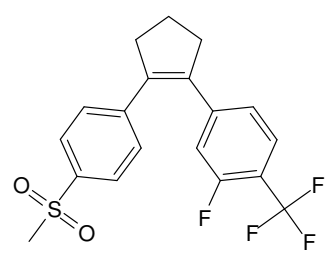

X155

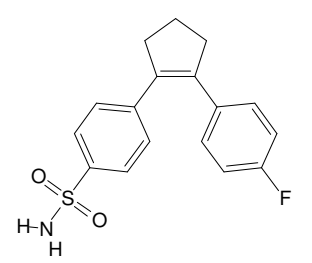

X156

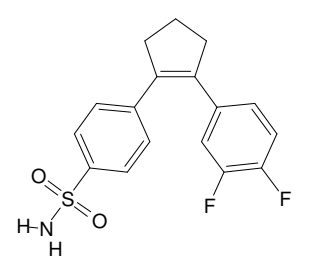

X157

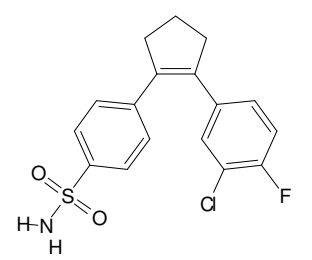

X158

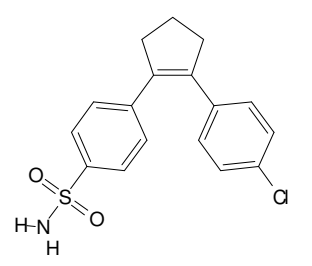

cox2 Clark

cox2 Clark

cox2 Clark

cox2 Clark

cox2 Clark

cox2 Clark

cox2 Clark

cox2 Clark 


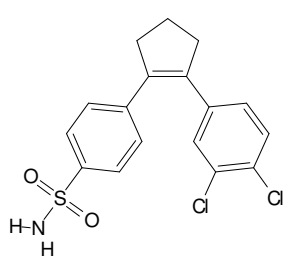

X160

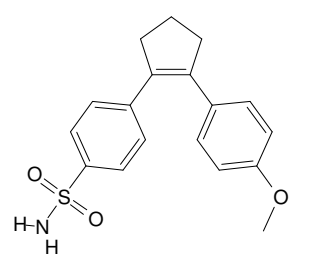

X161

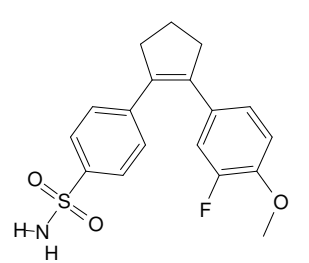

cox2 Clark

X162

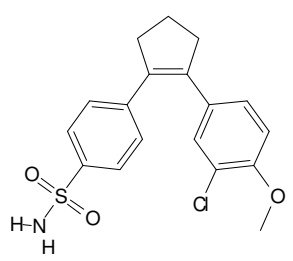

X163

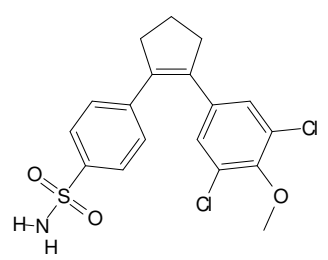

cox2 Clark

COX2 Clark

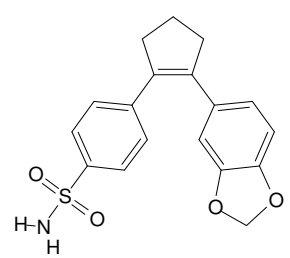

X165

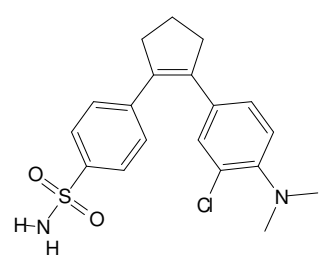

cox2 Clark

cox2 Clark

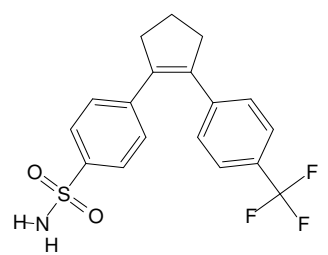




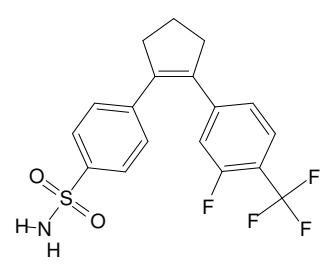

X168

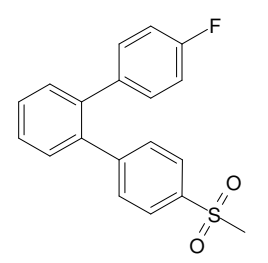

X169

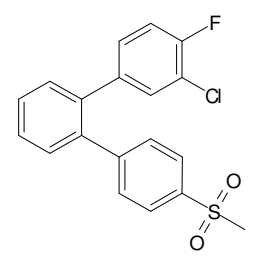

X170

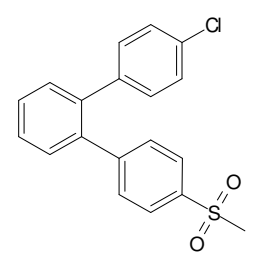

X171

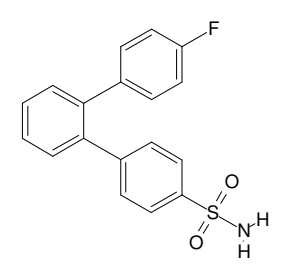

X172

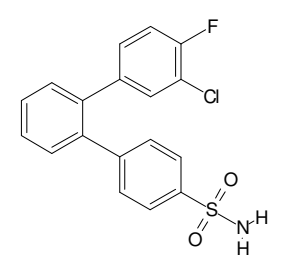

X173

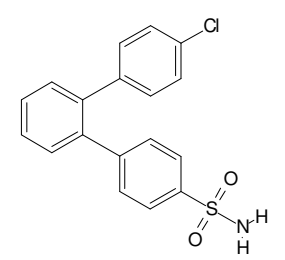

X174

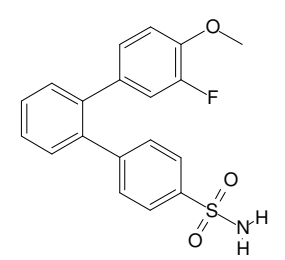




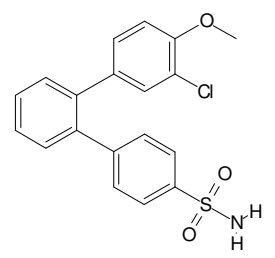

X176

cox2

Clark

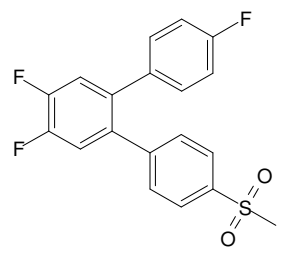

X177

cox2

Clark

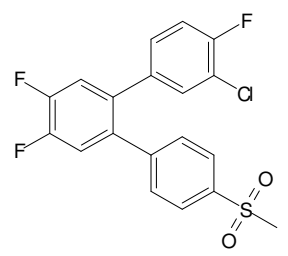

X178

COX2 Clark

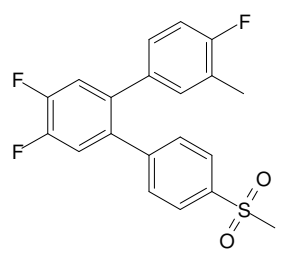

X179

cox2 Clark

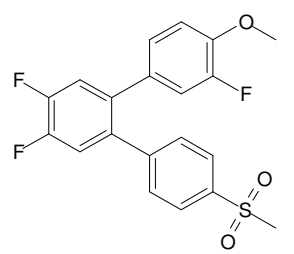

X180

cox2 Clark

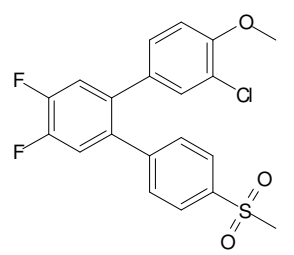

X181

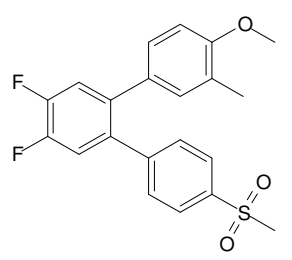

COX2 Clark

X182

cox2 Clark

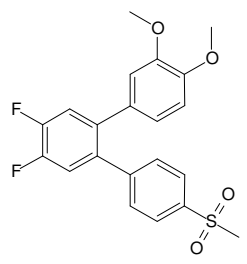

X183

COX2 Clark 


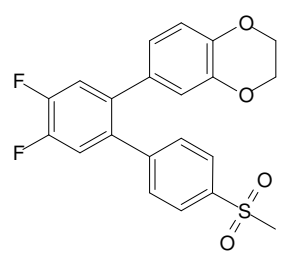

X184

COX2 Clark

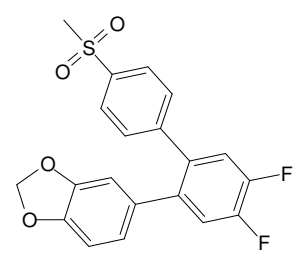

X185

COX2 Clark

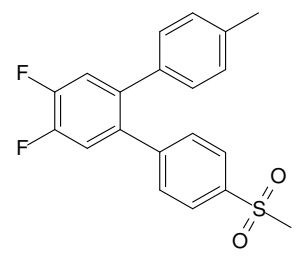

X186

COX2 Clark

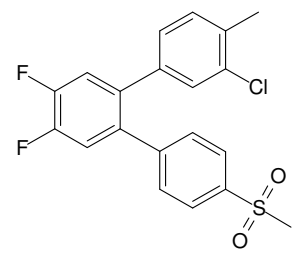

X187

COX2 Clark

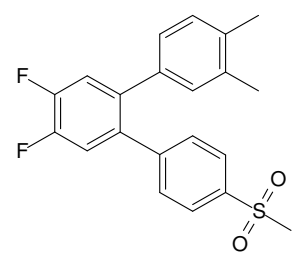

X188

COX2 Clark

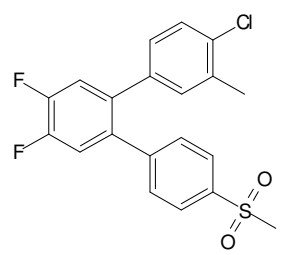

X189

COX2 Clark

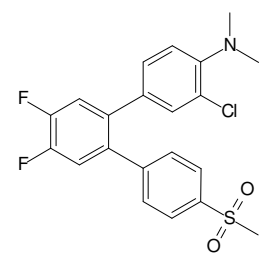

X190

CoX2 Clark

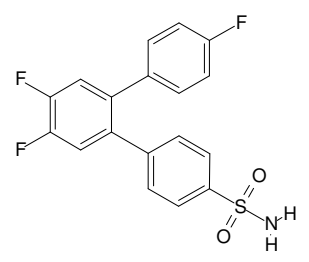

X191

COX2 Clark 


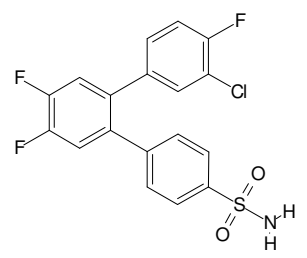

X192

coX2 Clark

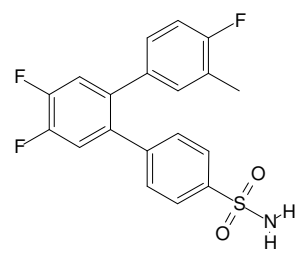

X193

coX2 Clark

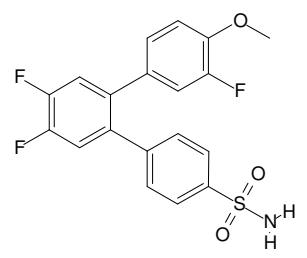

X194

COX2 Clark

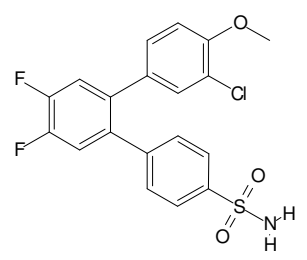

X195

COX2 Clark

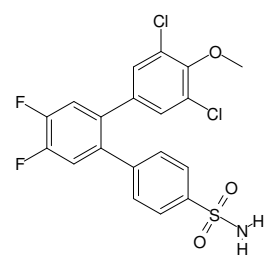

X196

COX2 Clark

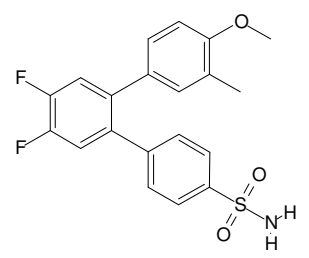

X197

COX2 Clark

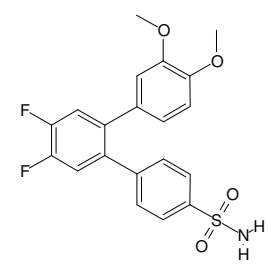

X198

CoX2 Clark

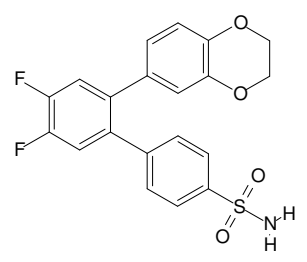

X199

COX2 Clark 


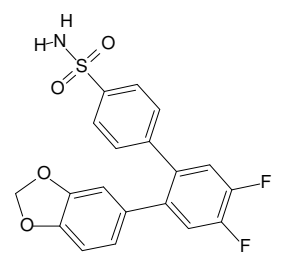

X200

cox2

Clark

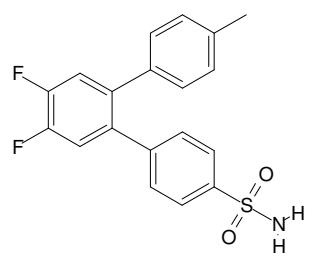

X201

coX2 Clark

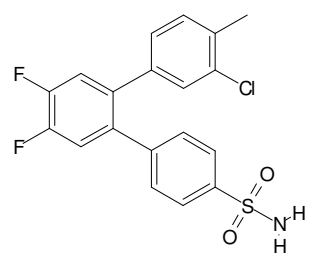

X202

coX2 Clark

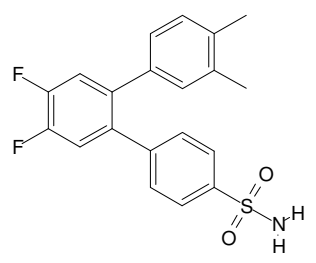

X203

cox2 Clark

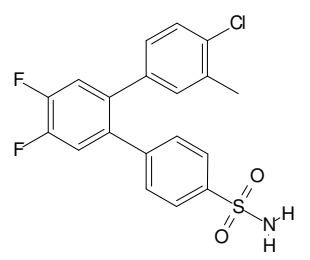

X204

cox2 Clark

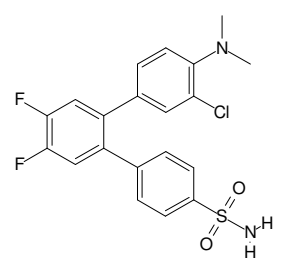

X205

coX2 Clark

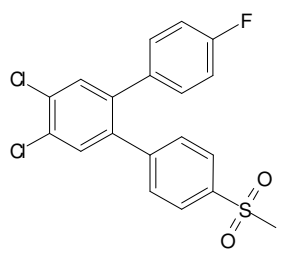

X206

coX2 Clark

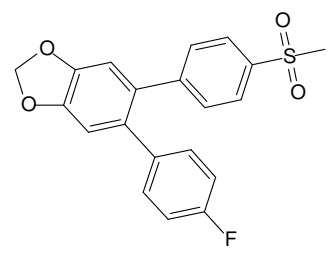

X207

coX2 Clark 


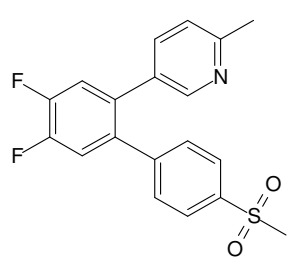

X208

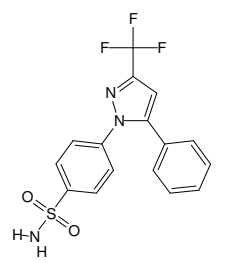

X209

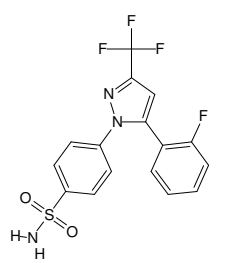

X210

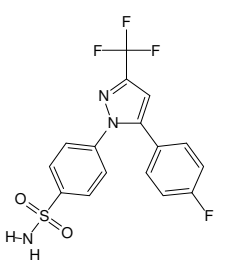

X211

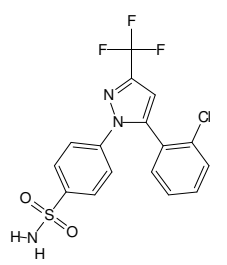

X212

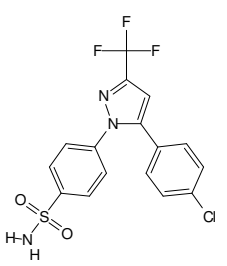

X213

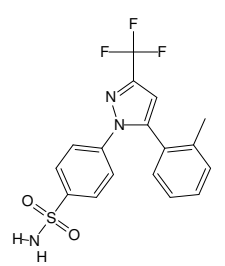

X214

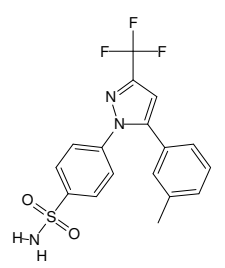




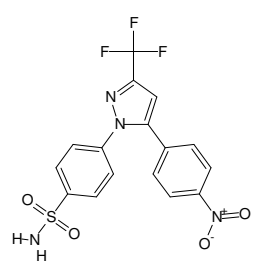

X216

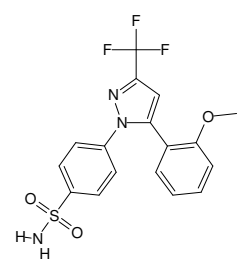

X217

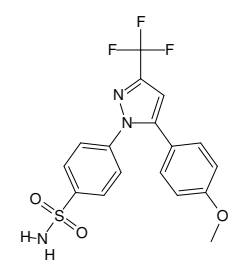

X218

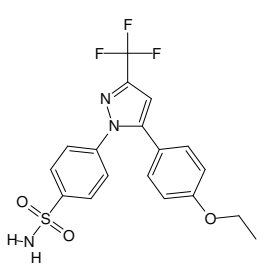

X219

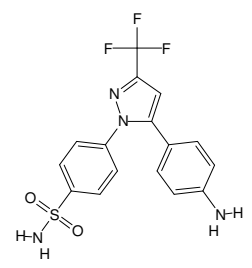

X220

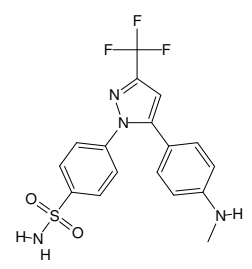

X221

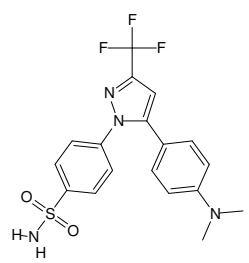

X222

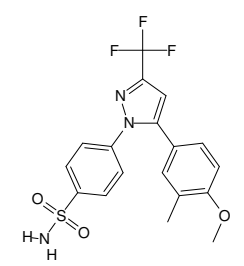




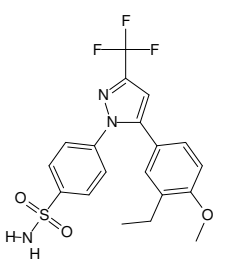

X224

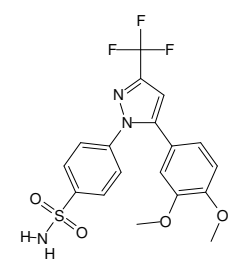

X225

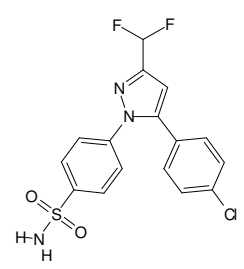

X226

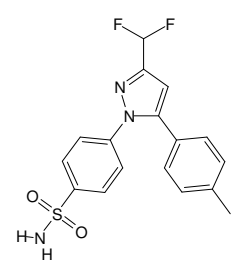

X227

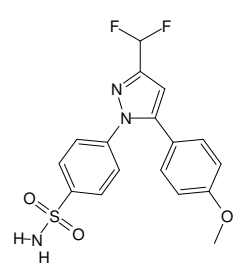

X228

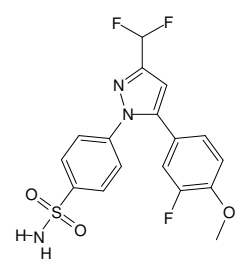

X229

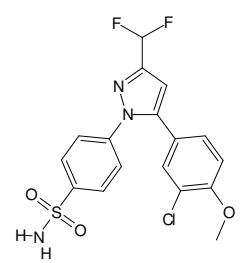

X230

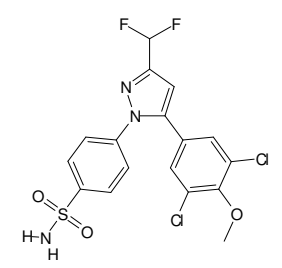




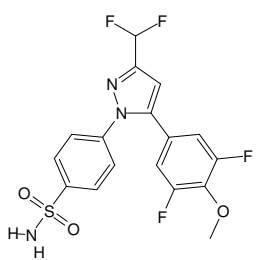

X232

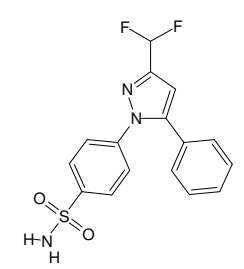

X233

X234
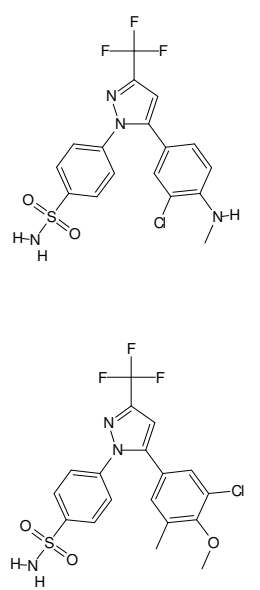

X235

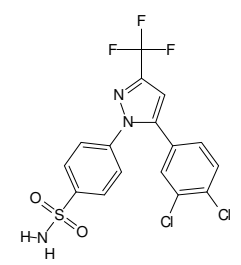

X236

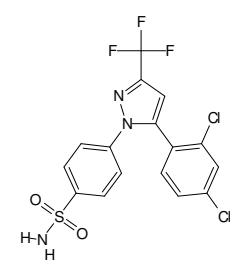

X237

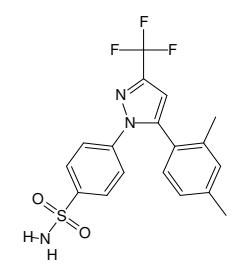

X238

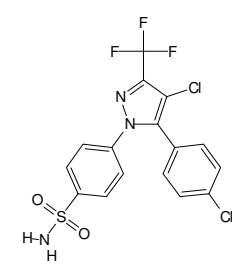

COX2 Clark

coX2 Clark

COX2 Clark

coX2 Clark

cox2 Clark

COX2 Clark

COX2 Clark COX2 Clark 


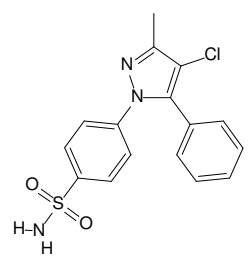

X240

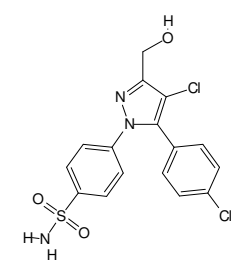

X241

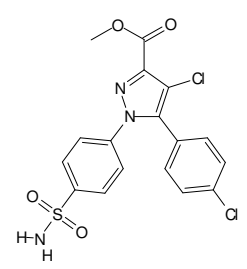

X242

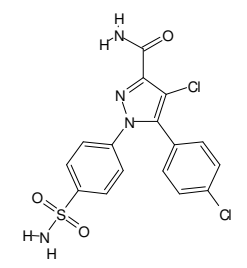

X243

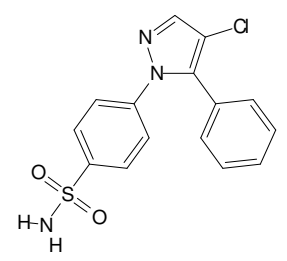

X244

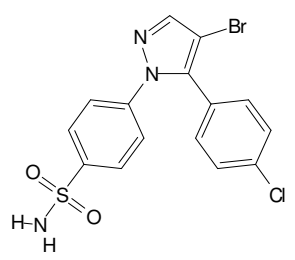

X245

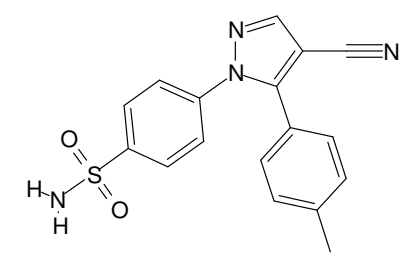

X246

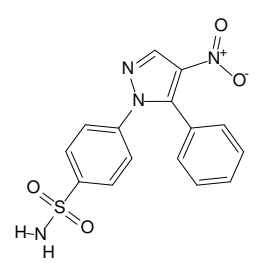




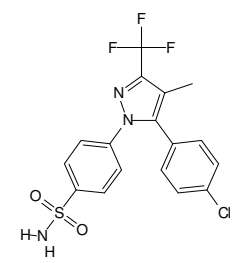

X248

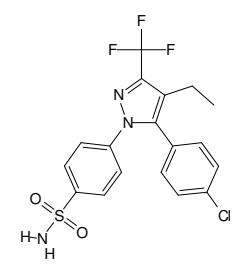

X249

$\mathrm{X} 250$
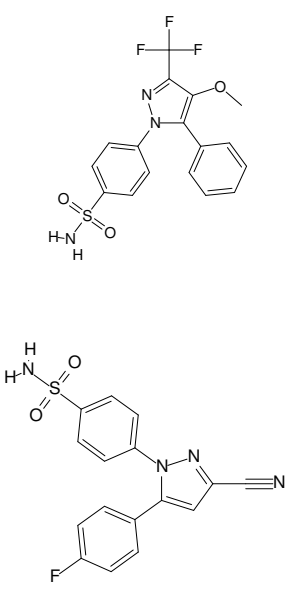

X251

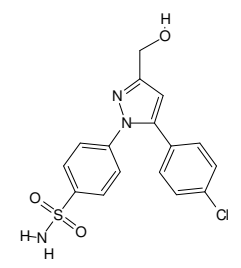

X252

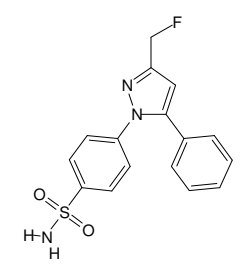

X253

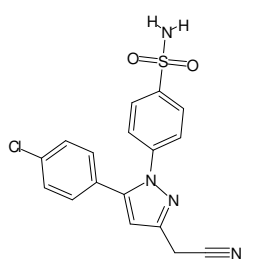

X254

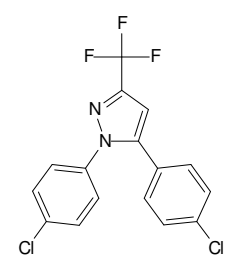

COX2 Clark

cox2 Clark

cox2 Clark

coX2 Clark

coX2 Clark

coX2 Clark

cox2 Clark COX2 Clark 


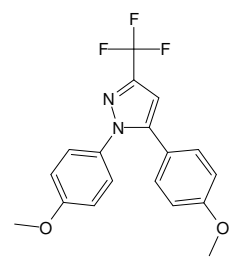

X256_A

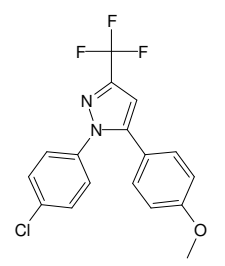

X257_A

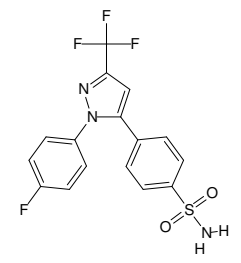

X258_A

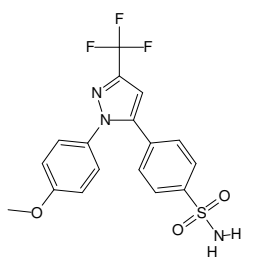

X259 A

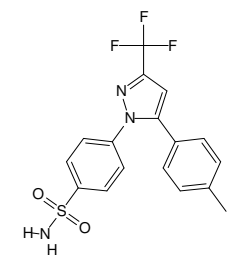

X260

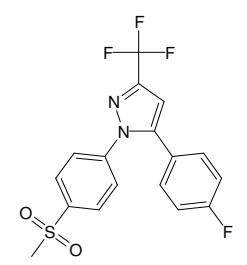

X261

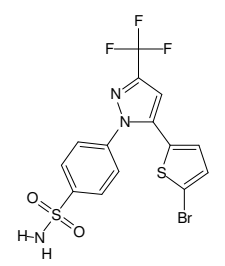

X262

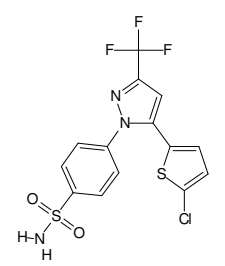

cox2 Clark

cox2 Clark

cox2 Clark

COX2 Clark

COX2 Clark

cox2 Clark

cox2 Clark COX2 Clark 


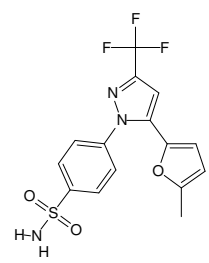

X264

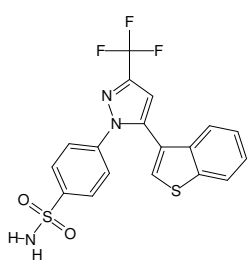

X265

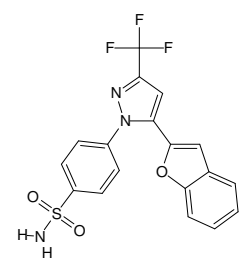

X266

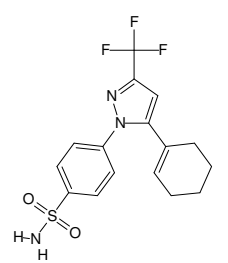

X267

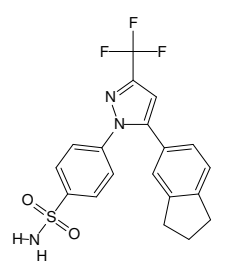

X268

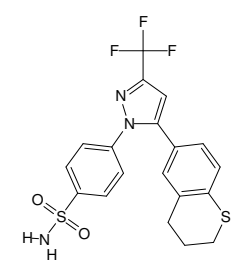

X269

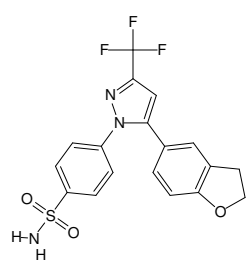

X270

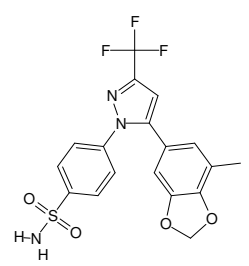

X271_A

COX2 Clark 


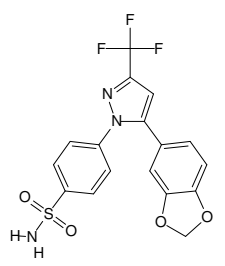

X272

cox2 Clark

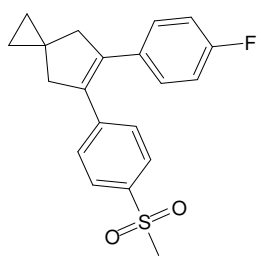

X273

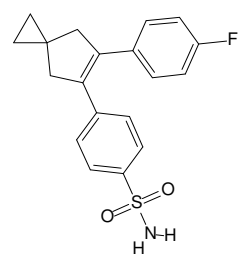

cox2 Clark

X274

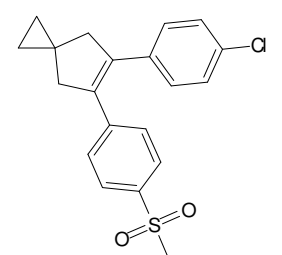

X275

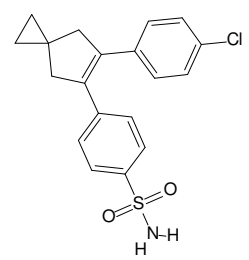

COX2 Clark

cox2 Clark

COX2 Clark

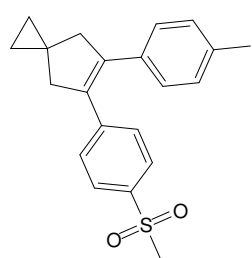

X277

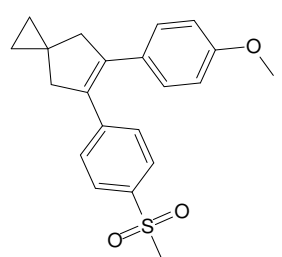

X278

COX2 Clark

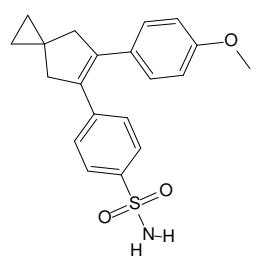




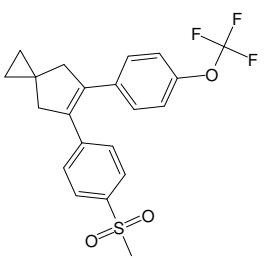

X280

cox2 Clark

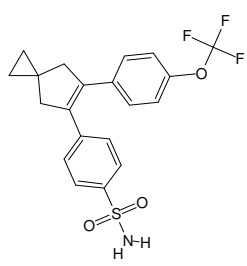

X281

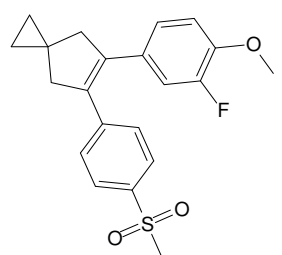

X282

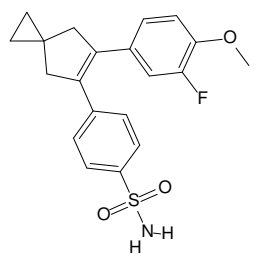

X283

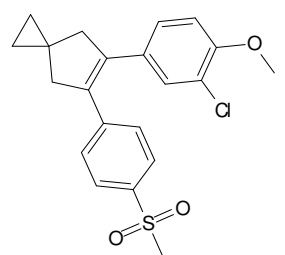

X284

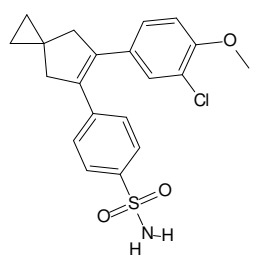

X285

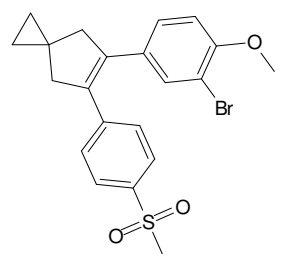

X286

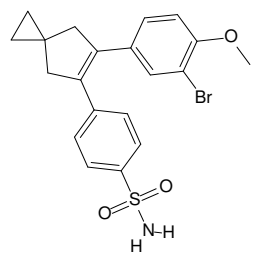

COX2 Clark 


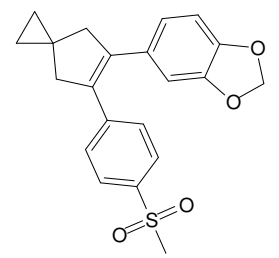

X288

cox2 Clark

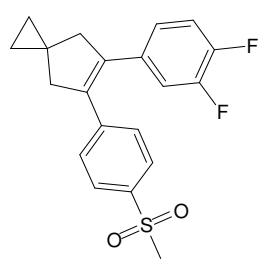

X289

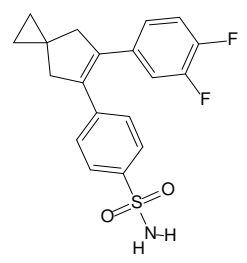

X290

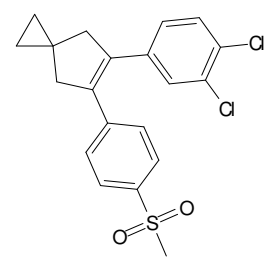

X291

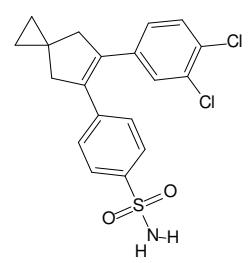

X292

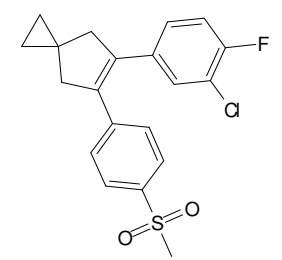

X293

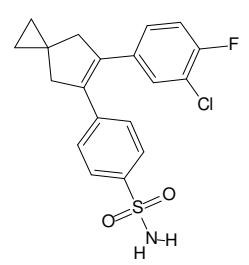

X294

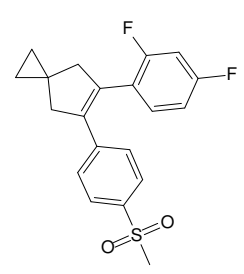




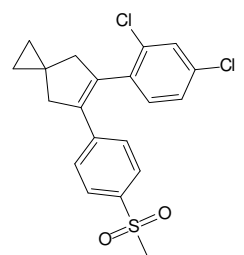

X296

cox2 Clark

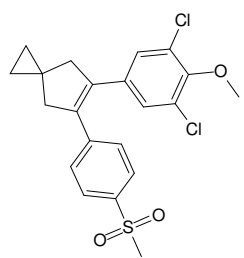

X297

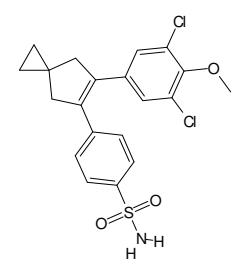

cox2 Clark

X298

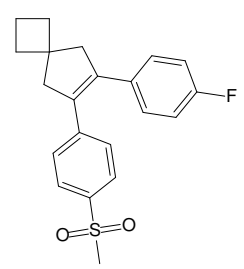

cox2 Clark

X299

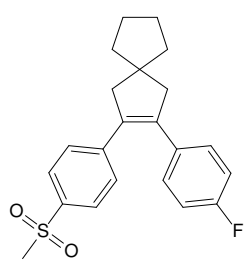

X300

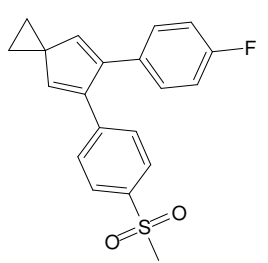

X301

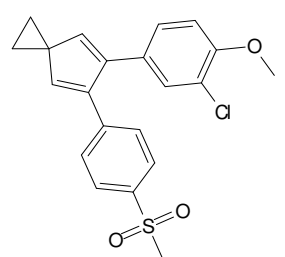

X302

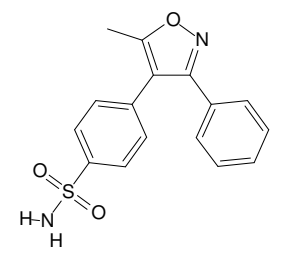




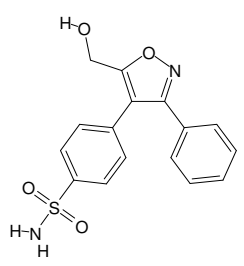

X304

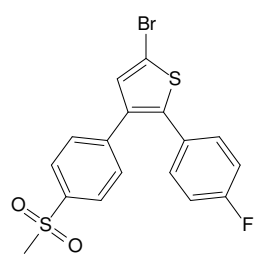

X305

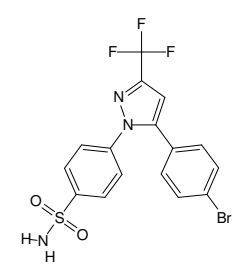

1CX2_lig

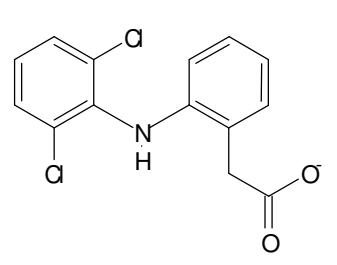

1PXX_lig

coX2 Clark

cox2 Clark

COX2 PDB

COX2 PDB<smiles>CC(C(=O)O)c1ccc(-c2ccccc2)c(F)c1</smiles>

3PGH_lig

COX2 PDB

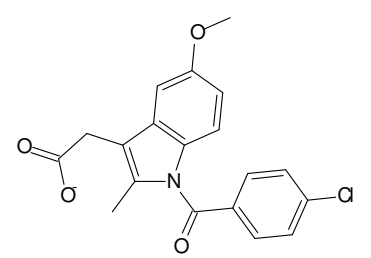

4COX_lig

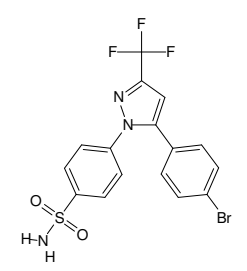

6COX_lig<smiles></smiles>

A_1_1

DHFR Otzen 
<smiles>Nc1nc(N)c(Cc2ccc(Br)c3ccccc23)c[nH+]1</smiles>

A_1_10

DHFR Otzen<smiles></smiles>

A_1_12<smiles>Nc1nc(N)c(OCc2ccccc2Cl)c[nH+]1</smiles>

A_1_2<smiles></smiles>

A_1_4<smiles>Nc1nc(N)c(OCc2c(Cl)cccc2Cl)c[nH+]1</smiles>

A_1_5<smiles></smiles>

A_1_6<smiles></smiles>

A_1_8<smiles></smiles>

A_1_9

DHFR Otzen 
<smiles>Cc1nc(N)nc(N)c1Cc1ccccc1</smiles>

A_2_1

DHFR Otzen<smiles></smiles>

A_2_4<smiles></smiles>

C_1_1

DHFR Otzen<smiles></smiles>

C_1_11

DHFR Otzen<smiles></smiles>

C_1_2<smiles></smiles>

DHFR Otzen

DHFR Otzen<smiles></smiles>

C_1_4

DHFR Otzen<smiles></smiles> 
<smiles></smiles>

C_1_7<smiles></smiles>

C_1_9<smiles></smiles>

D_1_1<smiles></smiles>

D_1_11<smiles></smiles>

D_1_9<smiles></smiles>

E_1_1<smiles></smiles>

F_1_1<smiles></smiles> 


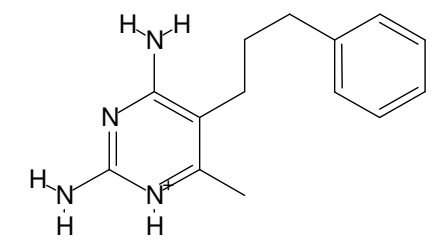

G_2_1<smiles>Nc1nc(N)c(CNc2ccccc2)c[nH+]1</smiles>

H_1_1<smiles></smiles>

J_1_1

DHFR Otzen<smiles></smiles>

Rol2-6099<smiles></smiles>

TMP

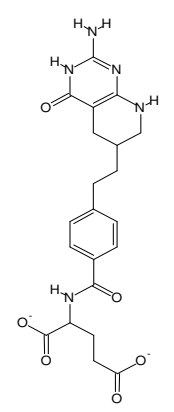

5_10_deazafolat

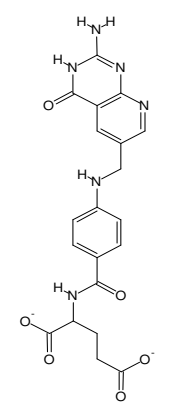

DHFR PDB 


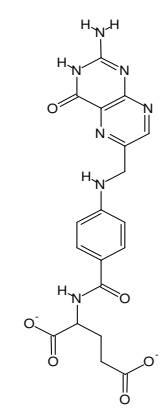

folat

DHFR PDB<smiles>CN(Cc1cnc2nc(N)nc(N)c2n1)c1ccc(C(=O)NC(CCC(=O)[O-])C(=O)[O-])cc1</smiles>

MTX

DHFR PDB<smiles>CC1CCc2c(N)nc(N)[nH+]c2C1</smiles>

MAC_0002654<smiles>CC1Cc2[nH+]c(N)nc(N)c2CC1C</smiles>

MAC_0002655

DHFR Zolli-Juran

DHFR Zolli-Juran

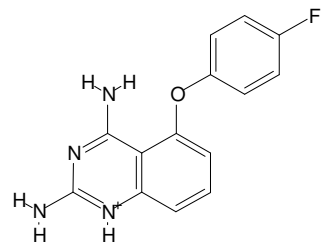

MAC 0030856

DHFR Zolli-Juran

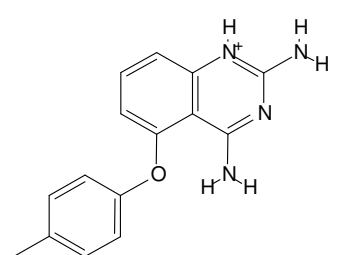

MAC 0030857 


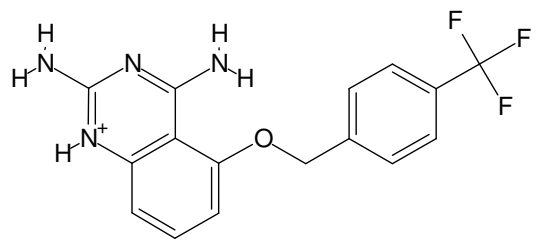

MAC_0030861

DHFR Zolli-Juran

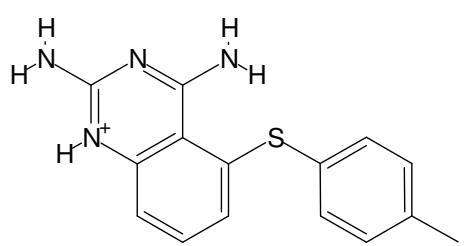

MAC_0030862

DHFR Zolli-Juran

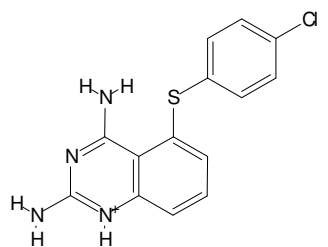

MAC_0030863

DHFR Zolli-Juran

DHFR Zolli-Juran

MAC 0033238
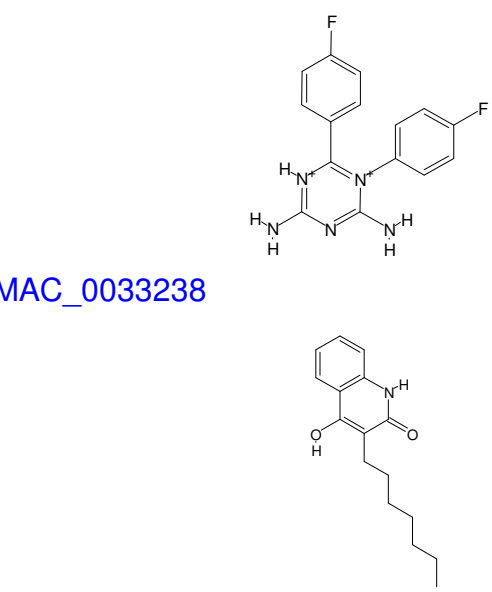

MAC 0036664

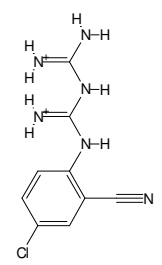

MAC_0038968

DHFR Zolli-Juran

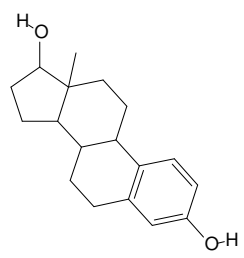

1ERE_lig

ERa PDB

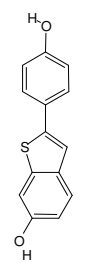




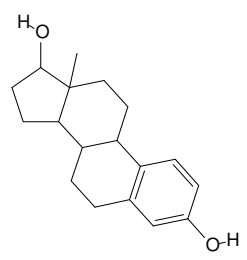

1GWR_lig

ERa PDB

1L2I_lig

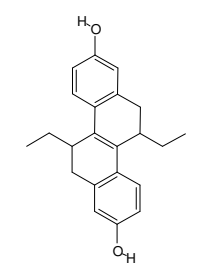

ERa PDB

3ERD_lig<smiles>CCC(=C(CC)c1ccc(O)cc1)c1ccc(O)cc1</smiles><smiles>CC/C(=C(/CC)c1ccc(O)cc1)c1ccc(O)cc1</smiles>

E2_1

ERa Shi

ABS<smiles>C[C@H]1CCCC(=O)CCC/C=C/c2cc(O)cc(O)c2C(=O)O1</smiles>

E2_10

ERa Shi

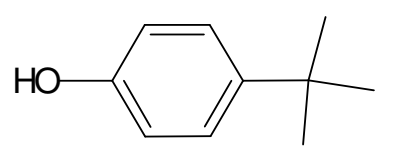

E2_100

ERa Shi<smiles>Clc1ccc(-c2ccccc2Cl)cc1</smiles>

E2_101

ERa Shi<smiles>Cc1ccc(O)c(Cl)c1</smiles> 


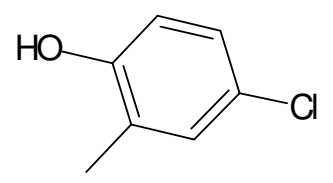

E2_103

ERa Shi

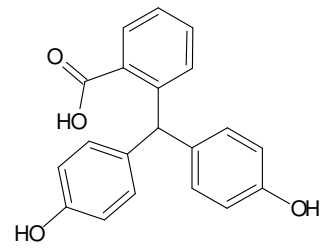

E2_104

ERa Shi<smiles>O=C1CC(c2ccccc2)Oc2cc(O)ccc21</smiles>

E2_105

ERa Shi<smiles>CCc1cccc(O)c1</smiles>

E2_106

ERa Shi

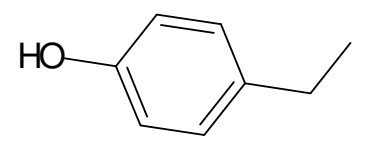

E2_108

ERa Shi

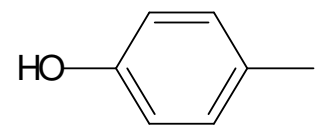

E2_109

ERa Shi<smiles>O=c1oc2cc(O)ccc2c2oc3cc(O)ccc3c12</smiles>

E2_11

ERa Shi

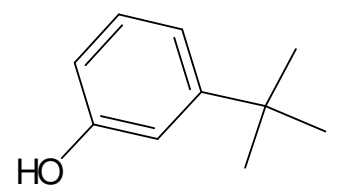

E2_110

ERa Shi<smiles>Oc1ccc(-c2c(Cl)cc(Cl)cc2Cl)cc1</smiles>

E2_12

ERa Shi 
RAC

(N)

E2 13<smiles>O=c1c(O)c(-c2ccc(O)cc2)oc2ccc(O)cc12</smiles>

E2_14<smiles>O=c1c(-c2ccc(O)cc2)coc2cc(O)cc(O)c12</smiles>

E2_15

ABS

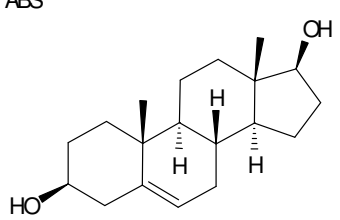

E2 16

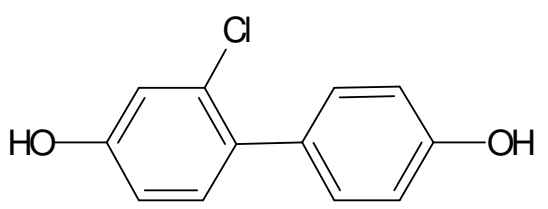

E2_17<smiles>Oc1ccc(-c2cc(Cl)c(Cl)c(Cl)c2Cl)cc1</smiles>

E2 18

ABS

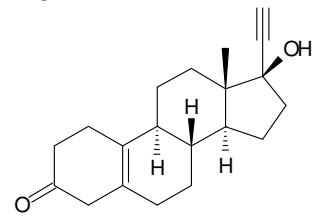

E2_19

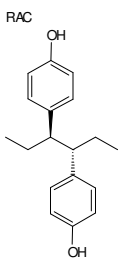

E2 2
ERa Shi

ERa Shi

ERa Shi

ERa Shi

ERa Shi

ERa Shi

ERa Shi

ERa Shi 


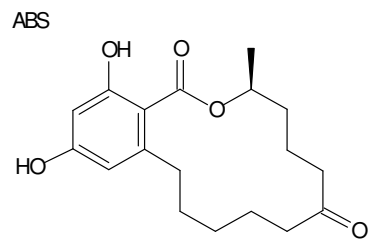

E2_20

ERa Shi<smiles>Oc1ccc(C2COc3cc(O)ccc3C2)cc1</smiles>

E2_21

ERa Shi<smiles>O=c1cc(-c2ccc(O)cc2)oc2ccc(O)cc12</smiles>

E2_22<smiles>Oc1ccc(-c2c(Cl)cc(Cl)cc2Cl)c(Cl)c1</smiles>

ERa Shi

E2_23

ERa Shi<smiles>Oc1ccc(-c2c(Cl)cc(Cl)c(Cl)c2Cl)c(Cl)c1</smiles>

E2_24

ERa Shi<smiles>Oc1ccc(-c2c(Cl)cccc2Cl)cc1</smiles>

E2_25

ERa Shi<smiles>O=C(CCc1ccc(O)cc1)c1c(O)cc(O)cc1O</smiles>

E2_26

ERa Shi<smiles>Oc1ccc(-c2c(Cl)cc(Cl)cc2Cl)cc1Cl</smiles> 
<smiles>Oc1ccc(-c2c(Cl)cc(Cl)c(Cl)c2Cl)cc1Cl</smiles>

E2_28

ERa Shi<smiles>Oc1ccc(-c2cc(Cl)ccc2Cl)cc1</smiles>

E2_29

ERa Shi

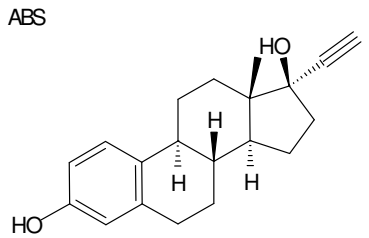

E2_3<smiles>Oc1ccc(-c2cc(Cl)c(Cl)c(Cl)c2Cl)cc1Cl</smiles>

E2_30

ERa Shi<smiles>Oc1c(Cl)cc(-c2cc(Cl)c(Cl)c(Cl)c2Cl)c(Cl)c1Cl</smiles>

E2_31<smiles>Oc1ccc(-c2cc(Cl)c(Cl)c(Cl)c2Cl)c(Cl)c1</smiles>

E2_32

ERa Shi<smiles>Oc1cc(Cl)c(-c2c(Cl)c(Cl)c(Cl)c(Cl)c2Cl)cc1Cl</smiles>

E2_33

ERa Shi<smiles>CCCCCCCCCc1ccc(O)cc1</smiles> 


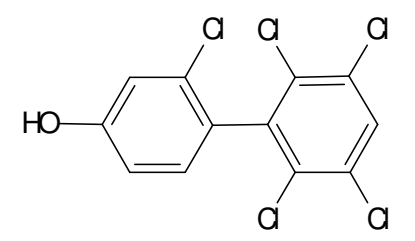

E2_35

ERa Shi<smiles>Oc1c(Cl)cc(-c2cc(Cl)c(Cl)c(Cl)c2Cl)c(Cl)c1O</smiles>

E2_36

ERa Shi<smiles>O=c1cc(-c2ccc(O)cc2)oc2cc(O)cc(O)c12</smiles>

E2_37

ERa Shi<smiles>O=c1c(O)c(-c2ccc(O)cc2)oc2cc(O)cc(O)c12</smiles>

E2_38

ERa Shi<smiles>Oc1c(Cl)cc(-c2ccc(Cl)c(Cl)c2Cl)c(Cl)c1Cl</smiles>

E2_39

ERa Shi<smiles>C/C=C(C(=C/C)\c1ccc(O)cc1)/c1ccc(O)cc1</smiles>

E2_4

ERa Shi<smiles>O=c1c(-c2ccc(O)cc2)coc2cc(O)ccc12</smiles>

E2_40

ERa Shi<smiles>Oc1ccc(-c2c(Cl)c(Cl)cc(Cl)c2Cl)cc1Cl</smiles>

E2 41

ERa Shi 

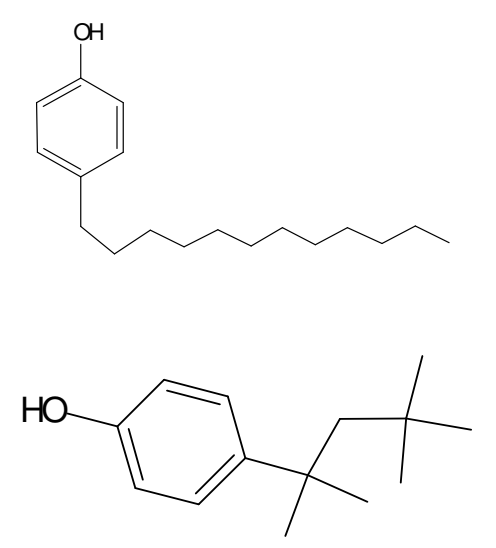

E2_43<smiles>Oc1cc(O)c(-c2c(Cl)cc(Cl)cc2Cl)c(Cl)c1</smiles>

E2_44

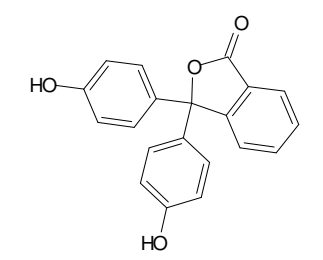

E2_45

$$
\text { (a) }
$$

E2_46<smiles>Oc1cc(Cl)c(-c2c(Cl)cccc2Cl)c(Cl)c1Cl</smiles>

E2_47<smiles>Oc1c(Cl)cc(-c2cc(Cl)c(Cl)cc2Cl)c(Cl)c1Cl</smiles>

E2_48<smiles>Oc1c(Cl)cc(-c2ccc(Cl)c(Cl)c2)c(Cl)c1Cl</smiles> 


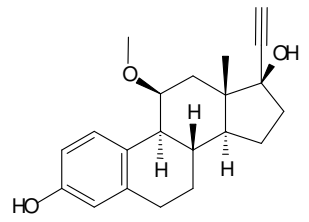

E2_5<smiles>CC(C)(c1ccc(O)cc1)c1ccc(O)cc1</smiles>

E2_50<smiles>O=C1CC(c2ccc(O)cc2)Oc2cc(O)cc(O)c21</smiles>

E2 51<smiles>Oc1ccc(-c2c(Cl)c(Cl)c(O)c(Cl)c2Cl)cc1</smiles>

E2_52

ERa Shi<smiles>Oc1ccc(-c2ccc(Cl)cc2)cc1</smiles>

E2_53

ERa Shi

ABS

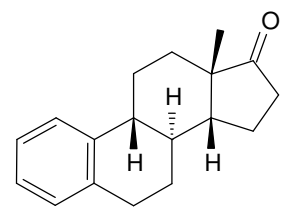

E2_54<smiles>O=c1c(O)c(-c2ccc(O)c(O)c2)oc2cc(O)ccc12</smiles>

E2_55

ERa Shi<smiles>O=c1c(-c2ccc(O)c(O)c2)coc2cc(O)ccc12</smiles> 
<smiles>COc1ccc(-c2coc3cc(O)cc(O)c3c2=O)cc1</smiles>

E2_57<smiles>O=C(/C=C/c1ccccc1)c1ccc(O)cc1</smiles>

E2_58

ERa Shi<smiles>Oc1ccc(Cl)cc1Cc1cc(Cl)ccc1O</smiles>

E2_59

ERa Shi

ABS

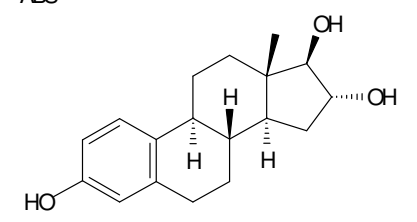

E2_6

ERa Shi<smiles>Oc1ccc(-c2ccc(O)cc2)cc1</smiles>

E2_60

ERa Shi<smiles>Oc1c(Cl)cc(-c2ccc(Cl)c(Cl)c2Cl)cc1Cl</smiles>

E2_61

ERa Shi<smiles>Oc1ccc(Cc2ccc(O)cc2)cc1</smiles>

E2_62

ERa Shi<smiles>O=C(OCc1ccccc1)c1ccc(O)cc1</smiles>

E2_63

ERa Shi 
<smiles>O=C(/C=C/c1ccc(O)cc1)c1ccccc1</smiles>

E2_64

ERa Shi<smiles>Oc1cc(Cl)c(-c2c(Cl)cc(Cl)cc2Cl)c(Cl)c1Cl</smiles>

E2_65<smiles>O=C(c1ccccc1)c1ccc(O)cc1O</smiles>

E2_66

ERa Shi

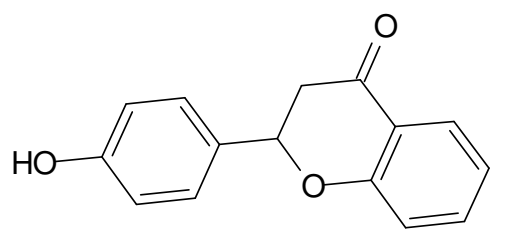

E2_67

ERa Shi

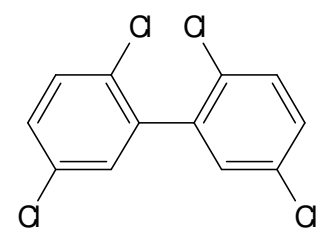

E2_68

ERa Shi

E2_69

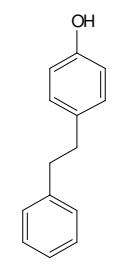

ERa Shi 
<smiles>Oc1cc(Cl)c(-c2c(Cl)ccc(Cl)c2Cl)c(Cl)c1Cl</smiles>

E2_71

ERa Shi<smiles>O=c1c(O)c(-c2cc(O)c(O)c(O)c2)oc2cc(O)cc(O)c12</smiles>

E2_72

ERa Shi

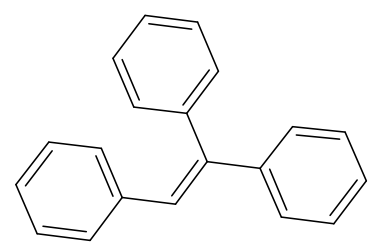

E2_73

ERa Shi<smiles>O=C1CC(c2cccc(O)c2)Oc2ccccc21</smiles>

E2_74

ERa Shi<smiles>O=C(/C=C/c1ccccc1)c1ccccc1</smiles>

E2_75

ERa Shi<smiles>Oc1cc(O)c(-c2cc(Cl)c(Cl)cc2O)cc1O</smiles>

E2_76

ERa Shi<smiles>Clc1ccc(C(c2ccccc2Cl)C(Cl)(Cl)Cl)cc1</smiles>

E2_77

ERa Shi<smiles>CCCCCCCOc1ccc(O)cc1</smiles>

E2_78

ERa Shi 
<smiles>COc1ccc(-c2coc3cc(O)ccc3c2=O)cc1</smiles>

E2_79

ERa Shi

ABS

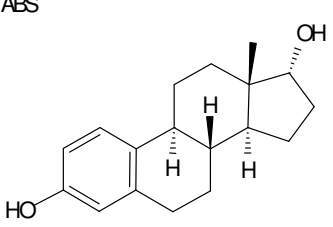

E2_8

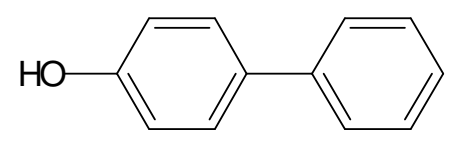

ERa Shi

E2_80

ERa Shi

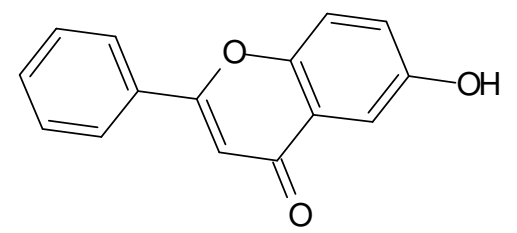

E2_81

ERa Shi<smiles>O=S(=O)(c1ccc(O)cc1)c1ccc(O)cc1</smiles>

E2_82

ERa Shi<smiles>CCCCOC(=O)c1ccc(O)cc1</smiles>

E2_83

ERa Shi<smiles>CC(CCC(=O)O)(c1ccc(O)cc1)c1ccc(O)cc1</smiles>

E2_84

ERa Shi<smiles>CCOC(=O)c1ccc(O)cc1</smiles> 


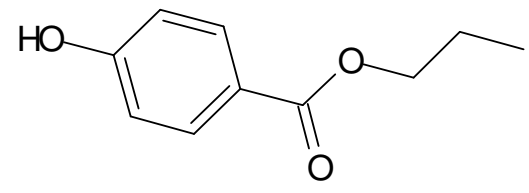

E2_86

ERa Shi

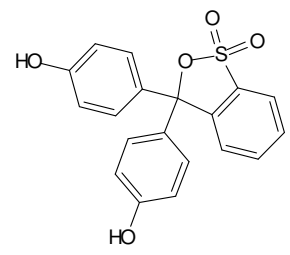

E2_87

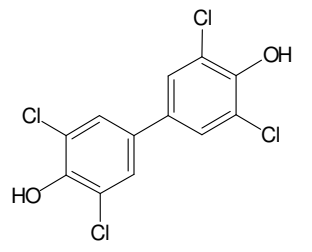

E2_88

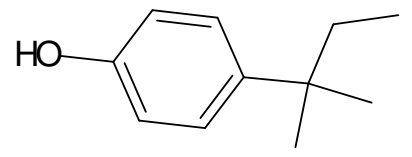

E2_89

ERa Shi

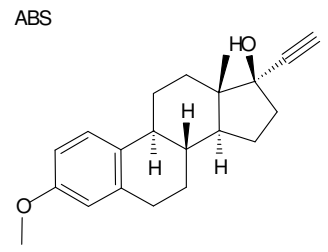

E2_9

ERa Shi

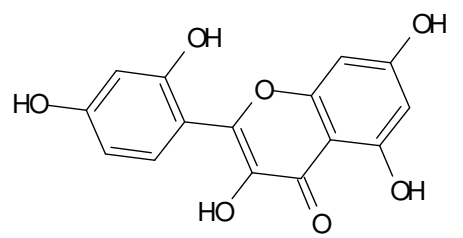

E2_90

ERa Shi<smiles>O=c1cc(-c2ccccc2)oc2cc(O)c(O)c(O)c12</smiles>

E2_91

ERa Shi

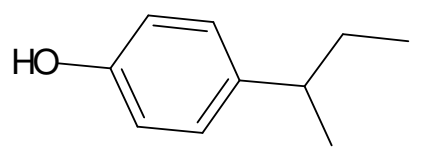

E2_92

ERa Shi 
<smiles>Cc1cc(O)ccc1Cl</smiles>

E2_93

ERa Shi

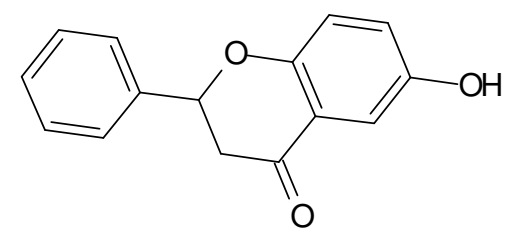

E2_94

ERa Shi

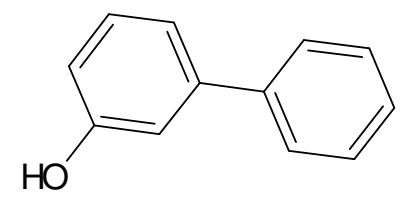

E2_95

ERa Shi

E2_96

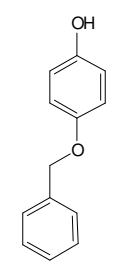

ERa Shi

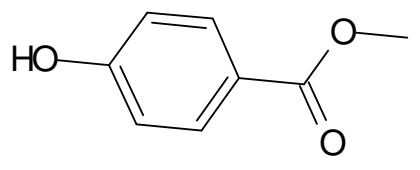

E2_97

ERa Shi

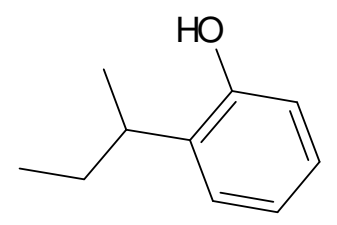

E2_98

ERa Shi

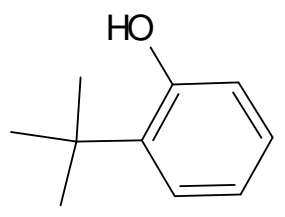

E2_99

ERa Shi<smiles>Oc1ccc(-c2ccc(O)cc2)cc1</smiles>

E1_1

ERa Sippl 


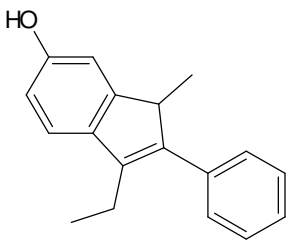

E1_10

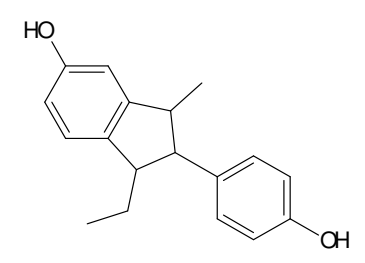

E1_11

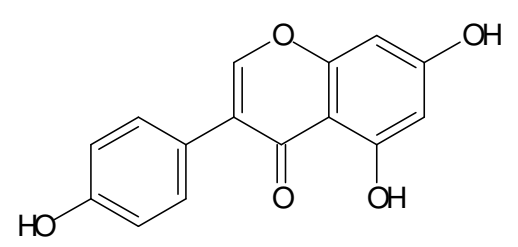

E1_12

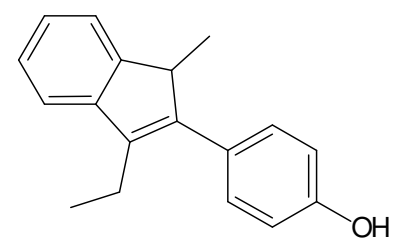

E1_13

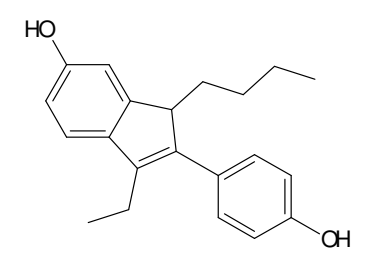

E1_14

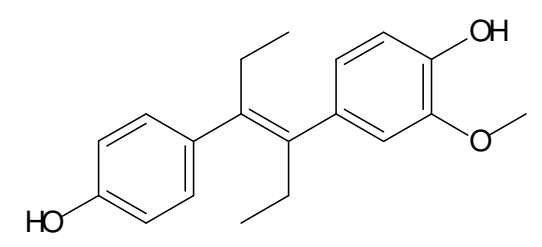

E1_15

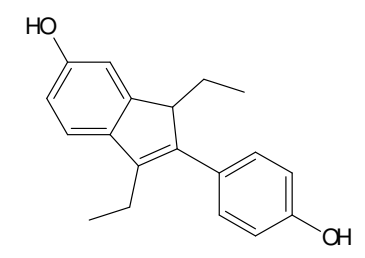

E1_16

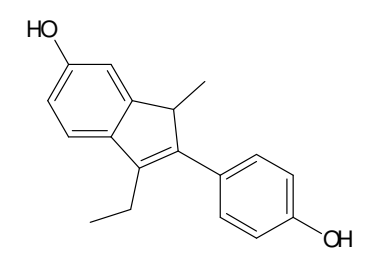

ERa

ERa

ERa

ERa

ERa

ERa

ERa

Sippl

Sippl

Sippl

Sippl

Sippl

Sippl

E1_17

ERa

Sippl 


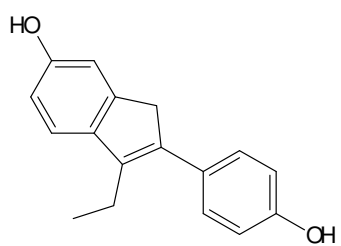

E1_18

ERa Sippl<smiles>CCCC1C(c2ccc(O)cc2)=C(CC)c2ccc(O)cc21</smiles>

E1_19<smiles>Oc1ccc(-c2ccccc2)cc1</smiles>

E1_2

ERa Sippl<smiles>CC/C(=C(\CC)c1ccc(OC)cc1)c1ccc(O)cc1</smiles>

E1_20

ERa Sippl<smiles>C/C=C(C(=C/C)\c1ccc(O)cc1)/c1ccc(O)cc1</smiles>

E1_21<smiles>CC/C(=C(/CC)c1ccc(O)c(O)c1)c1ccc(O)c(O)c1</smiles>

E1_22

ERa Sippl<smiles>CC(=C(C)c1ccc(O)cc1)c1ccc(O)cc1</smiles>

E1_23<smiles>CC12CCC3c4ccc(O)cc4CCC3C1CCC2O</smiles> 


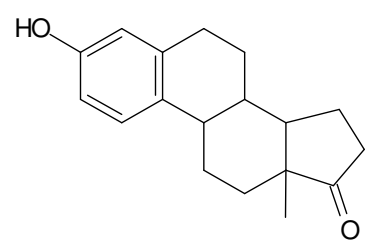

E1_25

ERa Sippl<smiles>O=c1oc2cc(O)ccc2c2oc3cc(O)ccc3c12</smiles>

E1_26<smiles>CC12CCC3c4ccc(O)cc4CCC3C1CCC2O</smiles>

E1_27

ERa Sippl<smiles>CCC1C(c2ccc(O)cc2)=C(C)c2cc(O)ccc21</smiles>

E1_28

ERa Sippl<smiles>CC/C(=C(\CC)c1ccc(O)c(O)c1)c1ccc(O)cc1</smiles>

E1_29

ERa Sippl<smiles>CC(C)(c1ccc(O)cc1)c1ccc(O)cc1</smiles>

E1_3<smiles>CCC1C(c2ccc(O)cc2)=C(C)c2cc(O)ccc21</smiles>

E1_30

ERa Sippl<smiles>CCC(c1ccc(O)cc1)C(CC)c1ccc(O)cc1</smiles> 


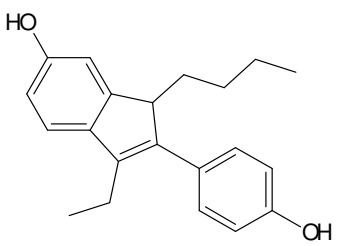

E1_32

ERa Sippl

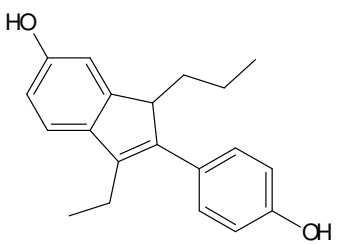

E1_33

ERa Sippl

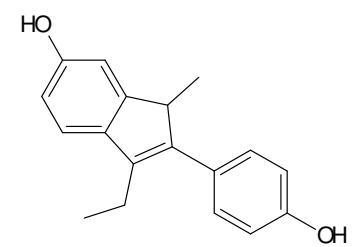

E1_34

ERa Sippl<smiles>CC/C(=C(\CC)c1ccc(O)cc1)c1ccc(O)cc1</smiles>

E1 35

ERa Sippl<smiles>CCC1=C(c2ccc(O)cc2)C(CC)c2cc(O)ccc21</smiles>

E1_36

ERa Sippl<smiles>CCC(=O)c1ccc(O)cc1</smiles>

E1_4

ERa Sippl<smiles>CCc1c(CC)c2ccc(O)cc2c2cc(O)ccc12</smiles>

E1_5

ERa Sippl<smiles>CCC1=C(c2ccccc2)C(C)c2cc(O)ccc21</smiles>

E1_6

ERa Sippl 
<smiles>C/C=C(C(=C/C)\c1ccc(O)cc1)/c1ccc(O)cc1</smiles>

E1_7

ERa Sippl<smiles>CC/C(=C(\CC)c1ccc(O)cc1)c1ccc(O)cc1</smiles>

E1_8

ERa Sippl

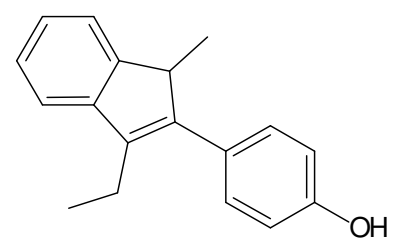

E1_9

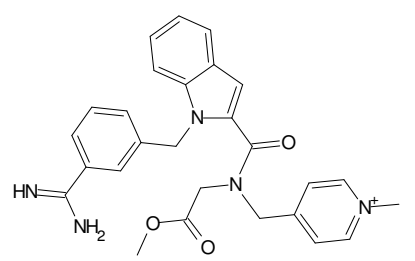

f1 1

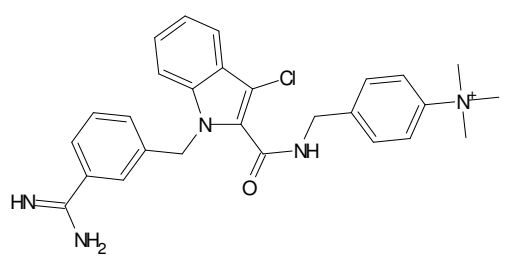

f1_10

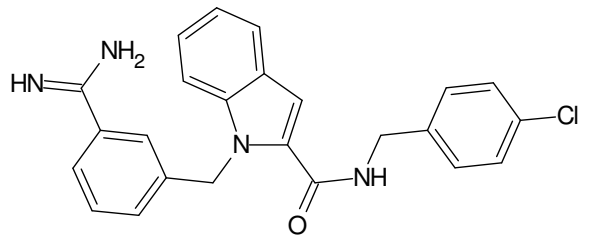

fXa Matter

f1_100

fXa Matter

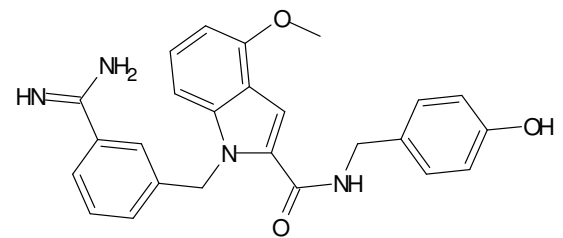

f1_101

fXa Matter

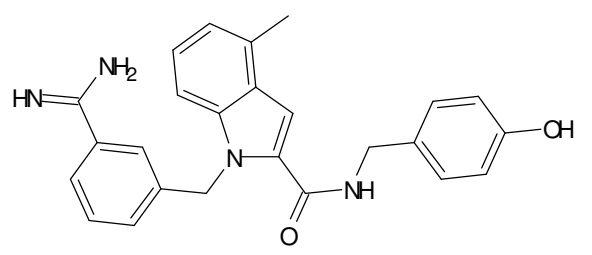


<smiles>COc1ccc(CNC(=O)c2cc3c(O)cccc3n2Cc2cccc(C(=N)N)c2)cc1</smiles>

f1_103

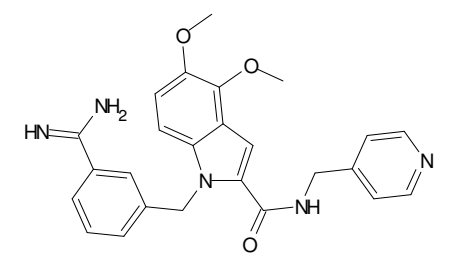

f1_104

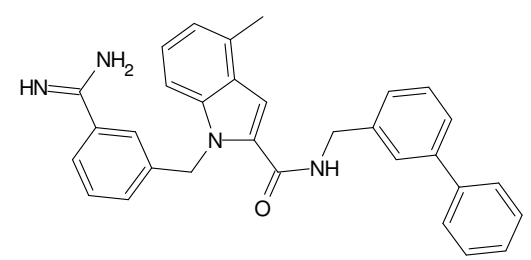

f1_106

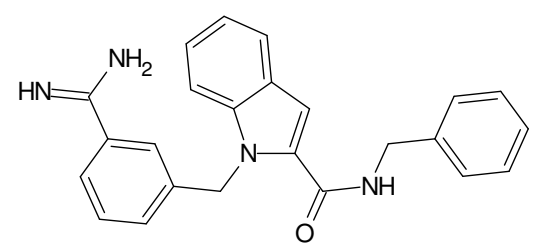

f1_107

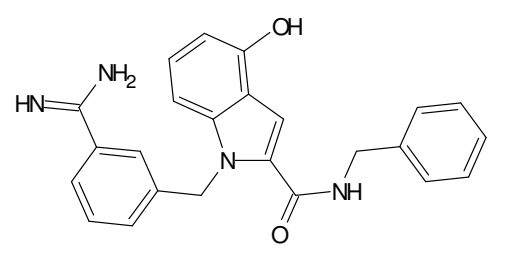

f1_108

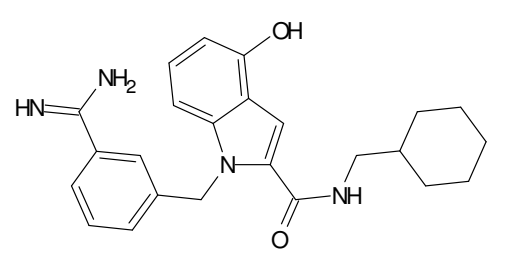

f1_109

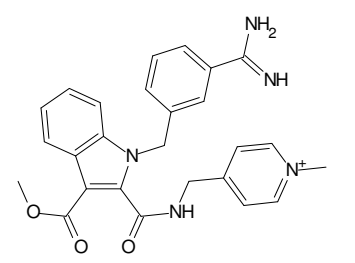

f1_11

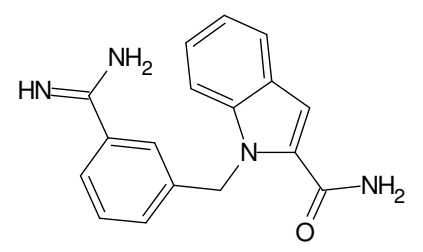

fXa Matter

fXa Matter

fXa Matter

fXa Matter

fXa Matter

fXa Matter

fXa Matter

fXa Matter 


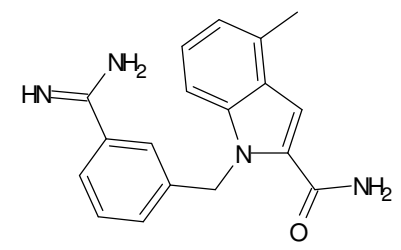

f1_111

fXa Matter

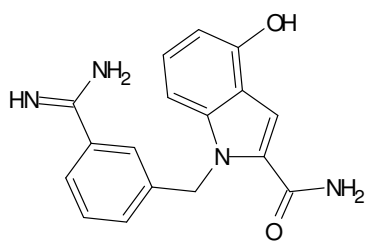

f1_ 112

fXa Matter

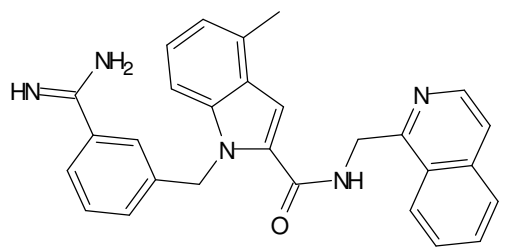

f1_ 113

fXa Matter

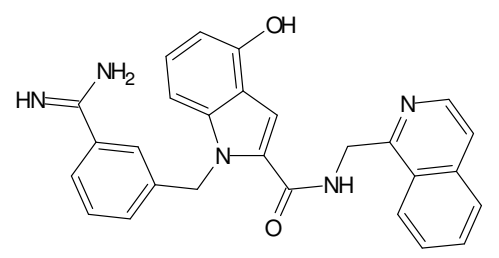

f1_ 114

fXa Matter

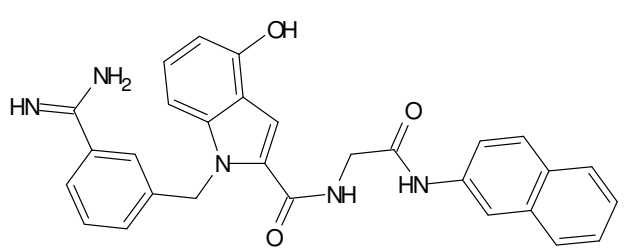

f1_ 115

fXa Matter

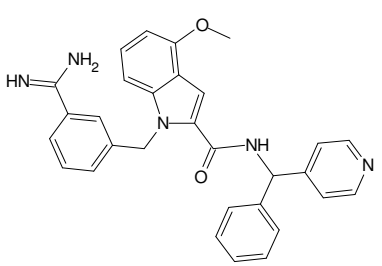

f1_116

fXa Matter

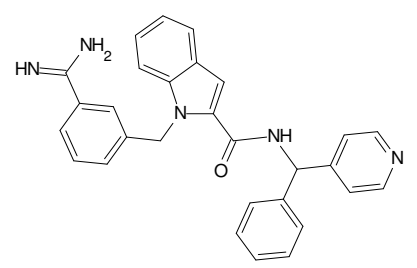

f1_ 117

fXa Matter<smiles>N=C(N)c1ccc(Cn2c(C(=O)NCc3cc4cccc5ccc6cccc3c4c56)cc3c(O)cccc32)cc1</smiles> 
<smiles>N=C(N)c1cccc(Cn2c(C(=O)NCc3ccccn3)cc3c(O)cccc32)c1</smiles>

f1_ 120

fXa Matter<smiles>N=C(N)c1cccc(Cn2c(C(=O)NCc3ccnc4ccccc34)cc3c(O)cccc32)c1</smiles>

f1_ 121<smiles>Cc1cccc2c1cc(C(=O)NCc1ccnc3ccccc13)n2Cc1cccc(C(=N)N)c1</smiles>

f1_122

fXa Matter<smiles>COC(=O)c1c(C)c2ccccc2n1Cc1cccc(C(=N)N)c1</smiles>

f1_ 123<smiles>COC(=O)c1c(C(=O)OC)n(Cc2cccc(C(=N)N)c2)c2ccccc12</smiles>

f1_124

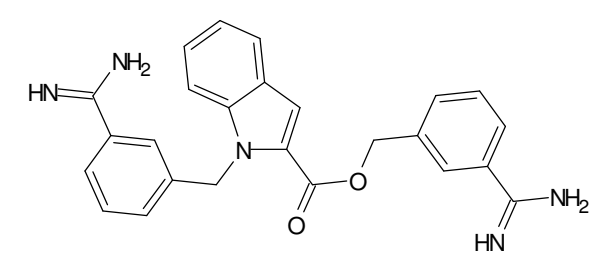

fXa Matter

f1_ 125

fXa Matter

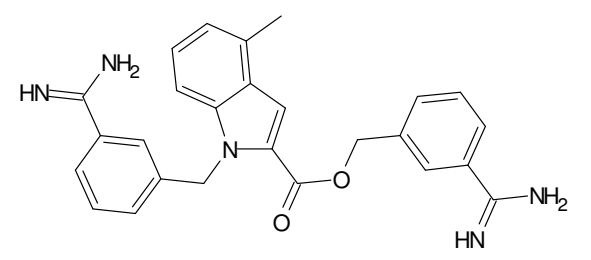

f1_126

fXa Matter<smiles>CCOC(=O)c1cc2ccccc2n1Cc1ccc(C(=N)N)cc1</smiles> 


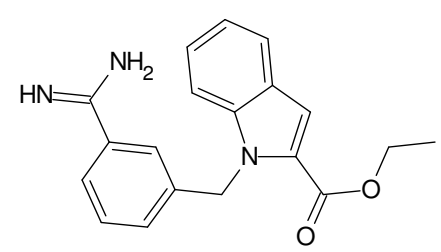

f1_128

fXa Matter

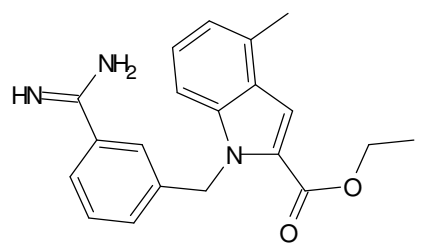

f1_129

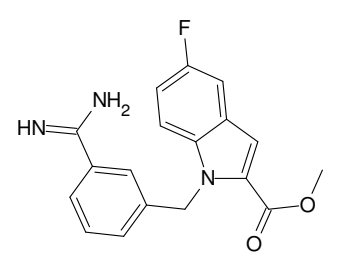

fXa Matter

fXa Matter

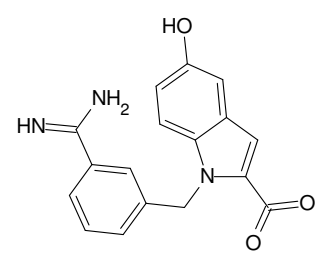

f1_131

fXa Matter<smiles>CSC(=O)c1cc2c(O)cccc2n1Cc1cccc(C(=N)N)c1</smiles>

f1_132

fXa Matter

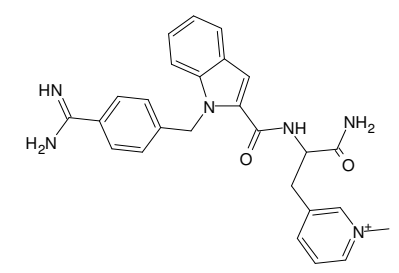

f1_133

fXa Matter

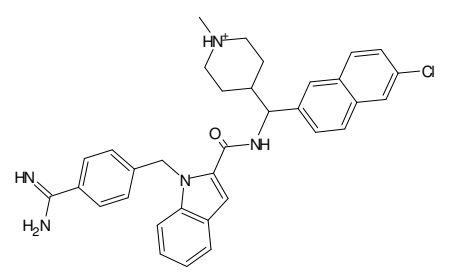

f1_134

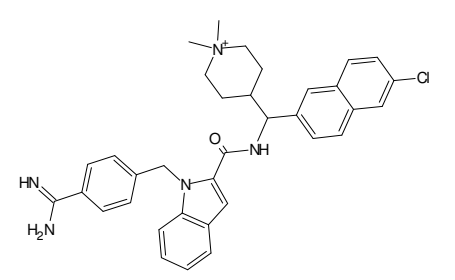

fXa Matter

f1_135 
f1 136
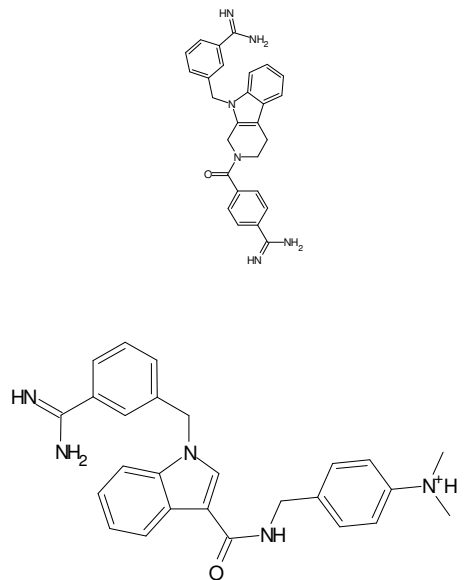

f1_137

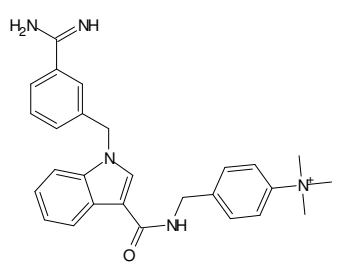

f1_138

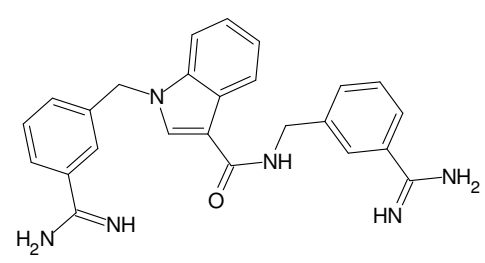

f1_139

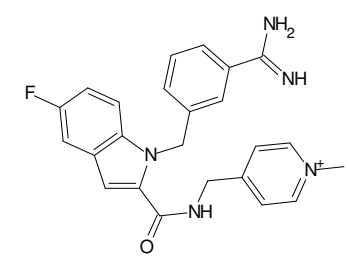

f1_14

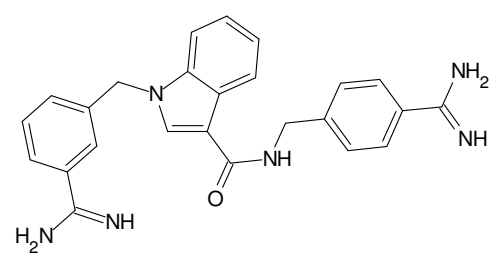

f1_140

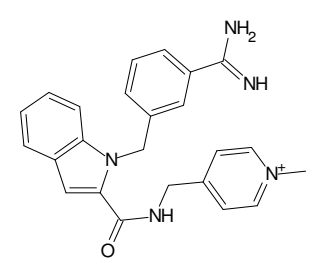

f1_15

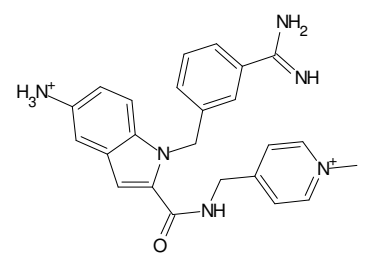

fXa Matter

fXa Matter

fXa Matter

fXa Matter

fXa Matter

fXa Matter

fXa Matter

fXa Matter 
f1_17

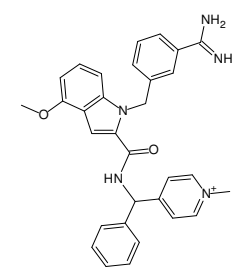

-

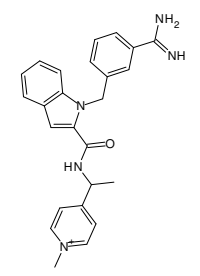

f1_18

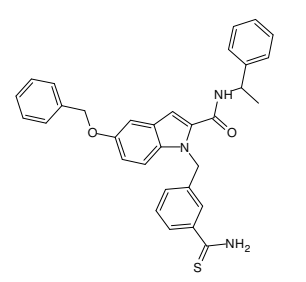

f1_19

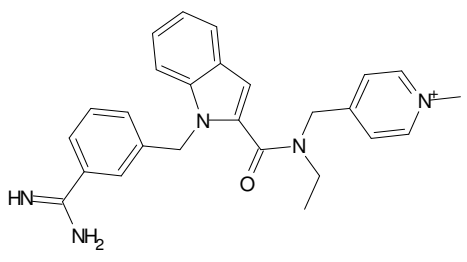

f1_2

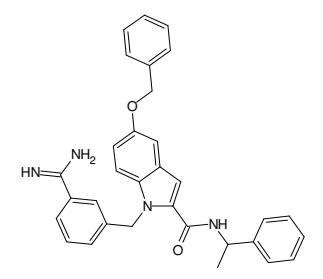

f1_20

fXa Matter

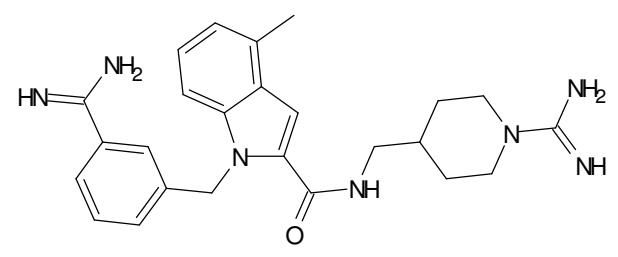

f1_21

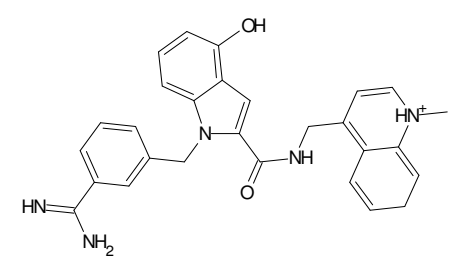

f1_22

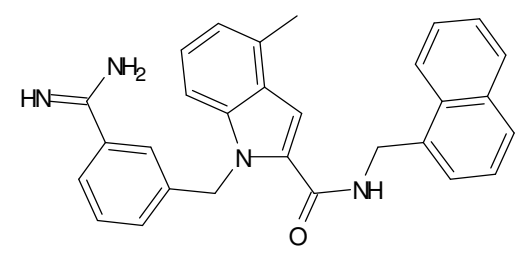

f1_23 


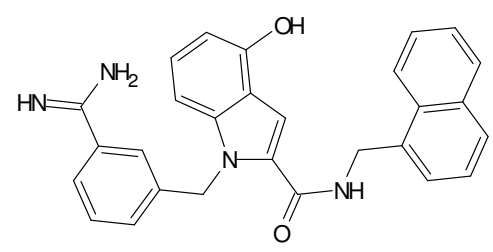

f1_24

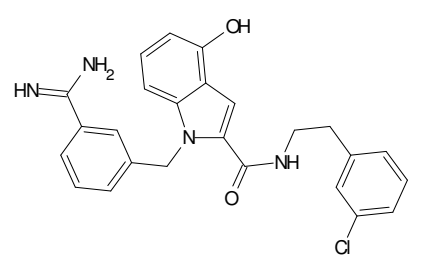

fXa Matter

f1_27

fXa Matter

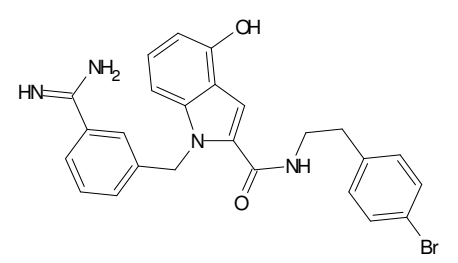

f1_28

fXa Matter

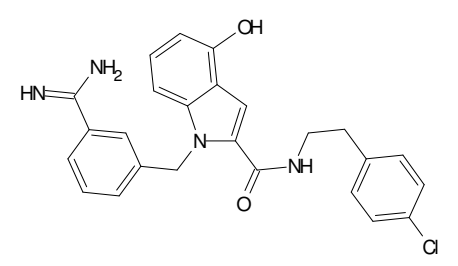

f1_29

fXa Matter

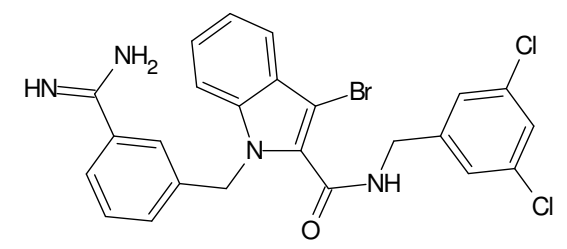

f1_3

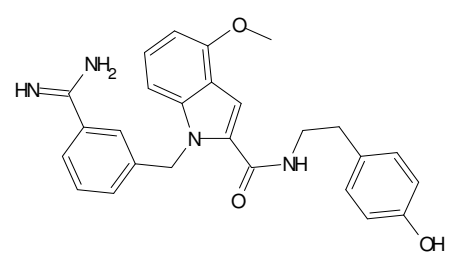

f1_30

fXa Matter

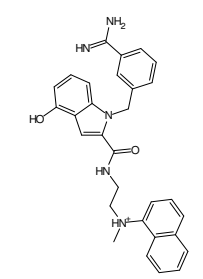

f1_31

fXa Matter

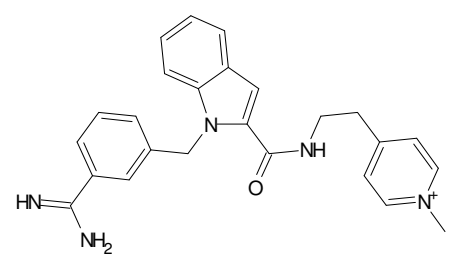

fXa Matter 


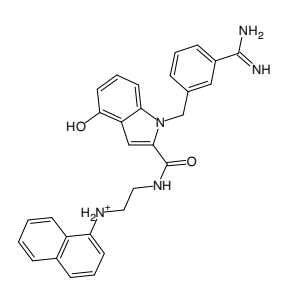

f1_33

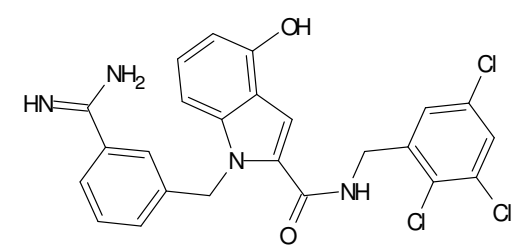

f1_34

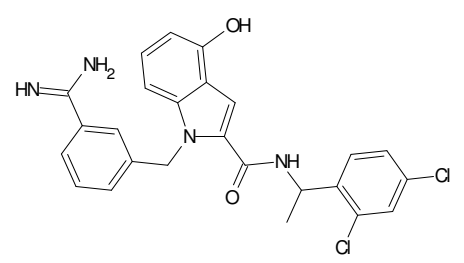

f1_35

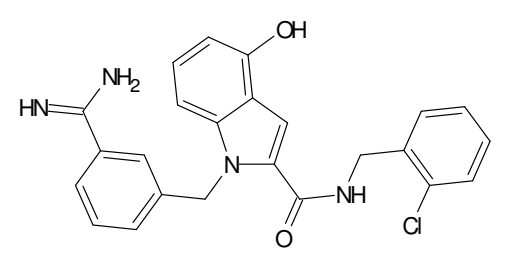

f1_36

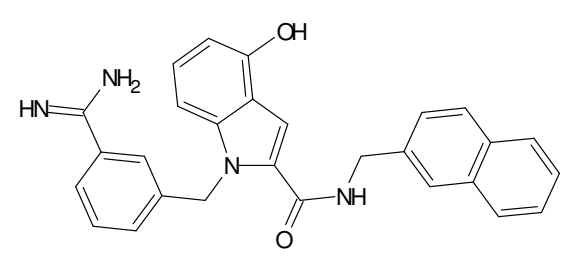

f1_37

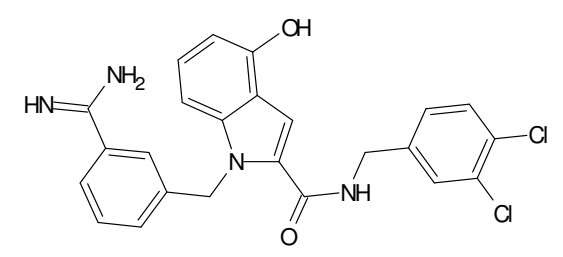

f1_38

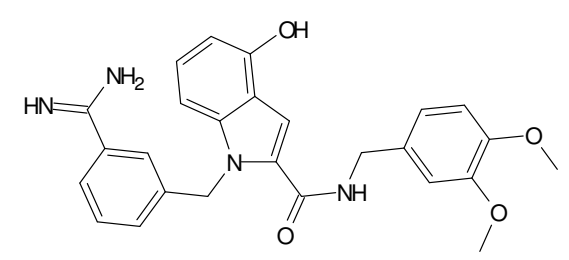

f1_39

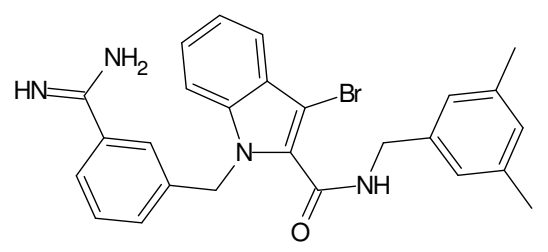

fXa Matter

fXa Matter

fXa Matter

fXa Matter

fXa Matter

fXa Matter

fXa Matter

fXa Matter 


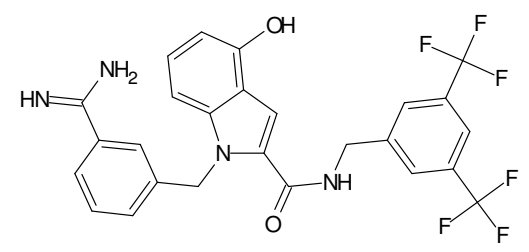

f1_40

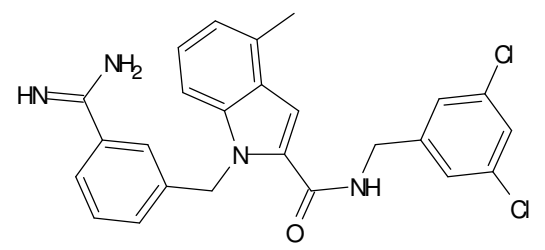

f1_41

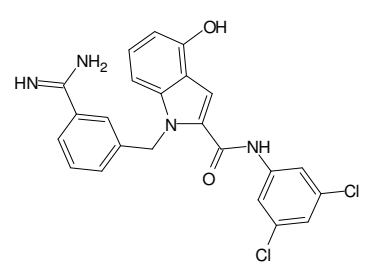

fXa Matter

f1_42

fXa Matter

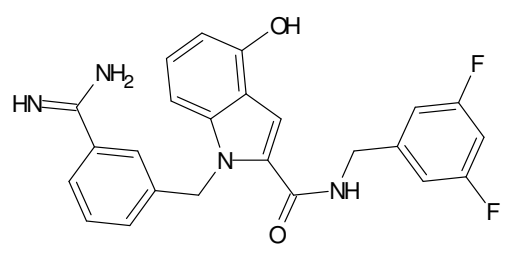

f1_43

fXa Matter

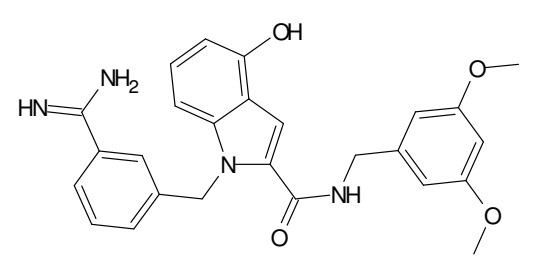

f1_44

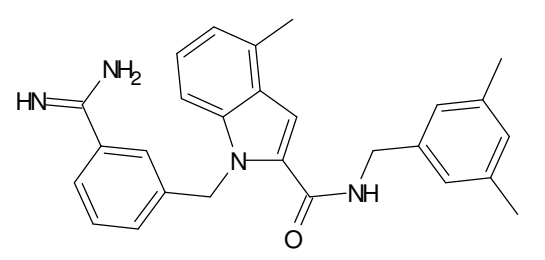

f1_45

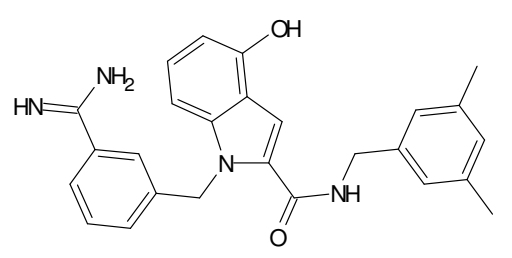

fXa Matter

f1_46

fXa Matter<smiles>Cc1cccc2c1cc(C(=O)NCc1cccc(C(=N)N)c1)n2Cc1cccc(C(=N)N)c1</smiles> 


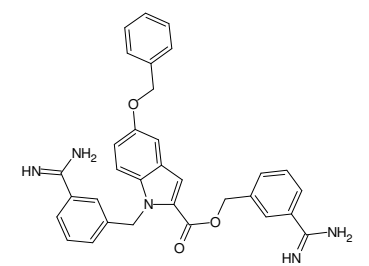

f1_ 48

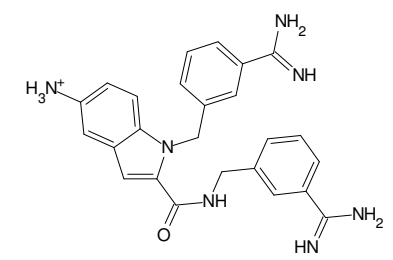

f1_ 49

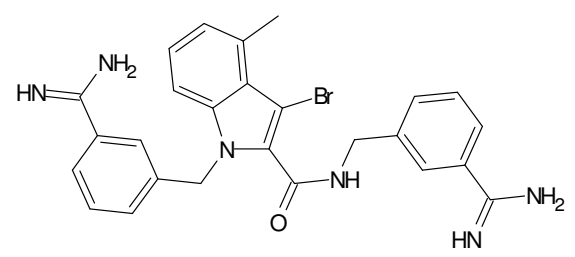

f1_5

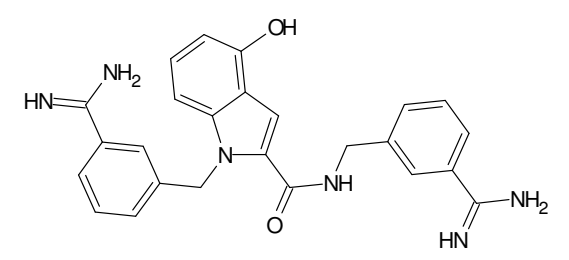

f1_50

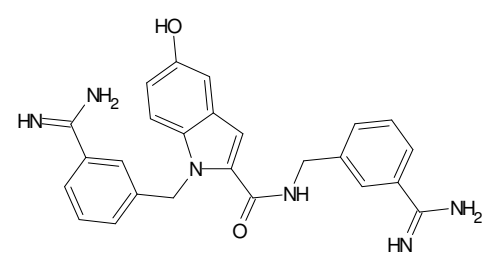

f1_51

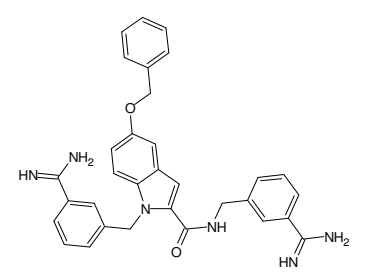

f1_52

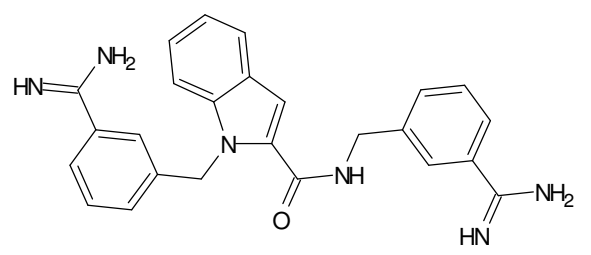

f1_53

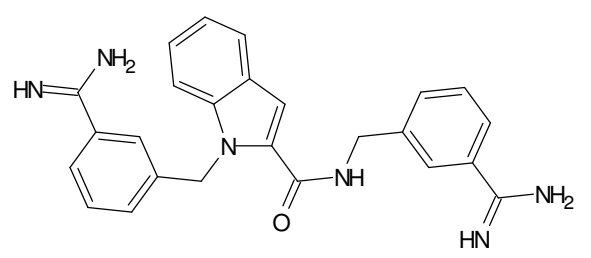

fXa Matter

fXa Matter

fXa Matter

fXa Matter

fXa Matter

fXa Matter

fXa Matter

$$
\text { fXa Matter }
$$




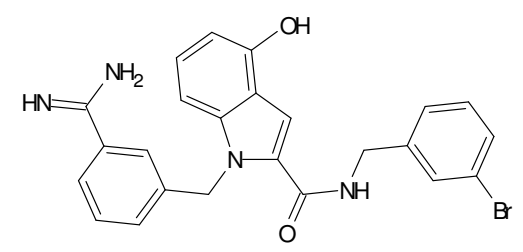

f1_55

fXa Matter

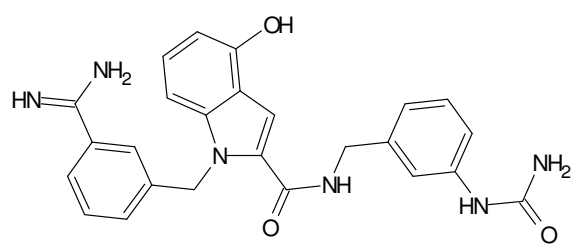

f1_56

fXa Matter

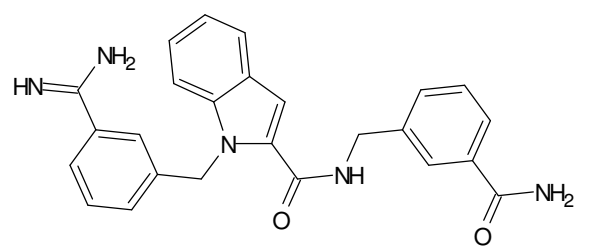

f1_57

fXa Matter

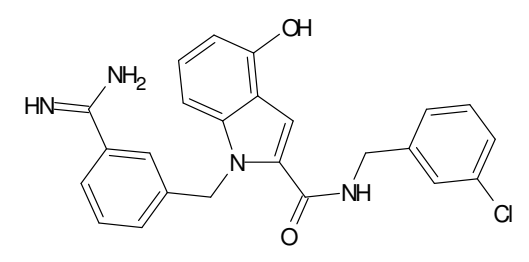

f1_58

fXa Matter

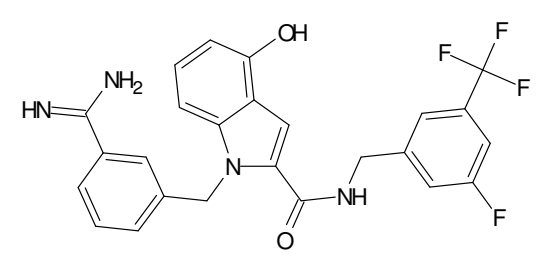

f1_59

fXa Matter

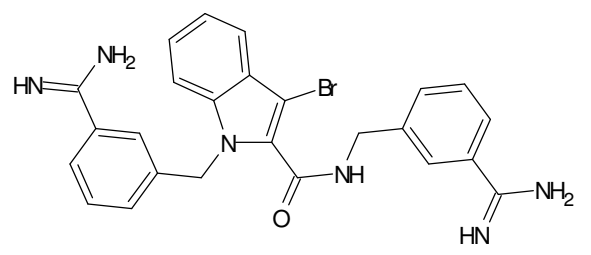

f1_6

fXa Matter

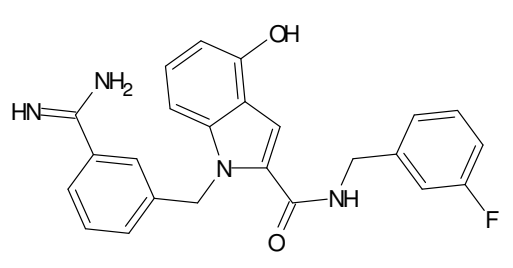

f1_60

fXa Matter

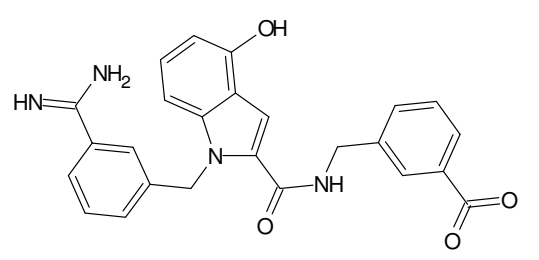




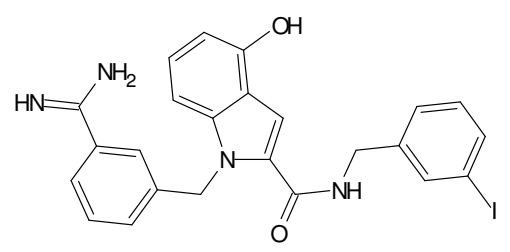

f1_62

fXa Matter

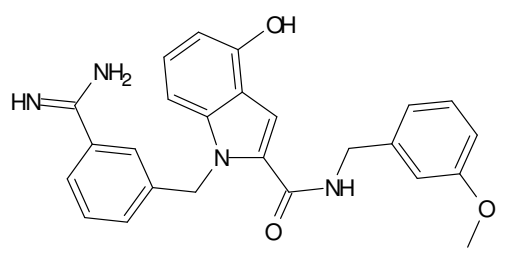

f1_63

fXa Matter

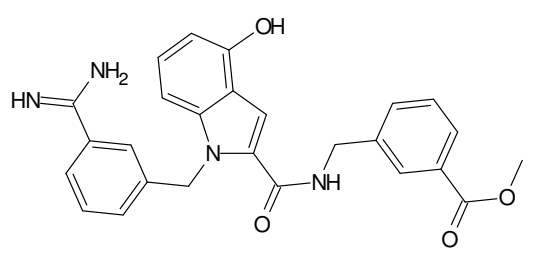

f1_64

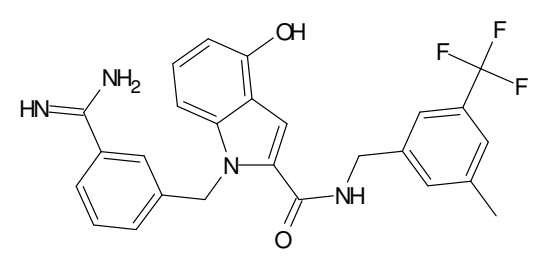

fXa Matter

f1_65

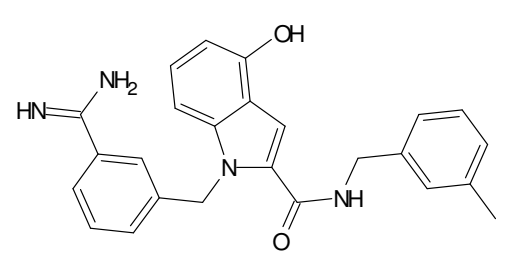

fXa Matter

f1_66

fXa Matter

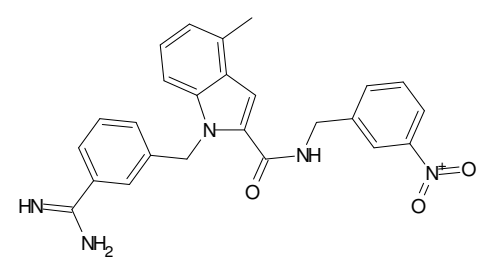

f1_67

fXa Matter

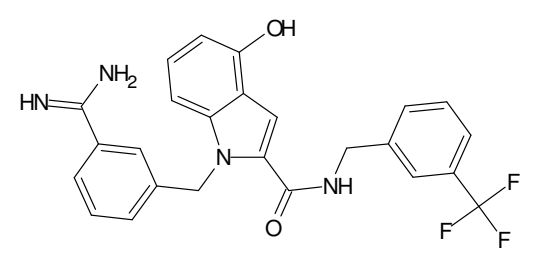

f1_68

fXa Matter

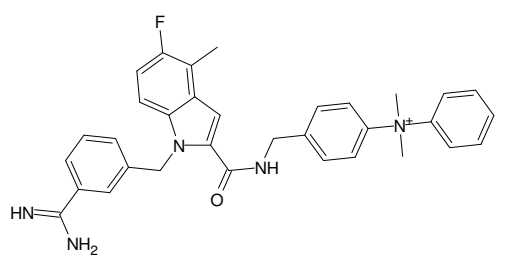

f1_69 


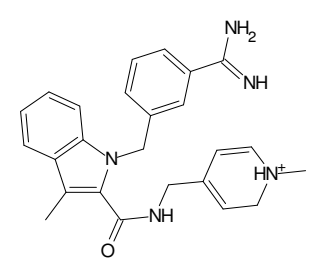

f1_7

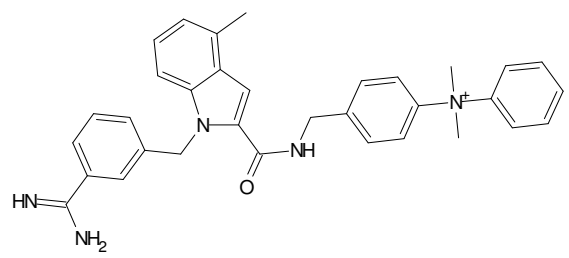

f1_70

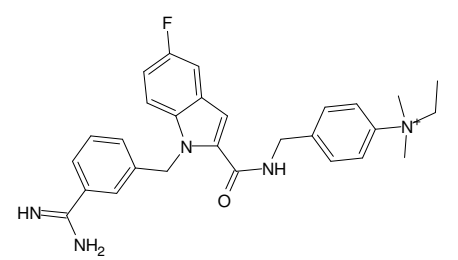

f1_73

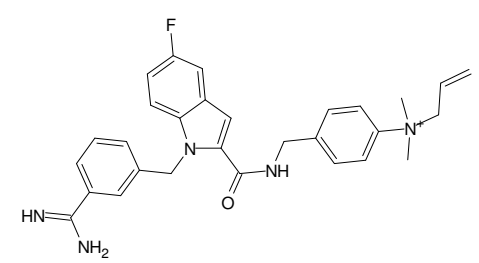

f1_74

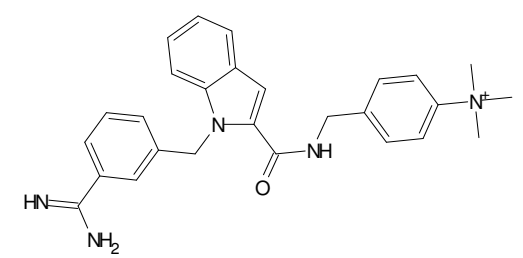

f1_76

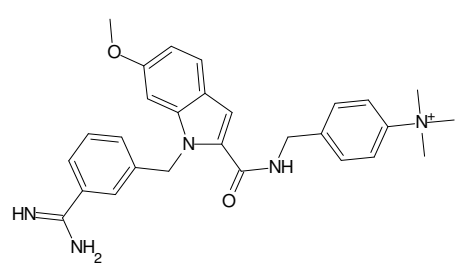

f1_77

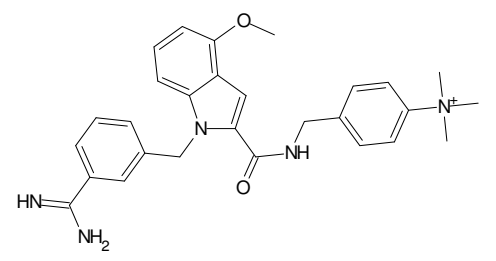

f1_78

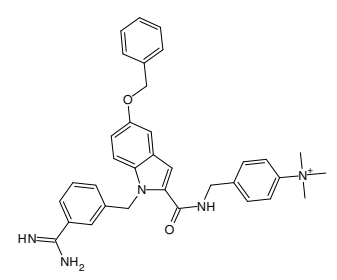

fXa Matter

fXa Matter

fXa Matter

fXa Matter

fXa Matter

fXa Matter

fXa Matter

fXa Matter 


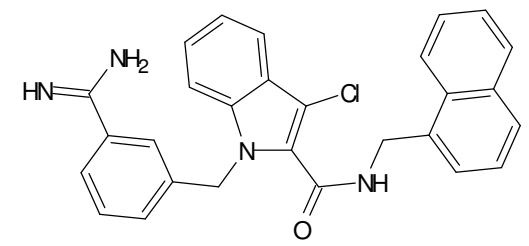

f1_8

fXa Matter

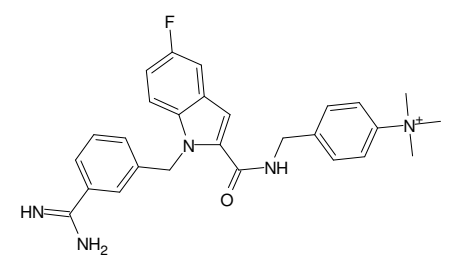

f1_80

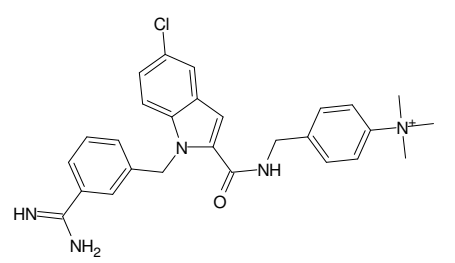

f1_81

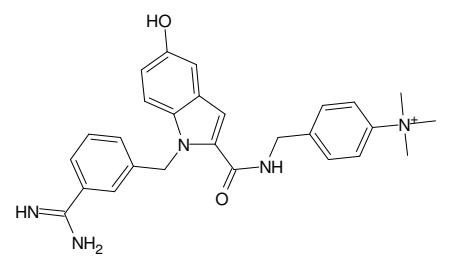

f1_82

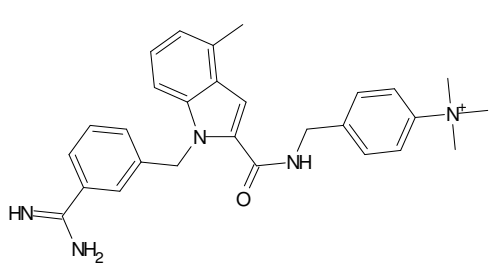

f1_83

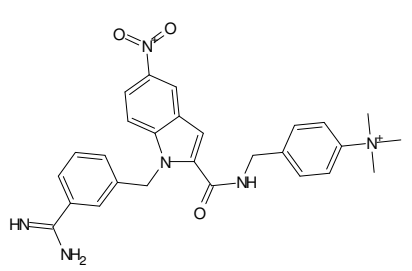

f1_84

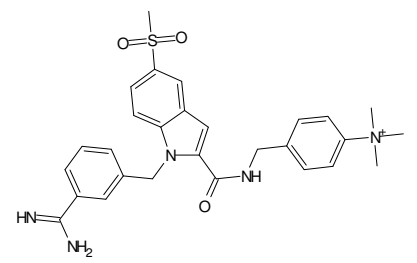

f1_86

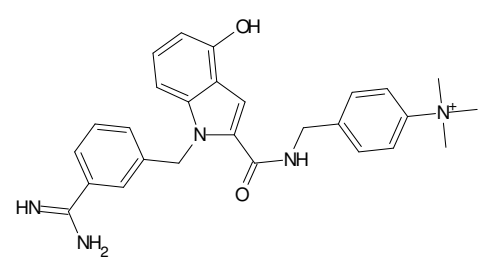

fXa Matter

fXa Matter

fXa Matter 


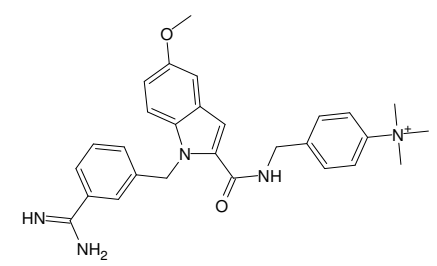

f1_88

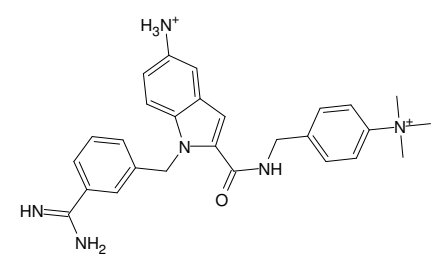

f1_89

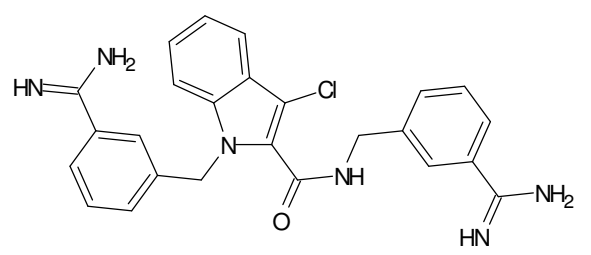

fXa Matter

f1_9

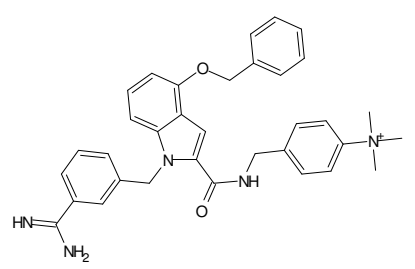

f1_90

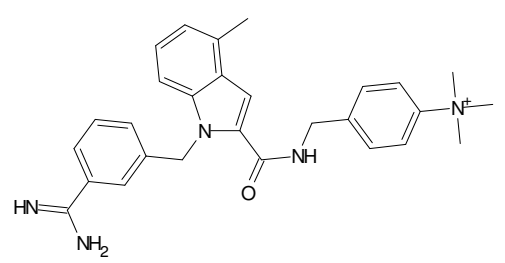

f1_91

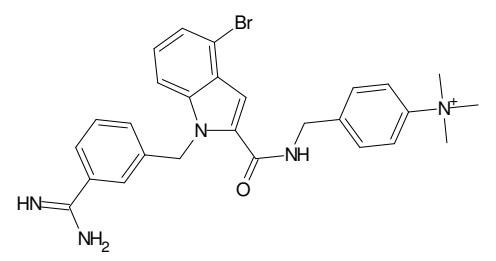

f1_92

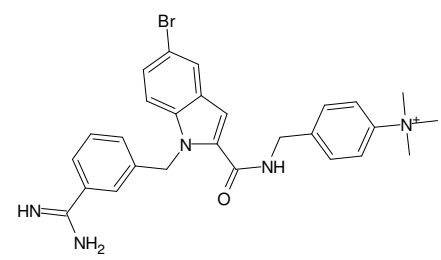

f1_93

fXa Matter

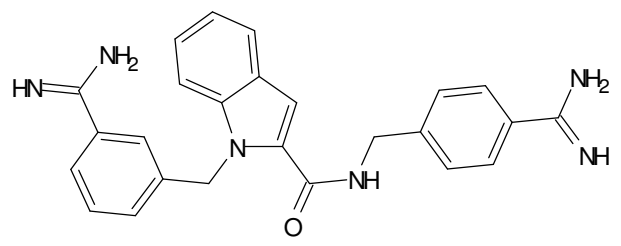

f1_95

fXa Matter 


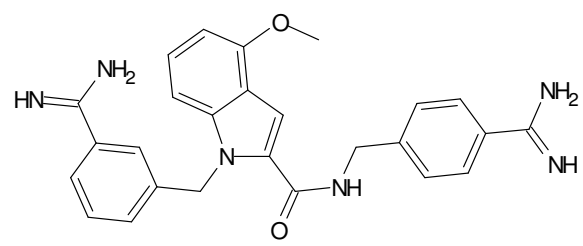

f1_96

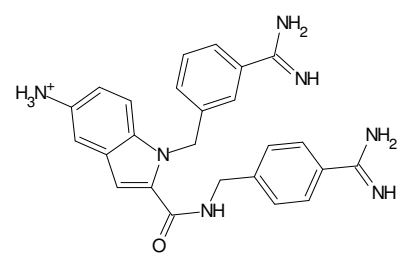

f1_97

fXa Matter

fXa Matter

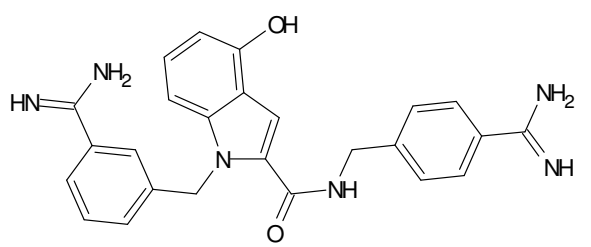

f1_98

fXa Matter

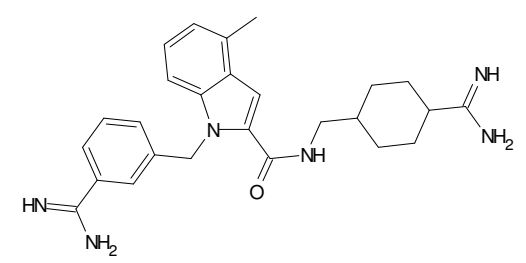

f1_99

fXa Matter

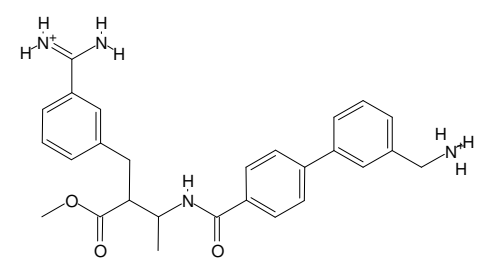

1ezq_lig

fXa PDB

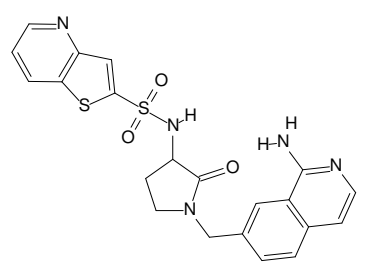

1fOr_lig

fXa PDB

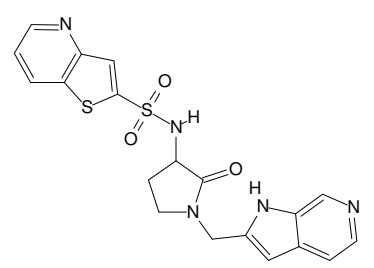

1f0s_lig

fXa PDB

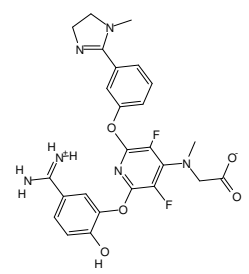

1fjs_lig 


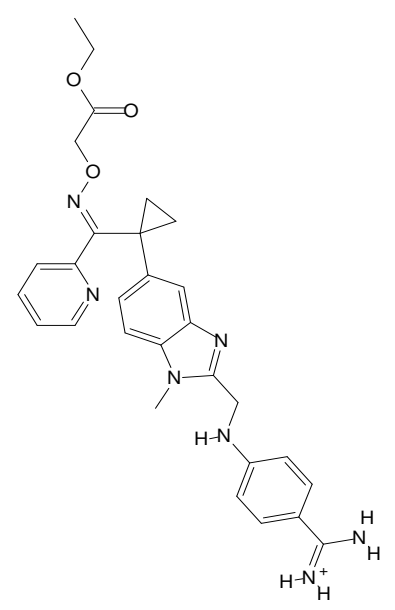

1g2l_lig

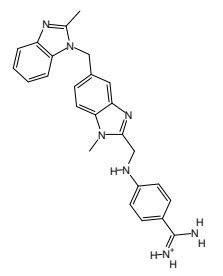

1g2m_lig

fXa PDB

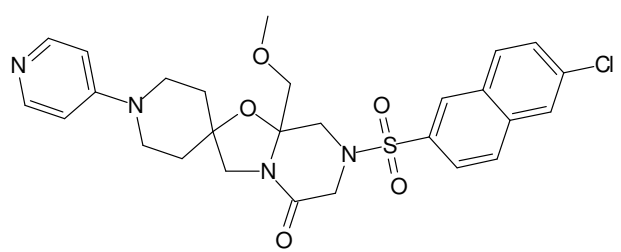

1ioe_lig

fXa PDB

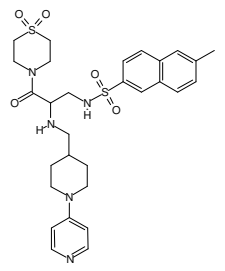

1iqe_lig

$f X a \quad$ PDB

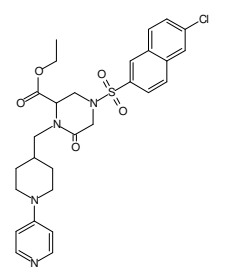

1iqf_lig

$f X a \quad$ PDB

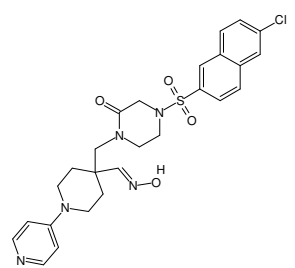

1iqg_lig

$\mathrm{fXa} \quad$ PDB

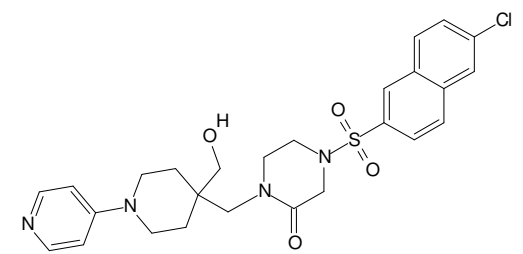

1iqh_lig

fXa PDB 


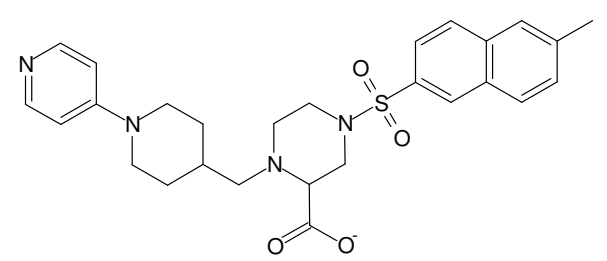

1iqi_lig

fXa PDB

1iqj_lig

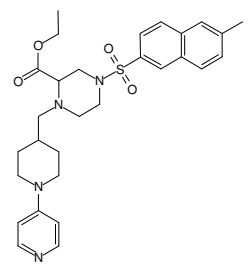

fXa PDB

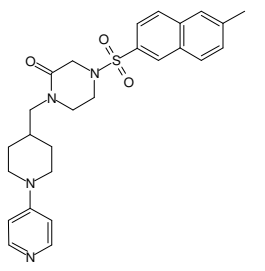

1iqk_lig

fXa PDB

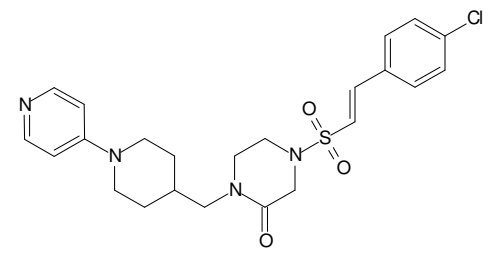

1iql_lig

fXa PDB

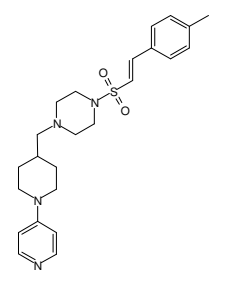

1iqm_lig

fXa PDB

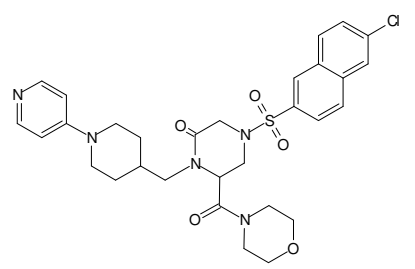

1iqn_lig

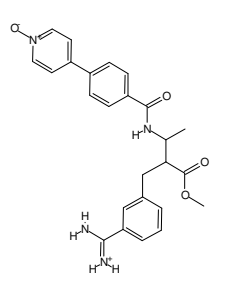

1ksn_lig

fXa PDB

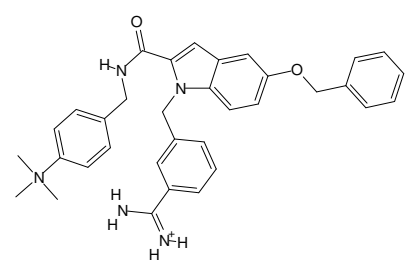

fXa PDB

1lpg_lig

fXa PDB 


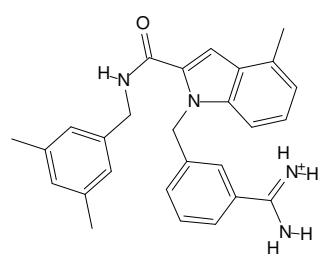

1lqd_lig

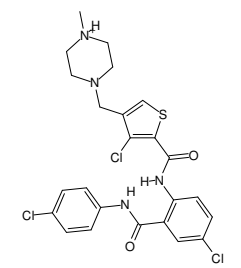

1mq5_lig

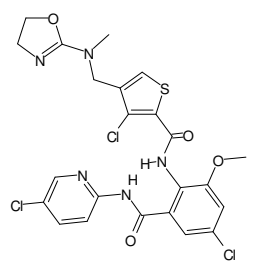

1mq6_lig

1nfu_lig
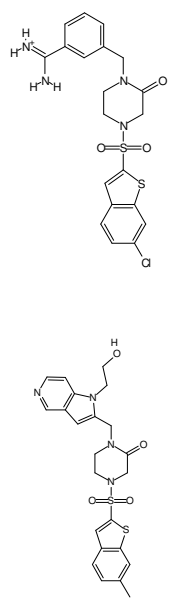

1nfx_lig

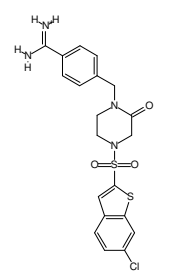

1nfy_lig

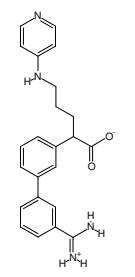

1xka_lig

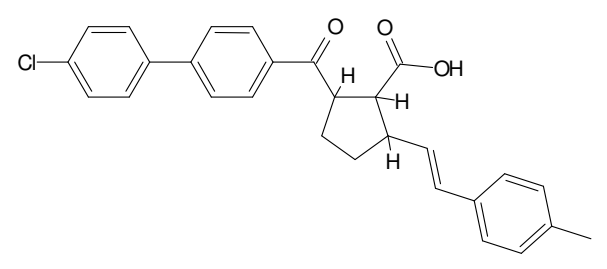

fXa PDB

fXa PDB

fXa PDB

fXa PDB

fXa PDB

fXa PDB

fXa PDB

MMP3 $\mathrm{Ha}$ 


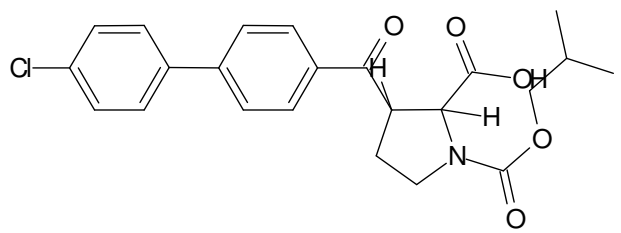

M1_10

MMP3 $\mathrm{Ha}$

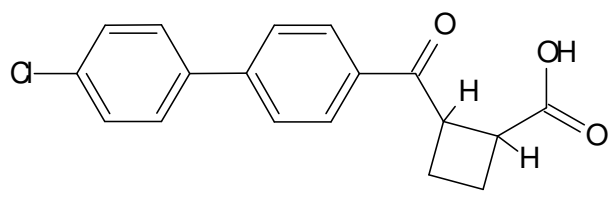

M1_11

MMP3 $\mathrm{Ha}$

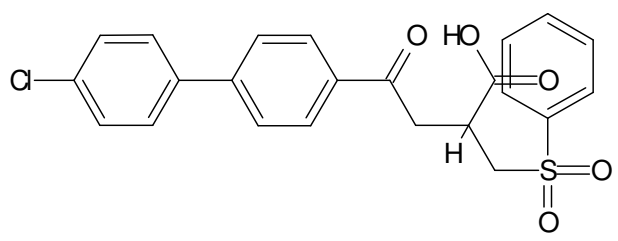

M1_12

MMP3 $\mathrm{Ha}$

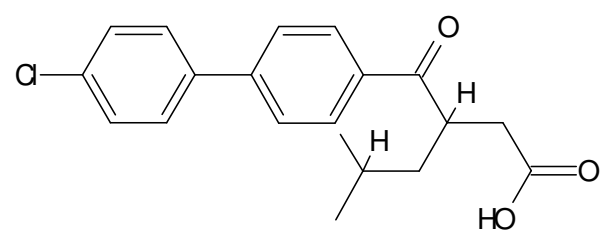

M1_13

MMP3 $\mathrm{Ha}$

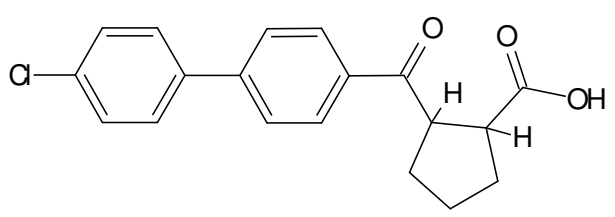

M1_14

MMP3 $\mathrm{Ha}$

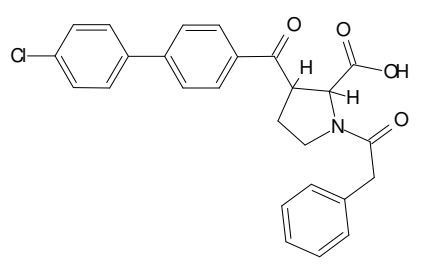

M1_15

MMP3 $\mathrm{Ha}$

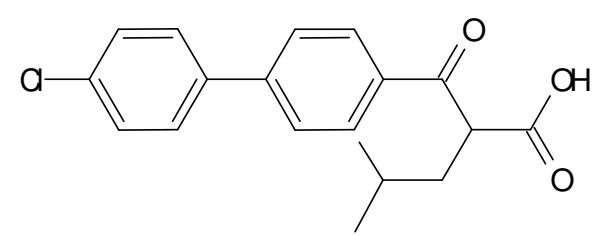

M1_16

MMP3 $\mathrm{Ha}$

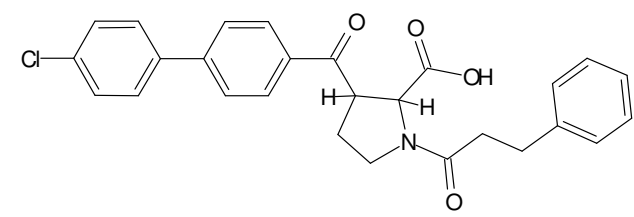

M1_17

MMP3 $\mathrm{Ha}$ 


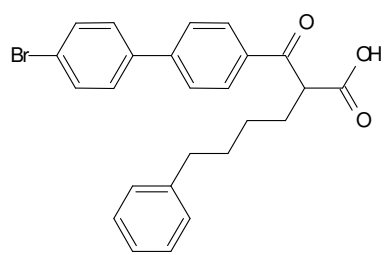

M1 18

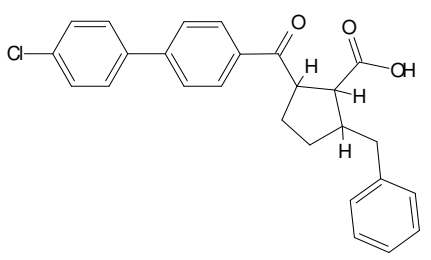

M1_19

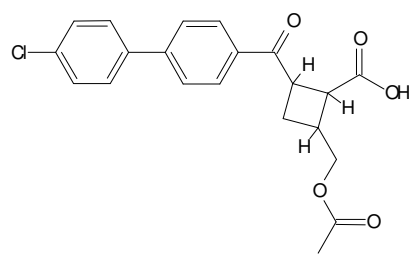

M1_2

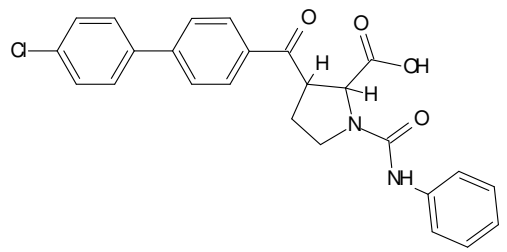

M1_20

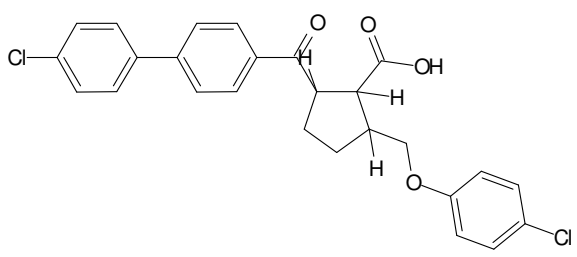

M1_21

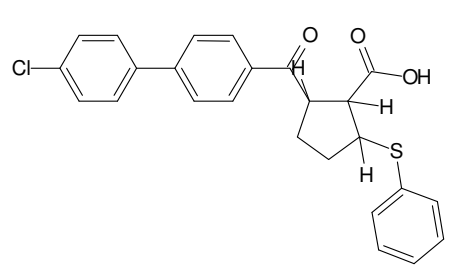

M1_22

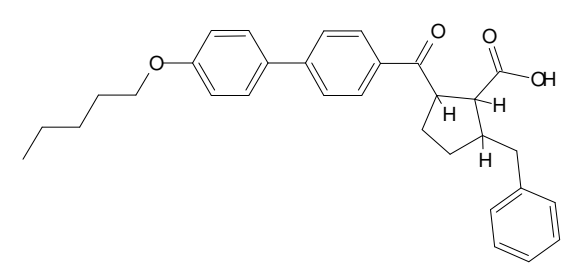

M1_23

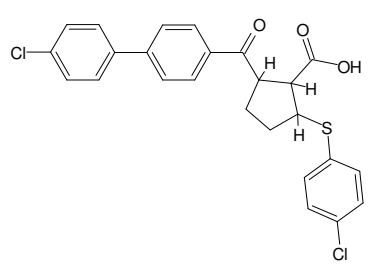

MMP3 $\mathrm{Ha}$

MMP3 $\mathrm{Ha}$

MMP3 $\mathrm{Ha}$

MMP3 $\mathrm{Ha}$

MMP3 $\mathrm{Ha}$

MMP3 $\mathrm{Ha}$

MMP3 $\mathrm{Ha}$

MMP3 $\mathrm{Ha}$ 


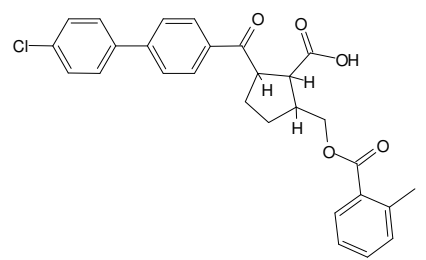

M1_25

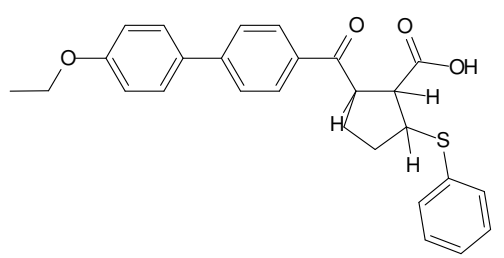

M1_26

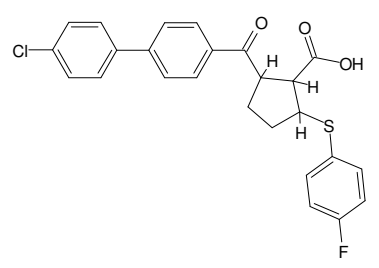

M1_27

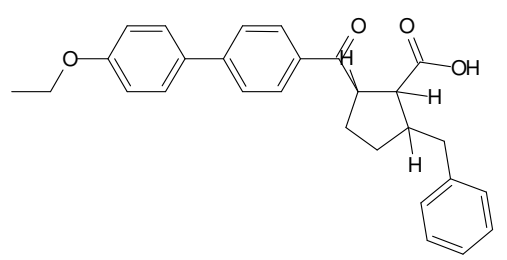

M1_28

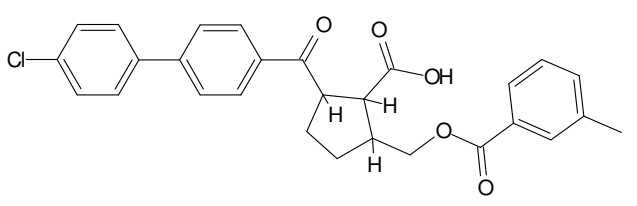

M1_29

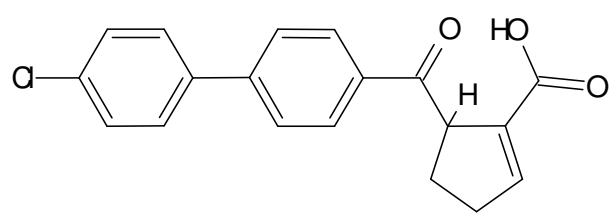

M1_3

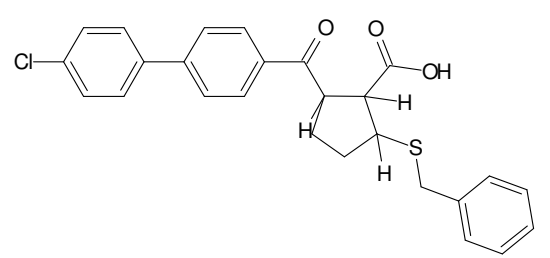

M1_30

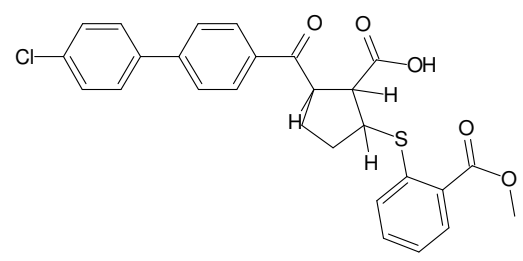

M1 31
MMP3 $\mathrm{Ha}$

MMP3 $\mathrm{Ha}$

MMP3 $\mathrm{Ha}$

MMP3 $\mathrm{Ha}$

MMP3 $\mathrm{Ha}$

MMP3 $\mathrm{Ha}$

MMP3 Ha

MMP3 $\mathrm{Ha}$ 


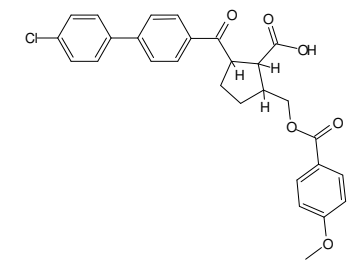

M1_32

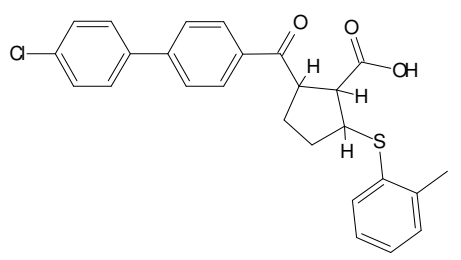

M1_33

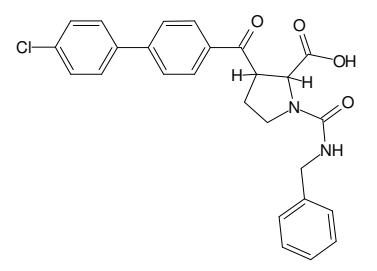

M1_34

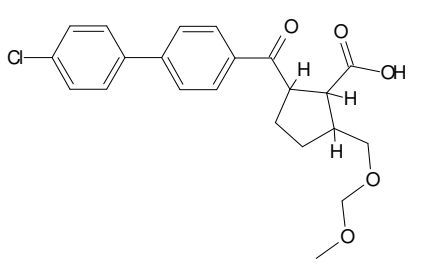

M1_35

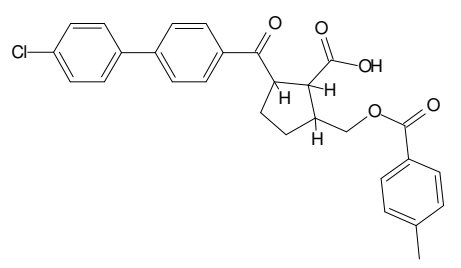

M1_36

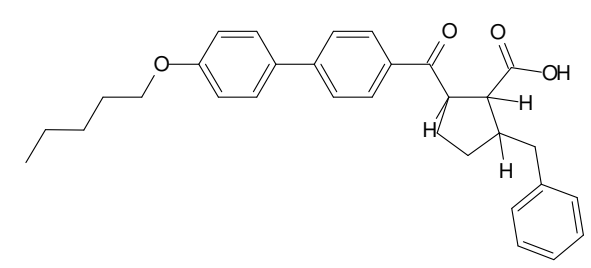

M1_37

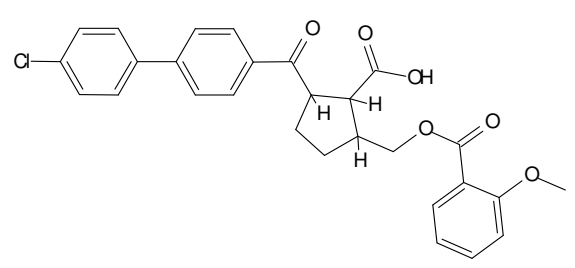

M1_38

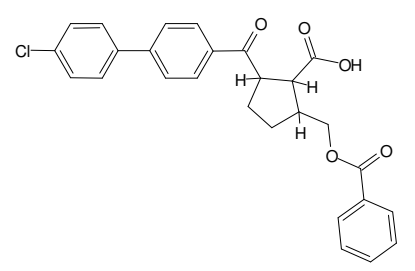

MMP3 Ha

MMP3 $\mathrm{Ha}$

MMP3 $\mathrm{Ha}$

MMP3 $\mathrm{Ha}$

MMP3 $\mathrm{Ha}$

MMP3 $\mathrm{Ha}$

MMP3 $\mathrm{Ha}$

MMP3 $\mathrm{Ha}$ 


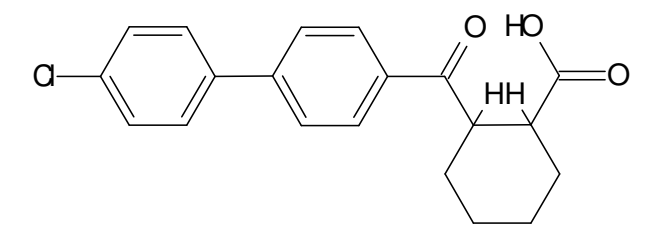

M1_4

MMP3 $\mathrm{Ha}$

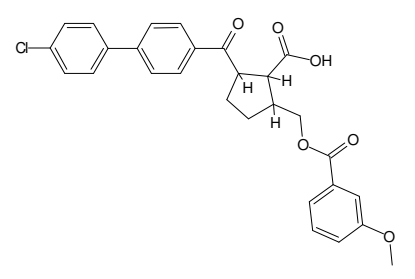

M1_40

MMP3 $\mathrm{Ha}$

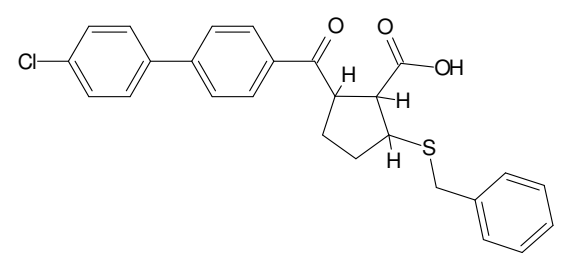

M1_41

MMP3 $\mathrm{Ha}$

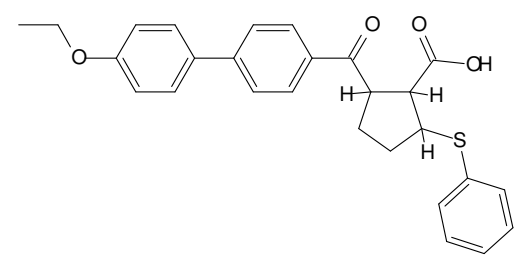

M1_42

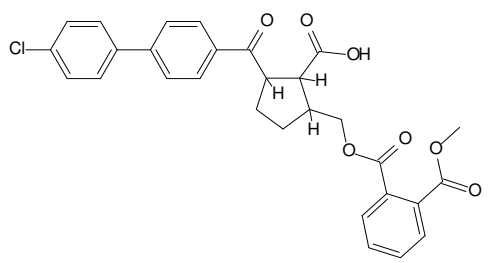

M1_43

MMP3 $\mathrm{Ha}$

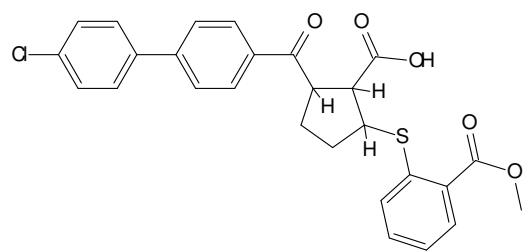

M1_44

MMP3 $\mathrm{Ha}$

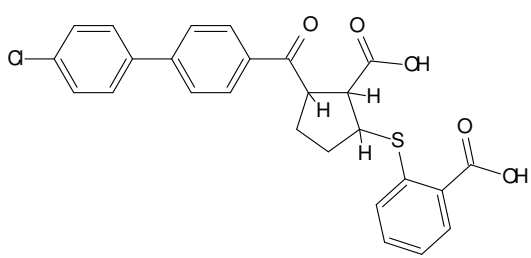

M1_45

MMP3 Ha

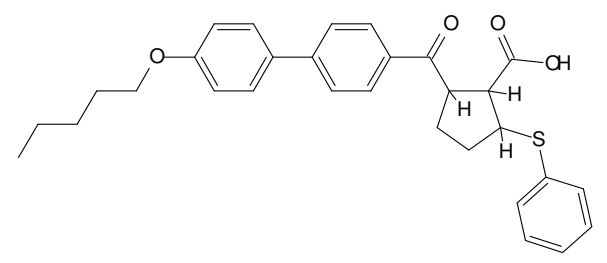

M1_46

MMP3 Ha 


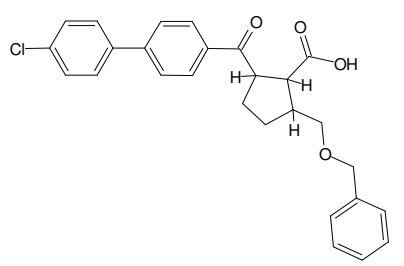

M1_47

MMP3 $\mathrm{Ha}$

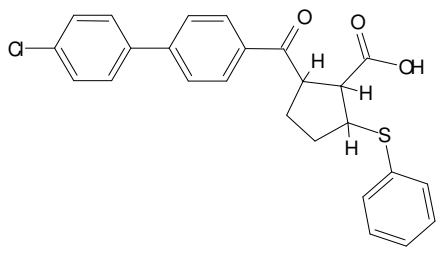

M1_48

MMP3 $\mathrm{Ha}$

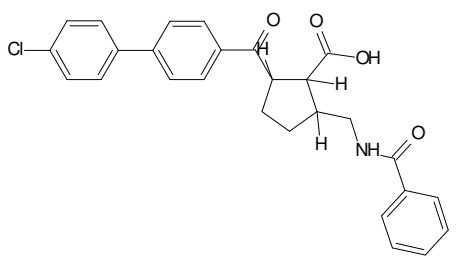

M1_49

MMP3 $\mathrm{Ha}$

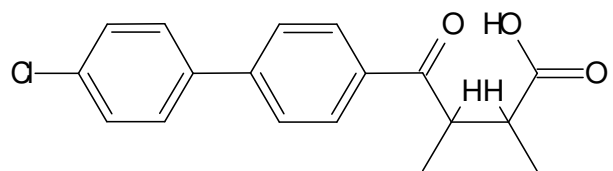

M1_5

MMP3 $\mathrm{Ha}$

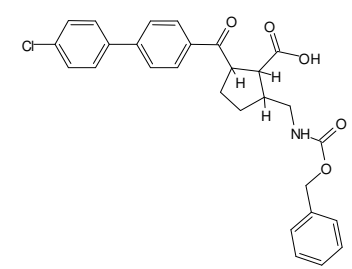

M1_50

MMP3 $\mathrm{Ha}$

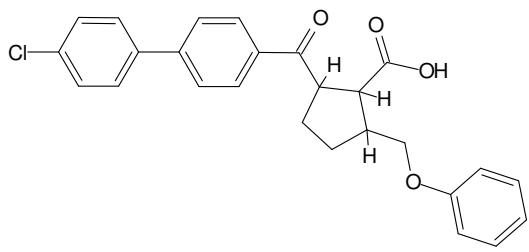

M1_51

MMP3 $\mathrm{Ha}$

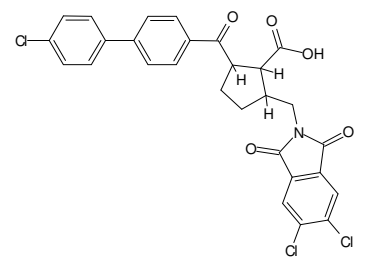

M1_52

MMP3 $\mathrm{Ha}$

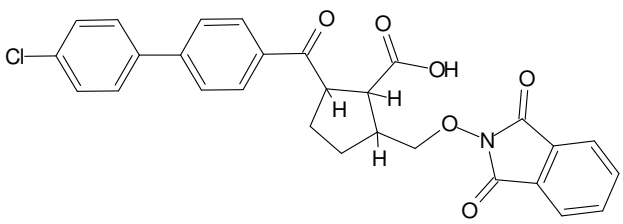

M1_53

MMP3 $\mathrm{Ha}$ 


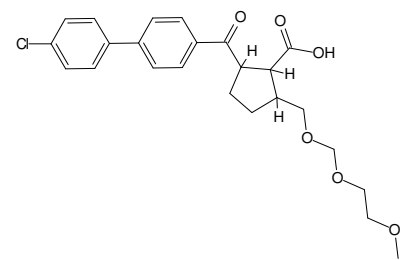

M1 54

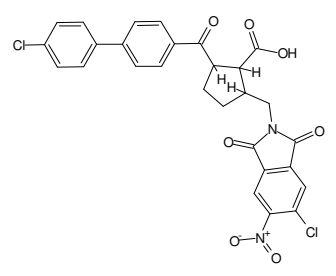

M1_55

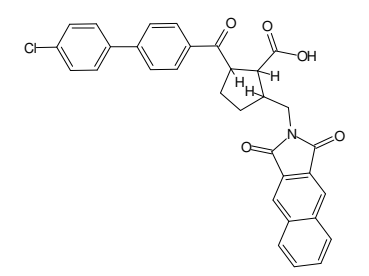

M1_56

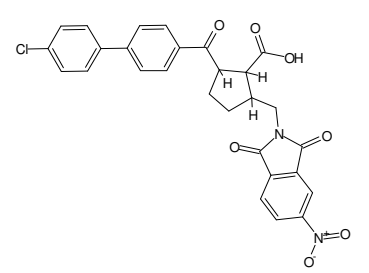

M1_57

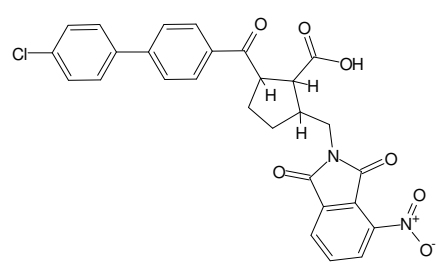

M1_58

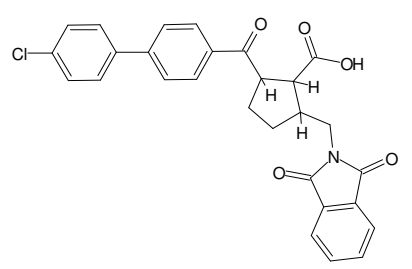

M1_59

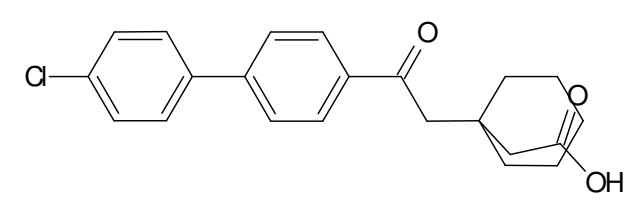

M1_6

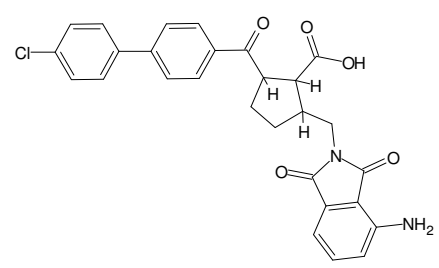

M1_60
MMP3 $\mathrm{Ha}$

MMP3 $\mathrm{Ha}$

MMP3 $\mathrm{Ha}$

MMP3 $\mathrm{Ha}$

MMP3 $\mathrm{Ha}$

MMP3 $\mathrm{Ha}$

MMP3 Ha

MMP3 $\mathrm{Ha}$ 


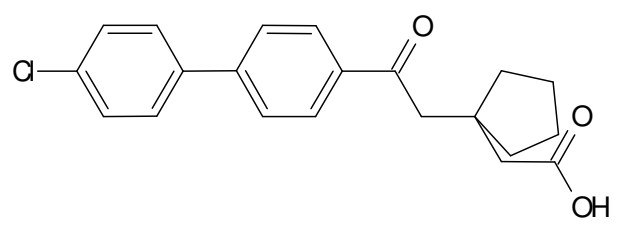

M1_7

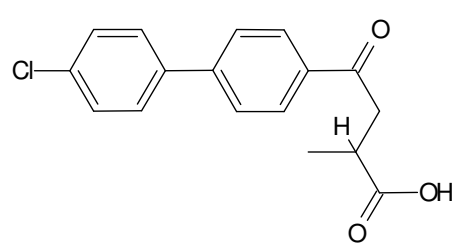

M1_8

MMP3 $\mathrm{Ha}$

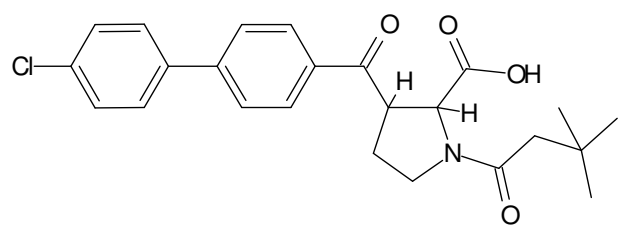

M1_9

MMP3 $\mathrm{Ha}$

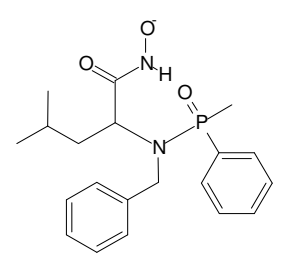

1b3d_lig

MMP3 PDB

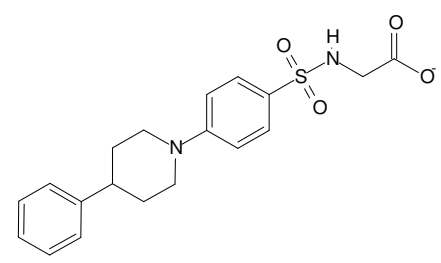

1b8y_lig

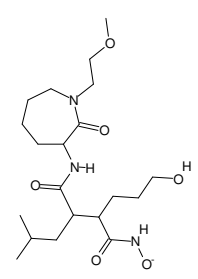

1biw_lig

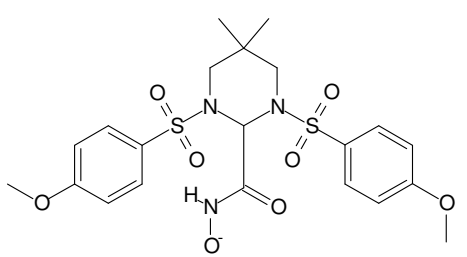

1bqo_lig

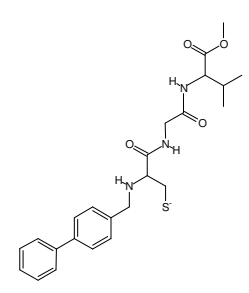

1c3i_lig

MMP3 PDB 
1ciz_lig

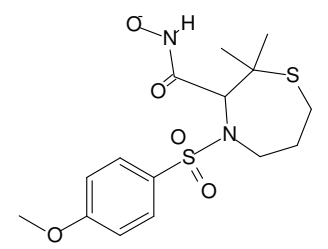

1d5j_lig

1d7x_lig

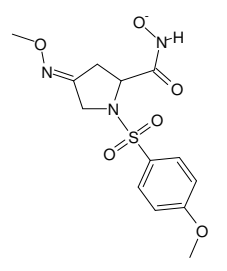

1d8f_lig
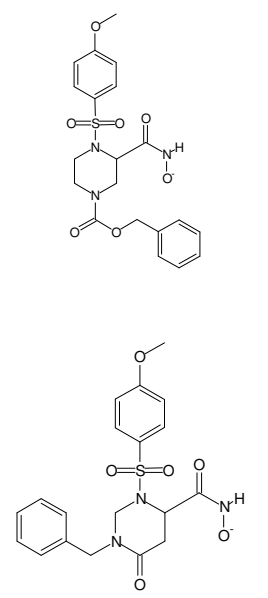

1d8m_lig

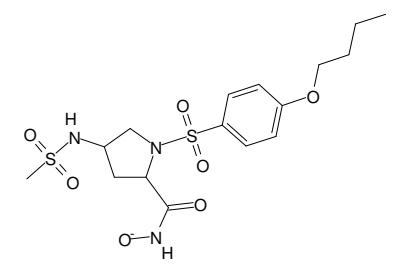

1g49_lig

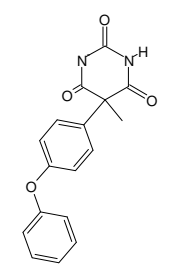

1g4k_lig

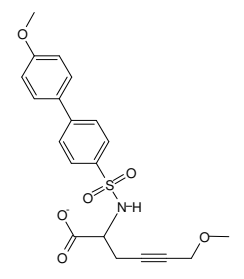

1hy7_lig
MMP3 PDB

MMP3 PDB

MMP3 PDB

MMP3 PDB

MMP3 PDB

MMP3 PDB

MMP3 PDB

MMP3 PDB 


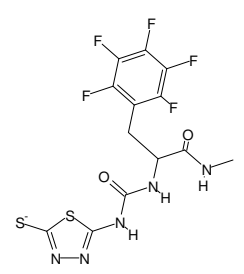

1usn_lig

MMP3 PDB

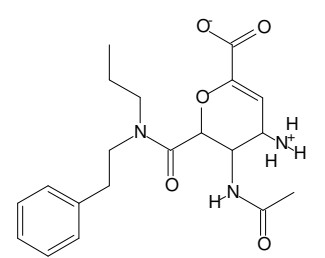

1bji_lig

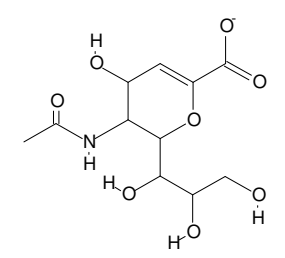

1f8b_lig

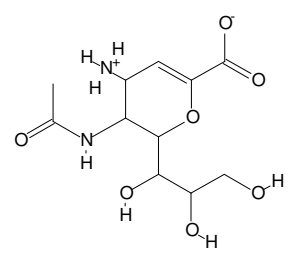

1f8c_lig

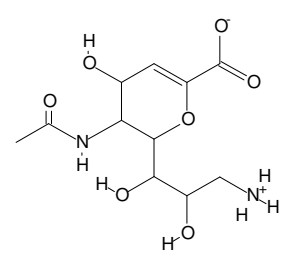

1f8d_lig

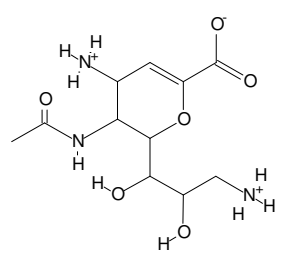

1f8e_lig

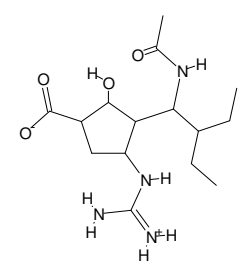

1I7f_lig

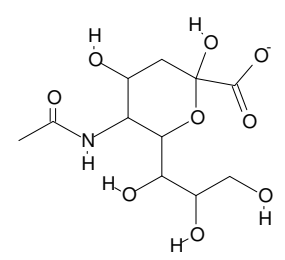

1mwe_lig

NA PDB 


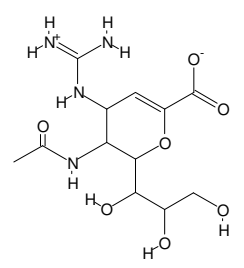

1nnc_lig

NA PDB

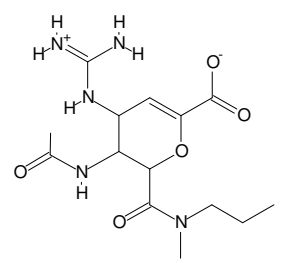

2qwi_lig

NA PDB

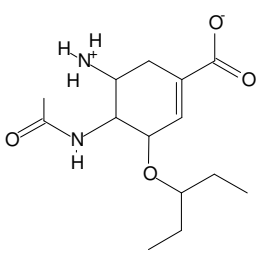

2qwk_lig

NA PDB

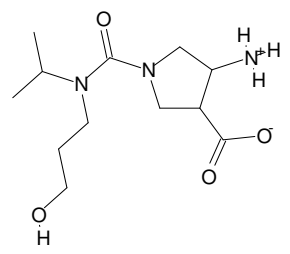

N10

NA Yi

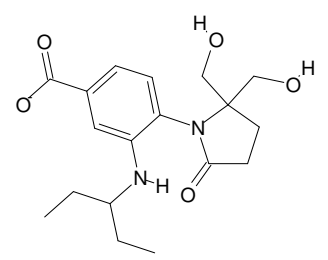

N11

NA Yi

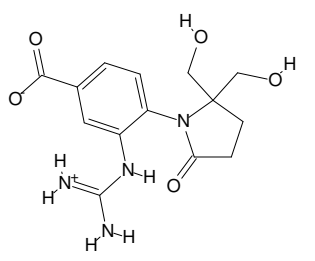

N12

NA Yi

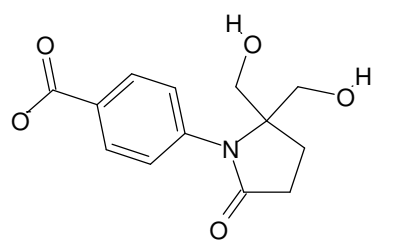

N13

NA Yi

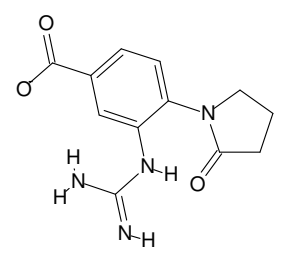




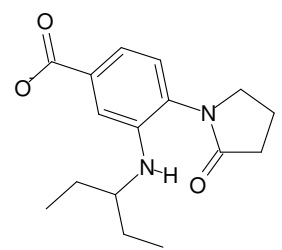

N15

NA $\quad \mathrm{Yi}$

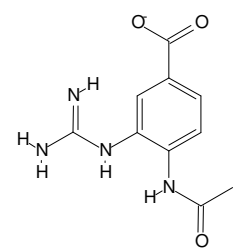

N16

NA Yi

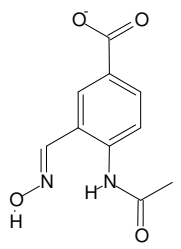

N17

NA Yi

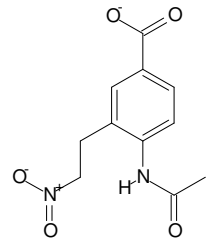

N18

NA $\quad$ Yi

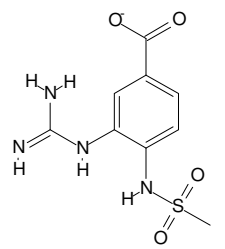

N19

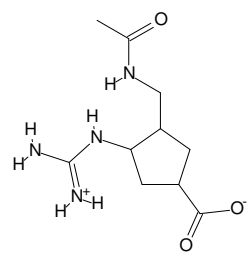

N2

NA Yi

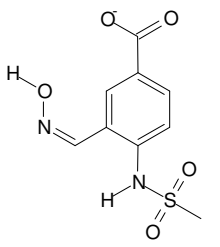

N20

NA $\quad \mathrm{Yi}$

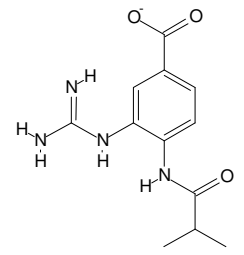




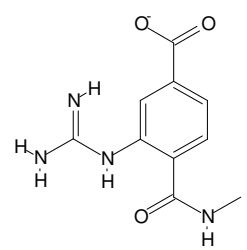

N22

NA Yi

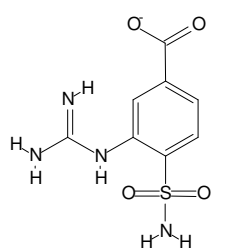

N23

NA $\quad Y i$

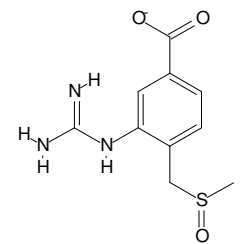

N24

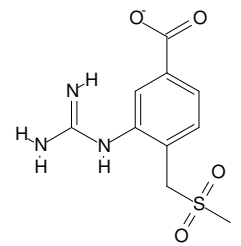

N25

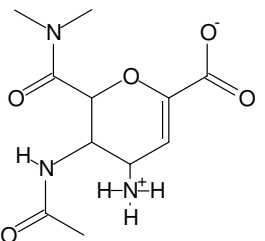

N26

NA $\quad Y i$

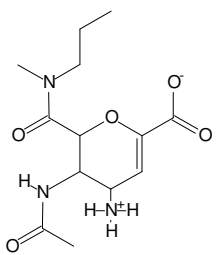

N27

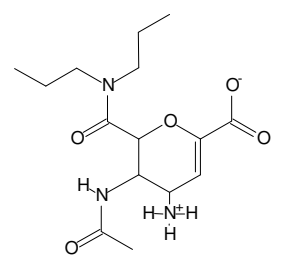

N28

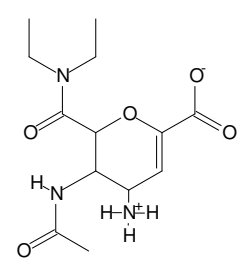




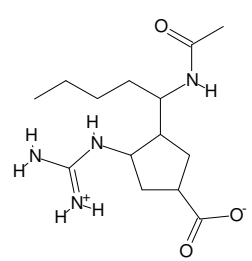

N3

NA $\quad \mathrm{Yi}$

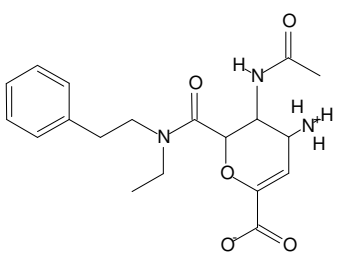

N30

NA Yi

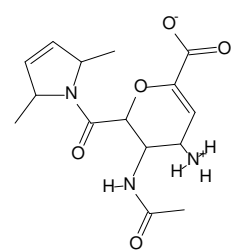

N31

NA $\quad \mathrm{Yi}$

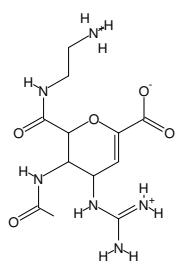

N32

NA $\quad$ Yi

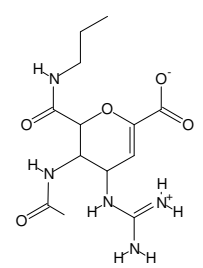

N33

NA Yi

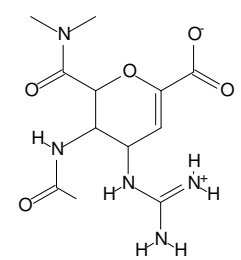

N34

NA Yi

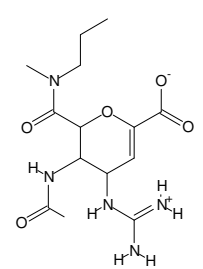

N35

NA Yi

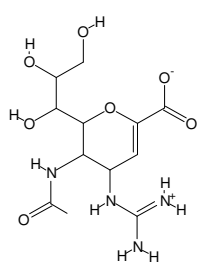




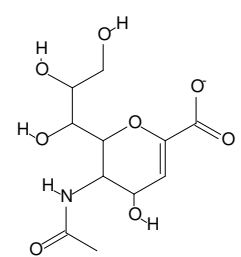

N37

NA $\quad$ Yi

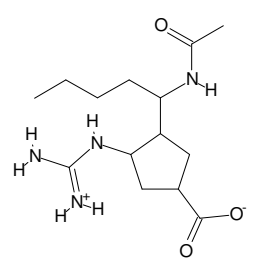

N4

NA $\quad \mathrm{Yi}$

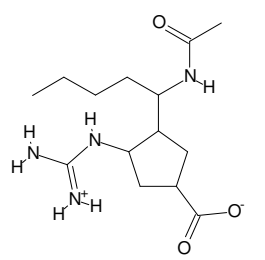

N5

NA $\quad \mathrm{Yi}$

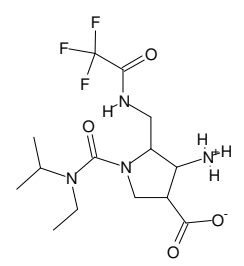

N6

NA $\quad$ Yi

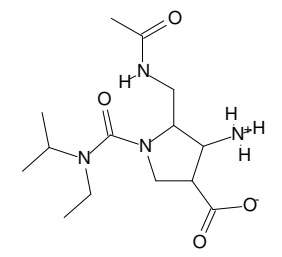

N7

NA Yi

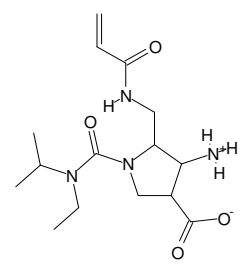

N8

NA Yi

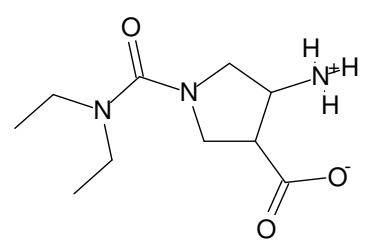

N9

NA $\quad$ Yi

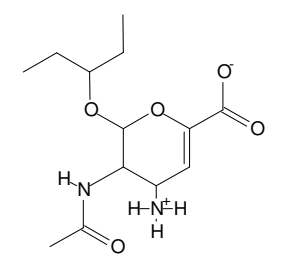




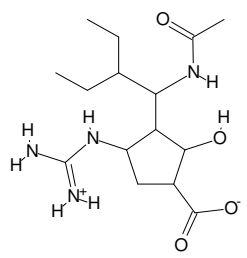

Peramivir

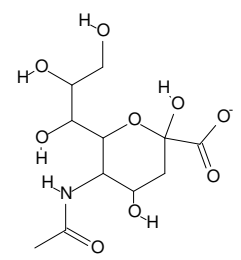

sialic_acid

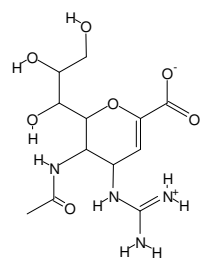

NA

NA

NA
Yi

Yi

Yi 